\title{
QUANTIZATION OF POISSON GROUPS
}

\author{
FABIO GAVARINI
}

Let $G^{\tau}$ be a connected simply connected semisimple algebraic group, endowed with generalized Sklyanin-Drinfel'd structure of Poisson group; let $\boldsymbol{H}^{\tau}$ be its dual Poisson group. By means of quantum double construction and dualization via formal Hopf algebras, we construct new quantum groups $U_{q, \varphi}^{M}(\mathfrak{h})$ - dual of $U_{q, \varphi}^{M^{\prime}}(\mathfrak{g})$ - which yield infinitesimal quantization of $H^{\tau}$ and $G^{\tau}$; we study their specializations at roots of 1 (in particular, their classical limits), thus discovering new quantum Frobenius morphisms. The whole description dualize for $H^{\tau}$ what was known for $G^{\tau}$, completing the quantization of the pair $\left(G^{\tau}, H^{\tau}\right)$.

\section{Introduction.}

\section{"Dualitas dualitatum et omnia dualitas" \\ N. Barbecue, "Scholia"}

Let $G$ be a semisimple, connected, and simply connected affine algebraic group over an algebraically closed field $k$ of characteristic zero; we consider a family of structures of Poisson group on $G$, indexed by a multiparameter $\tau$, which generalize the Sklyanin-Drinfel'd one. Then every such Poisson group $G^{\tau}$ has a dual Poisson group $H^{\tau}$, and $\mathfrak{g}^{\tau}:=\operatorname{Lie}\left(G^{\tau}\right)$ and $\mathfrak{h}^{\tau}:=\operatorname{Lie}\left(H^{\tau}\right)$ are Lie bialgebras dual of each other.

In 1985 Drinfel'd and Jimbo provided a quantization of $U(\mathfrak{g})=U\left(\mathfrak{g}^{0}\right)$, namely a Hopf algebra $U_{q}^{Q}(\mathfrak{g})$ over $k(q)$, presented by generators and relations, with a $k\left[q, q^{-1}\right]$-form $\mathfrak{U}^{Q}(\mathfrak{g})$ which for $q \rightarrow 1$ specializes to $U(\mathfrak{g})$ as a Poisson Hopf coalgebra. This has been extended to general parameter $\tau$ introducing multiparameter quantum groups $U_{q, \varphi}^{Q}(\mathfrak{g})$ (cf. $[\mathbf{R e}],[\mathbf{C V}-\mathbf{1}]$, $[\mathbf{C V}-2])$. Dually, one constructs a Hopf algebra $F_{q}^{P}[G]$ of matrix coefficients of $U_{q}^{Q}(\mathfrak{g})$ with a $k\left[q, q^{-1}\right]$-form $\mathfrak{F}^{P}[G]$ which specializes to $F[G]$, as a Poisson Hopf algebra, for $q \rightarrow 1$; in particular $\mathfrak{F}^{P}[G]$ is nothing but the Hopf subalgebra of "functions" in $F_{q}^{P}[G]$ which take values in $k\left[q, q^{-1}\right]$ when "evaluated" on $\mathfrak{U}^{Q}(\mathfrak{g})$ (in a word, the $k\left[q, q^{-1}\right]$-integer valued functions on $\left.\mathfrak{U}^{Q}(\mathfrak{g})\right)$. This again extends to general $\tau$ (cf. $\left.[\mathbf{C V}-\mathbf{2}]\right)$. 
So far the quantization only dealt with the Poisson group $G$ (or $G^{\tau}$ ); the dual group $H$ is involved defining a different $k\left[q, q^{-1}\right]$-form $\mathcal{U}^{P}(\mathfrak{g})$ (of a quantum group $U_{q}^{P}(\mathfrak{g})$ ) which specializes to $F[H]$ (as a Poisson Hopf algebra) for $q \rightarrow 1$ (cf. [DP]), with generalization to the multiparameter case possible again. Here sort of a "mixing dualities" (Hopf duality - among enveloping and function algebra - and Poisson duality - among dual Poisson groups) occurs, which was described (in a formal setting) by Drinfel'd (cf. $[\mathbf{D r}], \S 7$ ), and by Etingof and Kazhdan (cf. [EK-1], [EK-2]). This leads to consider the following: Let $F_{q}^{Q}[G]$ be the quantum function algebra dual of $U_{q}^{P}(\mathfrak{g})$, and look at the "dual" of $\mathcal{U}^{P}(\mathfrak{g})$ within $F_{q}^{Q}[G]$, call it $\mathcal{F}^{Q}[G]$, namely the Hopf algebra of $k\left[q, q^{-1}\right]$-integer valued functions on $\mathcal{U}^{P}(\mathfrak{g})$; then this should specialize to $U(\mathfrak{h}$ ) (as a Poisson Hopf coalgebra) for $q \rightarrow 1$; the same conjecture can be formulated in the multiparameter case too.

Our starting aim was to achieve this goal, i.e., to construct $F_{q}^{Q}[G]$ and its $k\left[q, q^{-1}\right]$-form $\mathcal{F}^{Q}[G]$, and to prove that $\mathcal{F}^{Q}[G]$ is a deformation of the Poisson Hopf coalgebra $U(\mathfrak{h})$. This goal is succesfully attained by performing a suitable dualization of the quantum double construction; but by the way, this leads to discover a new quantum group, which we call $U_{q}^{M}(\mathfrak{h})$, which is for $U(\mathfrak{h})$ what $U_{q}^{M}(\mathfrak{g})$ is for $U(\mathfrak{g})$; in particular it has an integer form $\mathfrak{U}^{Q}(\mathfrak{h})$ which is a quantization of $U(\mathfrak{h})$, and an integer form $\mathcal{U}^{P}(\mathfrak{h})$ which is a quantization of $F^{\infty}[G]$ (the function algebra of the formal Poisson group associated to $G$ ). Furthermore, we exhibit a Hopf pairing between $U_{q}^{M^{\prime}}(\mathfrak{g})$ and $U_{q}^{M}(\mathfrak{h})$ which gives a quantization of the various pairings occurring among the algebras attached to the pair $(G, H)$. Once again all this extends to the multiparameter case. Thus in particular we provide a (infinitesimal) quantization for a wide class of Poisson groups (the $H^{\tau}$ 's); now, in the summer of 1995 (when the present work was already accomplished) a quantization of any Poisson group was presented in [EK-1] and [EK-2]; but greatest generality implies lack of concreteness: in contrast, our construction is extremely concrete; moreover, it allows specialization at roots of 1 , construction of quantum Frobenius morphisms, and so on (like for $\mathfrak{U}^{Q}(\mathfrak{g})$ and $\left.\mathcal{U}^{P}(\mathfrak{g})\right)$, which is not possible in the approach of $[\mathbf{E K - 1}],[\mathbf{E K}-\mathbf{2}]$.

Finally, a brief sketch of the main ideas of the paper. Our aim is to study the "dual" of a quantum group $U_{q, \varphi}^{M}(\mathfrak{g})$ ( $M$ being a lattice of weights). First, we select as operation of "dualization" the most naïve one, namely taking the full linear dual (rather than the usual - restricted - Hopf dual), the latter being a formal Hopf algebra (rather than a common Hopf algebra). Second, as $U_{q, \varphi}^{M}(\mathfrak{g})$ is a quotient of a quantum double $D_{q, \varphi}^{M}(\mathfrak{g}):=$ $D\left(U_{q, \varphi}^{M}\left(\mathfrak{b}_{-}\right), U_{q, \varphi}^{M}\left(\mathfrak{b}_{+}\right), \pi_{\varphi}\right)$, its linear dual $U_{q, \varphi}^{M}(\mathfrak{g})^{*}$ embeds into $D_{q, \varphi}^{M}(\mathfrak{g})^{*}$. Third, since $D_{q, \varphi}^{M}(\mathfrak{g}) \cong U_{q, \varphi}^{M}\left(\mathfrak{b}_{+}\right) \otimes U_{q, \varphi}^{M}\left(\mathfrak{b}_{-}\right) \quad$ (as coalgebras) we have $D_{q, \varphi}^{M}(\mathfrak{g})^{*} \cong U_{q, \varphi}^{M}\left(\mathfrak{b}_{+}\right)^{*} \widehat{\otimes} U_{q, \varphi}^{M}\left(\mathfrak{b}_{-}\right)^{*}$ (as algebras), where $\widehat{\otimes}$ denotes topological tensor product. Fourth, since quantum Borel algebras of opposite sign 
are perfectly paired their linear duals are suitable completions of quantum Borel algebras again: Thus we find a presentation of $U_{q, \varphi}^{M}(\mathfrak{g})^{*}$ by generators and relations which leads us to define $U_{q, \varphi}^{M}(\mathfrak{h}):=U_{q, \varphi}^{M^{\prime}}(\mathfrak{g}){ }^{*}$ (where $M^{\prime}$ depends on $M$ ) and gives us all claimed results; because of their construction we call the new objects $U_{q, \varphi}^{M}(\mathfrak{h})$ (multiparameter) quantum formal groups.

In contrast, we also present an alternative approach, yielding other new objects - denoted by $F_{q, \varphi}^{M, \infty}[G]$ - which we call (multiparameter) formal quantum groups; the similar but different terminology reveals the fact that $U_{q, \varphi}^{M}(\mathfrak{h})$ and $F_{q, \varphi}^{M, \infty}[G]$ provide two different quantizations of the same classical objects $U\left(\mathfrak{h}^{\tau}\right)$ and $F^{\infty}\left[G^{\tau}\right]$, arising from two different ways of realizing $F^{\infty}\left[G^{\tau}\right]$.

Acknowledgements. The author wishes to thank C. Procesi for several fruitful conversations, and C. De Concini for some useful talk; he is also endebted with M. Costantini, M. Varagnolo, and I. Damiani for many helpful discussions.

\section{The classical objects.}

1.1. Cartan data. Let $A:=\left(a_{i j}\right)_{i, j=1, \ldots, n}$ be a $n \times n$ symmetrizable Cartan matrix; thus $a_{i j} \in \mathbb{Z}, a_{i i}=2, a_{i j} \leq 0$ if $i \neq j$, and there exists a vector $\left(d_{1}, \ldots, d_{n}\right)$ with relatively prime positive integral entries $d_{i}$ such that $\left(d_{i} a_{i j}\right)_{i, j=1, \ldots, n}$; is a symmetric positive definite matrix. Define the weight lattice $P$ to be the lattice with basis $\left\{\omega_{1}, \ldots, \omega_{n}\right\}$; let $P_{+}:=\sum_{i=1}^{n} \mathbb{N} \omega_{i}$ be the subset of dominant integral weights, $\alpha_{j}:=\sum_{i=1}^{n} a_{i j} \omega_{i}(j=1, \ldots, n)$ the simple roots, $Q:=\sum_{j=1}^{n} \mathbb{Z} \alpha_{j}(\subset P)$ the root lattice, and $Q_{+}:=\sum_{j=1}^{n} \mathbb{N} \alpha_{j}$ the positive root lattice. Let $W$ be the Weyl group associated to $A$, and let $\Pi:=\left\{\alpha_{1}, \ldots, \alpha_{n}\right\}$ : Then $R:=W(\Pi)$ is the set of roots, $R^{+}=R \cap Q_{+}$ the set of positive roots; finally, we set $N:=\#\left(R^{+}\right)(=|W|)$. Define bilinear pairings $\langle\mid\rangle: Q \times P \rightarrow \mathbb{Z}$ and $(\mid): Q \times P \rightarrow \mathbb{Z}$ by $\left\langle\alpha_{i} \mid \omega_{j}\right\rangle=\delta_{i j}$ and $\left(\alpha_{i} \mid \omega_{j}\right)=\delta_{i j} d_{i}$. Then $\left(\alpha_{i} \mid \alpha_{j}\right)=d_{i} a_{i j}$, giving a symmetric $\mathbb{Z}$-valued $W$-invariant bilinear form on $Q$ such that $(\alpha \mid \alpha) \in 2 \mathbb{Z}$. For all $\alpha \in R^{+}$, let $d_{\alpha}:=\frac{(\alpha \mid \alpha)}{2}$; then $d_{\alpha_{i}}=d_{i}$ for all $i=1, \ldots, n$. We also extend the $(\mid): Q \times P \rightarrow \mathbb{Z}$ to a (non-degenerate) pairing $(\mid): \mathbb{Q} Q \times \mathbb{Q} P \rightarrow \mathbb{Q}$ of $\mathbb{Q}$-vector spaces by scalar extension, where $\mathbb{Q} T:=\mathbb{Q} \otimes_{\mathbb{Z}} T(T=Q, P)$ : Then restriction gives a pairing $(\mid): P \times P \rightarrow \mathbb{Q}$ (looking at $P$ as a sublattice of $\mathbb{Q} Q)$, which takes values in $\mathbb{Z}\left[D^{-1}\right]$, where $D:=\operatorname{det}\left(\left(a_{i j}\right)_{i, j=1}^{n}\right)$. Given any pair of lattices $\left(M, M^{\prime}\right)$, with $Q \leq M, M^{\prime} \leq \mathbb{Q} P$, we say that they are dual of each other if $M^{\prime}=\{y \in \mathbb{Q} P \mid\langle M, y\rangle \subseteq \mathbb{Z}\}, M=\{x \in$ $\left.\mathbb{Q} P \mid\left\langle x, M^{\prime}\right\rangle \subseteq \mathbb{Z}\right\}$, the two conditions being equivalent; then for any lattice $M$ with $Q \leq M \leq P$ there exists a unique dual lattice $M^{\prime}$ such that $Q \leq M^{\prime} \leq P$ and $(\mid): \mathbb{Q} P \times \mathbb{Q} P \rightarrow \mathbb{Q}$ restricts to a perfect pairing 
$(\mid): M \times M^{\prime} \rightarrow \mathbb{Z}$; in particular $P^{\prime}=Q$ and $Q^{\prime}=P$. In the sequel we denote by $\left\{\mu_{1}, \ldots, \mu_{n}\right\}$ and $\left\{\nu_{1}, \ldots, \nu_{n}\right\}$ fixed $\mathbb{Z}$-bases of $M$ and $M^{\prime}$ dual of each other, i.e., such that $\left(\mu_{i} \mid \nu_{j}\right)=\delta_{i j}$ for all $i, j=1, \ldots, n$, and we set $M_{+}:=M \cap P_{+}$.

In the following our constructions will work in general for the pairs of dual lattices $(P, Q)$ and $(Q, P)$; but in the simply laced case (in which $\langle\rangle=$, $()),\left(M, M^{\prime}\right)$ will be any pair of dual lattices.

1.2. The Poisson groups $G$ and $H$. Let $G$ be a connected simplyconnected semisimple affine algebraic group over an algebraically closed field $k$ of characteristic 0. Fix a maximal torus $T \leq G$ and opposite Borel subgroups $B_{ \pm}$, with unipotent subgroups $U_{ \pm}$, such that $B_{+} \cap B_{-}=T$, and let $\mathfrak{g}:=\operatorname{Lie}(G), \mathfrak{t}:=\operatorname{Lie}(T), \mathfrak{b}_{ \pm}:=\operatorname{Lie}\left(B_{ \pm}\right), \mathfrak{n}_{ \pm}:=\operatorname{Lie}\left(U_{ \pm}\right) ;$fix also $\tau:=\left(\tau_{1}, \ldots, \tau_{n}\right) \in Q^{n}$ such that $\left(\tau_{i}, \alpha_{j}\right)=-\left(\tau_{j}, \alpha_{i}\right)$ for all $i, j=1, \ldots, n$ : When $\tau=(0, \ldots, 0)$ we shall simply skip it throughout. Set $K=G \times G$, define $G^{\tau}:=G$ embedded in $K$ as the diagonal subgroup, and define a second subgroup

$$
\begin{aligned}
H^{\tau}:=\left\{\left(u_{-} t_{-}, t_{+} u_{+}\right) \mid u_{ \pm} \in U_{ \pm}, t_{ \pm} \in T, t_{-} t_{+} \in \exp \left(\mathfrak{t}^{\tau}\right)\right\} & \\
& \quad\left(\leq B_{-} \times B_{+} \leq K\right)
\end{aligned}
$$

where $\mathfrak{t}^{\tau}:=\sum_{i=1}^{n} k \cdot h_{-\alpha_{i}+2 \tau_{i}} \oplus h_{\alpha_{i}+2 \tau_{i}} \leq \mathfrak{t} \oplus \mathfrak{t} \leq \mathfrak{g} \oplus \mathfrak{g}=\mathfrak{k}:=\operatorname{Lie}(K)$; hence we have $\mathfrak{h}^{\tau}:=\operatorname{Lie}\left(H^{\tau}\right)=\left(\mathfrak{n}_{-}, 0\right) \oplus \mathfrak{t}^{\tau} \oplus\left(0, \mathfrak{n}_{+}\right)$. The triple $\left(K, G^{\tau}, H^{\tau}\right)$ is an algebraic Manin triple (cf. [DP], §11), whose invariant form is defined as follows: First normalize the Killing form ( , ) on $\mathfrak{g}$ so that short roots have square length 2 ; then define the form on $\mathfrak{k}=\mathfrak{g} \oplus \mathfrak{g}$ by ${ }^{1}$

$$
\left\langle x_{1} \oplus y_{1}, x_{2} \oplus y_{2}\right\rangle:=\frac{1}{2}\left(y_{1}, y_{2}\right)-\frac{1}{2}\left(x_{1}, x_{2}\right) .
$$

In general, if $\left(\mathfrak{k}^{\prime}, \mathfrak{g}^{\prime}, \mathfrak{h}^{\prime}\right)$ is any Manin triple, the bilinear form on $\mathfrak{k}^{\prime}$ gives by restriction a non-degenerate pairing $\langle\rangle:, \mathfrak{h}^{\prime} \otimes \mathfrak{g}^{\prime} \rightarrow k$ which is a pairing of Lie bialgebras, that is

$$
\left\langle x,\left[y_{1}, y_{2}\right]\right\rangle=\left\langle\delta(x), y_{1} \otimes y_{2}\right\rangle, \quad\left\langle\left[x_{1}, x_{2}\right], y\right\rangle=\left\langle x_{1} \otimes x_{2}, \delta(y)\right\rangle
$$

where $\delta$ is the Lie cobracket; we shall call it Poisson pairing. In the present case we denote it by $\pi_{\mathcal{P}}^{\tau}(h, g):=\langle h, g\rangle$; it is described by

$$
\begin{array}{ccc}
\left\langle\mathrm{f}_{i}^{\tau}, f_{j}\right\rangle=0 & \left\langle\mathrm{f}_{i}^{\tau}, h_{j}\right\rangle=0 & \left\langle\mathrm{f}_{i}^{\tau}, e_{j}\right\rangle=-\frac{1}{2} \delta_{i j} d_{i}^{-1} \\
\left\langle\mathrm{~h}_{i}^{\tau}, f_{j}\right\rangle=0 & \left\langle\mathrm{~h}_{i}^{\tau}, h_{j}\right\rangle=a_{i j} d_{j}^{-1}=a_{j i} d_{i}^{-1} & \left\langle\mathrm{~h}_{i}^{\tau}, e_{j}\right\rangle=0 \\
\left\langle\mathrm{e}_{i}^{\tau}, f_{j}\right\rangle=\frac{1}{2} \delta_{i j} d_{i}^{-1} & \left\langle\mathrm{e}_{i}^{\tau}, h_{j}\right\rangle=0 & \left\langle\mathrm{e}_{i}^{\tau}, e_{j}\right\rangle=0
\end{array}
$$

where the $\mathrm{f}_{s}^{\tau}, \mathrm{h}_{s}^{\tau}, \mathrm{e}_{s}^{\tau}$, resp. $f_{s}, h_{s}, e_{s}$, are Chevalley-type generators of $\mathfrak{h}^{\tau}$, resp. $\mathfrak{g}^{\tau}$, embedded inside $\mathfrak{k}=\mathfrak{g} \oplus \mathfrak{g}$, namely $\mathrm{f}_{s}^{\tau}=f_{s} \oplus 0, \mathrm{~h}_{s}^{\tau}=$

\footnotetext{
${ }^{1}$ Warning: Beware of the normalization of the invariant form of $\mathfrak{k}$, which is different from $[\mathbf{D P}]$.
} 
$h_{-\alpha_{s}+2 \tau_{s}} \oplus h_{\alpha_{s}+2 \tau_{s}}, \mathrm{e}_{s}^{\tau}=0 \oplus e_{s}$, and $f_{s}=f_{s} \oplus f_{s}, h_{s}=h_{s} \oplus h_{s}, e_{s}=e_{s} \oplus e_{s}$ (see $\S \S 1.3-4$ below).

1.3. The Poisson Hopf coalgebra $U\left(\mathfrak{g}^{\tau}\right)$. The universal enveloping algebra $U\left(\mathfrak{g}^{\tau}\right)=U(\mathfrak{g})$ can be presented as the associative $k$-algebra with 1 generated by elements $, f_{i}, h_{i}, e_{i}(i=1, \ldots, n)$ (the Chevalley generators) satisfying Serre's relations; it has a canonical structure of Hopf algebra, given by $\Delta(x)=x \otimes 1+1 \otimes x, S(x)=-x, \epsilon(x)=0$ for $x=f_{i}, h_{i}, e_{i}$; finally, the Lie cobracket $\delta=\delta_{\mathfrak{g}^{\tau}}: \mathfrak{g}^{\tau} \longrightarrow \mathfrak{g}^{\tau} \otimes \mathfrak{g}^{\tau}$ extends to a Poisson cobracket $\delta: U\left(\mathfrak{g}^{\tau}\right) \longrightarrow U\left(\mathfrak{g}^{\tau}\right) \otimes U\left(\mathfrak{g}^{\tau}\right)$ (compatible with the Hopf structure) given by $\delta\left(f_{i}\right)=\frac{\left(\alpha_{i}+2 \tau_{i} \mid \alpha_{i}+2 \tau_{i}\right)}{2} h_{\alpha_{i}+2 \tau_{i}} \otimes f_{i}-\frac{\left(\alpha_{i}+2 \tau_{i} \mid \alpha_{i}+2 \tau_{i}\right)}{2} f_{i} \otimes h_{\alpha_{i}+2 \tau_{i}}, \quad \delta\left(h_{i}\right)=0$, $\delta\left(e_{i}\right)=\frac{\left(\alpha_{i}-2 \tau_{i} \mid \alpha_{i}-2 \tau_{i}\right)}{2} h_{\alpha_{i}-2 \tau_{i}} \otimes e_{i}-\frac{\left(\alpha_{i}-2 \tau_{i} \mid \alpha_{i}-2 \tau_{i}\right)}{2} e_{i} \otimes h_{\alpha_{i}-2 \tau_{i}}$.

1.4. The Poisson Hopf coalgebra $U\left(\mathfrak{h}^{\tau}\right)$. From the very definition and the previous presentation of $U\left(\mathfrak{g}^{\tau}\right)$ we get for $U\left(\mathfrak{h}^{\tau}\right)$ the following presentation. $U\left(\mathfrak{h}^{\tau}\right)$ is the associative $k$-algebra with 1 generated by $\mathrm{f}_{i}^{\tau}, \mathrm{h}_{i}^{\tau}, \mathrm{e}_{i}^{\tau}$ $(i=1, \ldots, n)$ with relations

$$
\begin{gathered}
\mathrm{h}_{i}^{\tau} \mathrm{h}_{j}^{\tau}-\mathrm{h}_{j}^{\tau} \mathrm{h}_{i}^{\tau}=0, \quad \mathrm{e}_{i}^{\tau} \mathrm{f}_{j}^{\tau}-\mathrm{f}_{j}^{\tau} \mathrm{e}_{i}^{\tau}=0 \\
\mathrm{~h}_{i}^{\tau} \mathrm{f}_{j}^{\tau}-\mathrm{f}_{j}^{\tau} \mathrm{h}_{i}^{\tau}=\left\langle\alpha_{i}-2 \tau_{i}, \alpha_{j}\right\rangle \mathrm{f}_{j}^{\tau}, \quad \mathrm{h}_{i}^{\tau} \mathrm{e}_{j}^{\tau}-\mathrm{e}_{j}^{\tau} \mathrm{h}_{i}^{\tau}=\left\langle\alpha_{i}+2 \tau_{i}, \alpha_{j}\right\rangle \mathrm{e}_{j}^{\tau} \\
\sum_{k=0}^{1-a_{i j}}(-1)^{k}\left(\begin{array}{c}
1-a_{i j} \\
k
\end{array}\right)\left(\mathrm{f}_{i}^{\tau}\right)^{1-a_{i j}-k} \mathrm{f}_{j}^{\tau}\left(\mathrm{f}_{i}^{\tau}\right)^{k}=0 \quad(i \neq j) \\
\sum_{k=0}^{1-a_{i j}}(-1)^{k}\left(\begin{array}{c}
1-a_{i j} \\
k
\end{array}\right)\left(\mathrm{e}_{i}^{\tau}\right)^{1-a_{i j}-k} \mathrm{e}_{j}^{\tau}\left(\mathrm{e}_{i}^{\tau}\right)^{k}=0 \quad(i \neq j)
\end{gathered}
$$

for all $i, j=1, \ldots, n$; its natural Hopf structure is given by

$$
\Delta(x)=x \otimes 1+1 \otimes x, \quad S(x)=-x, \quad \epsilon(x)=0
$$

for $x=\mathrm{f}_{i}^{\tau}, \mathrm{h}_{i}^{\tau}, \mathrm{e}_{i}^{\tau}$, and the co-Poisson structure $\delta=\delta_{\mathfrak{h}^{\tau}}: U\left(\mathfrak{h}^{\tau}\right) \longrightarrow U\left(\mathfrak{h}^{\tau}\right) \otimes$ $U\left(\mathfrak{h}^{\tau}\right)$ by

$$
\begin{aligned}
\delta\left(\mathrm{f}_{i}^{\tau}\right)= & d_{i} \cdot\left(\mathrm{h}_{i}^{\tau} \otimes \mathrm{f}_{i}^{\tau}-\mathrm{f}_{i}^{\tau} \otimes \mathrm{h}_{i}^{\tau}\right) \\
& +2 d_{i}^{-1} \cdot \sum_{\alpha, \beta \in R^{+}} c_{\alpha, \beta}^{i,+} d_{\alpha} d_{\beta} \cdot\left(\mathrm{e}_{\alpha}^{\tau} \otimes \mathrm{f}_{\beta}^{\tau}-\mathrm{f}_{\beta}^{\tau} \otimes \mathrm{e}_{\alpha}^{\tau}\right) \\
\delta\left(\mathrm{h}_{i}^{\tau}\right)= & 4 d_{i}^{-1} \cdot \sum_{\gamma \in R^{+}} d_{\gamma}\left(\gamma \mid \alpha_{i}\right) \cdot\left(\mathrm{e}_{\gamma}^{\tau} \otimes \mathrm{f}_{\gamma}^{\tau}-\mathrm{f}_{\gamma}^{\tau} \otimes \mathrm{e}_{\gamma}^{\tau}\right) \\
\delta\left(\mathrm{e}_{i}^{\tau}\right)= & d_{i} \cdot\left(\mathrm{e}_{i}^{\tau} \otimes \mathrm{h}_{i}^{\tau}-\mathrm{h}_{i}^{\tau} \otimes \mathrm{e}_{i}^{\tau}\right) \\
& +2 d_{i}^{-1} \cdot \sum_{\alpha, \beta \in R^{+}} c_{\alpha, \beta}^{i,-} d_{\alpha} d_{\beta} \cdot\left(\mathrm{f}_{\beta}^{\tau} \otimes \mathrm{e}_{\alpha}^{\tau}-\mathrm{e}_{\alpha}^{\tau} \otimes \mathrm{f}_{\beta}^{\tau}\right)
\end{aligned}
$$

with the $\mathrm{e}_{\gamma}^{\tau}$ 's and the $\mathrm{f}_{\gamma}^{\tau}$ 's given by $\left\langle\mathrm{e}_{\gamma}^{\tau}, f_{\eta}\right\rangle=+\delta_{\gamma, \eta} d_{\gamma} / 2,\left\langle\mathrm{e}_{\gamma}^{\tau}, h_{i}\right\rangle=0$, $\left\langle\mathrm{e}_{\gamma}^{\tau}, e_{\eta}\right\rangle=0$, and $\left\langle\mathrm{f}_{\gamma}^{\tau}, f_{\eta}\right\rangle=0,\left\langle\mathrm{f}_{\gamma}^{\tau}, h_{i}\right\rangle=0,\left\langle\mathrm{f}_{\gamma}^{\tau}, e_{\eta}\right\rangle=-\delta_{\gamma, \eta} d_{\gamma} / 2\left(f_{\eta}\right.$ and 
$e_{\eta}$ being root vectors in $\left.\mathfrak{g}^{\tau}\right)$, and the $c_{\alpha, \beta}^{i, \pm}$, s given by $\left[f_{\alpha}, e_{\beta}\right]=c_{\alpha, \beta}^{i,-} \cdot f_{i}$, $\left[f_{\alpha}, e_{\beta}\right]=c_{\alpha, \beta}^{i,+} \cdot e_{i}$.

\section{Quantum Borel algebras and DRT pairings.}

2.1. Notations. For all $s, n \in \mathbb{N}$, let $(n)_{q}:=\frac{q^{n}-1}{q-1}(\in k[q]),(n)_{q} !:=$ $\prod_{r=1}^{n}(r)_{q},\left(\begin{array}{l}n \\ s\end{array}\right)_{q}:=\frac{(n)_{q} !}{(s)_{q} !(n-s)_{q} !}(\in k[q])$, and $[n]_{q}:=\frac{q^{n}-q^{-n}}{q-q^{-1}}\left(\in k\left[q, q^{-1}\right]\right)$, $[n]_{q} !:=\prod_{r=1}^{n}[r]_{q},\left[\begin{array}{l}n \\ s\end{array}\right]_{q}:=:=\frac{[n]_{q} !}{[s]_{q} ![n-s]_{q} !}\left(\in k\left[q, q^{-1}\right]\right)$; let $q_{\alpha}:=q^{d_{\alpha}}$ for all $\alpha \in R^{+}$, and $q_{i}:=q_{\alpha_{i}}$. Let $Q, P$ be as in $\S 1$; we fix an endomorphism $\varphi$ of the $\mathbb{Q}$-vector space $\mathbb{Q} P:=\mathbb{Q} \otimes_{\mathbb{Z}} P$ which is antisymmetric — with respect to $(\mid)$ - and satisfies the conditions

$$
\varphi(Q) \subseteq Q, \quad \frac{1}{2}(\varphi(P) \mid P) \subseteq \mathbb{Z}, \quad 2 A Y A^{-1} \in \operatorname{Mat}_{n}(\mathbb{Z})
$$

where, letting $\tau_{i}:=\frac{1}{2} \varphi\left(\alpha_{i}\right)=\sum_{j=1}^{n} y_{j i} \alpha_{j}$, we set $Y:=\left(y_{i j}\right)_{i, j=1, \ldots, n ;}$. We also define $\tau_{\alpha}:=\frac{1}{2} \varphi(\alpha)$ for all $\alpha \in R$ (so $\tau_{\alpha_{i}}=\tau_{i}$ ). It is proved in $[\mathbf{C V}-1]$ that $\left(\operatorname{id}_{\mathbb{Q} P}+\varphi\right)$ and $\left(\operatorname{id}_{\mathbb{Q} P}-\varphi\right)$ are isomorphisms: Then we set $r:=\left(\operatorname{id}_{\mathbb{Q} P}+\varphi\right)^{-1}, \bar{r}:=\left(\operatorname{id}_{\mathbb{Q} P}-\varphi\right)^{-1}$.

2.2. Quantum Borel algebras. From now on $M$ will be any lattice such that $Q \leq M \leq P$; then $M^{\prime}$ will be the dual lattice defined in $\S 1.1$, according to the conditions therein. As in $[\mathbf{C V}-\mathbf{1}], U_{q, \varphi}^{M}\left(\mathfrak{b}_{-}\right)$, resp. $U_{q, \varphi}^{M}\left(\mathfrak{b}_{+}\right)$, is the associative $k(q)$-algebra with 1 generated by $L_{\mu}(\mu \in M), F_{1}, \ldots, F_{n}$, resp. $L_{\mu}(\mu \in M), E_{1}, \ldots, E_{n}$, with relations

$$
L_{0}=1, \quad L_{\mu} L_{\nu}=L_{\mu+\nu},
$$

$$
L_{\mu} F_{j}=q^{-\left(\alpha_{j} \mid \mu\right)} F_{j} L_{\mu}, \quad \sum_{p+s=1-a_{i j}}(-1)^{s}\left[\begin{array}{c}
1-a_{i j} \\
s
\end{array}\right]_{q_{i}} F_{i}^{p} F_{j} F_{i}^{s}=0
$$

resp. $\quad L_{\mu} E_{j}=q^{+\left(\alpha_{j} \mid \mu\right)} E_{j} L_{\mu}, \quad \sum_{p+s=1-a_{i j}}(-1)^{s}\left[\begin{array}{c}1-a_{i j} \\ s\end{array}\right]_{q_{i}} E_{i}^{p} E_{j} E_{i}^{s}=0$

for all $i, j=1, \ldots, n$ and $\mu, \nu \in M$; both are Hopf algebras, with

$$
\begin{array}{ccc}
\Delta_{\varphi}\left(F_{i}\right)=F_{i} \otimes L_{-\alpha_{i}-\tau_{i}}+L_{\tau_{i}} \otimes F_{i}, & \epsilon_{\varphi}\left(F_{i}\right)=0, & S_{\varphi}\left(F_{i}\right)=-F_{i} L_{\alpha_{i}} \\
\Delta_{\varphi}\left(L_{\mu}\right)=L_{\mu} \otimes L_{\mu}, & \epsilon_{\varphi}\left(L_{\mu}\right)=1, & S_{\varphi}\left(L_{\mu}\right)=L_{-\mu} \\
\Delta_{\varphi}\left(E_{i}\right)=E_{i} \otimes L_{\tau_{i}}+L_{\alpha_{i}-\tau_{i}} \otimes E_{i}, & \epsilon_{\varphi}\left(E_{i}\right)=0, & S_{\varphi}\left(E_{i}\right)=-L_{-\alpha_{i}} E_{i}
\end{array}
$$

for all $i=1, \ldots, n, \mu \in M$. We also consider the subalgebras $U_{q, \varphi}^{M}(\mathfrak{t})$ (generated by the $L_{\mu}$ 's), $U_{q, \varphi}\left(\mathfrak{n}_{-}\right)$(generated by the $F_{i}$ 's), $U_{q, \varphi}\left(\mathfrak{n}_{+}\right)$(generated by the $E_{i}$ 's). In the sequel we shall use the notation $K_{\alpha}:=L_{\alpha}$, $M_{\mu}:=L_{\mu}, \Lambda_{\nu}:=L_{\nu}\left(\forall \alpha \in Q, \mu \in M, \nu \in M^{\prime}\right)$ (and in particular $\left.K_{i}:=K_{\alpha_{i}}, M_{i}:=M_{\mu_{i}} \Lambda_{i}:=\Lambda_{\nu_{i}}\right)$, and $U_{\leq}^{M}:=U_{q, \varphi}^{M}\left(\mathfrak{b}_{-}\right), U_{\geq}^{M}:=U_{q, \varphi}^{M}\left(\mathfrak{b}_{+}\right)$, 
$U_{\varphi, 0}^{M}:=U_{q, \varphi}^{M}(\mathfrak{t}), U_{-}:=U_{q, \varphi}\left(\mathfrak{n}_{-}\right), U_{+}:=U_{q, \varphi}\left(\mathfrak{n}_{+}\right)$. If $\varphi=0$ we just skip it througout.

Finally, multiplication yields isomorphisms

$$
U_{\varphi, \leq}^{M} \cong U_{\varphi,-} \otimes U_{\varphi, 0}^{M} \cong U_{\varphi, 0}^{M} \otimes U_{\varphi,-}, \quad U_{\varphi, \geq}^{M} \cong U_{\varphi,+} \otimes U_{\varphi, 0}^{M} \cong U_{\varphi, 0}^{M} \otimes U_{\varphi,+} .
$$

2.3. DRT pairings. If $H$ is any Hopf algebra, we let $H^{o p}$ be the same coalgebra with opposite multiplication, and $H_{o p}$ the same algebra with opposite comultiplication.

From $[\mathbf{C V - 1}], \S 3$, there exist perfect (i.e., non-degenerate) pairings of Hopf algebras

$$
\begin{array}{rlrl}
\pi_{\varphi}:\left(U_{\varphi, \leq}^{M}\right)_{o p} \otimes U_{\varphi, \geq}^{M^{\prime}} \longrightarrow k(q), & \pi_{\varphi}: U_{\varphi, \leq}^{M} \otimes\left(U_{\varphi, \geq}^{M^{\prime}}\right)^{o p} \longrightarrow k(q) \\
\overline{\pi_{\varphi}}:\left(U_{\varphi, \geq}^{M}\right)_{o p} \otimes U_{\varphi, \leq}^{M^{\prime}} \longrightarrow k(q), & & \overline{\pi_{\varphi}}: U_{\varphi, \geq}^{M} \otimes\left(U_{\varphi, \leq}^{M^{\prime}}\right)^{o p} \longrightarrow k(q) \\
\pi_{\varphi}\left(L_{\mu}, L_{\nu}\right)=q^{-(r(\mu) \mid \nu)}, & \pi_{\varphi}\left(L_{\mu}, E_{j}\right)=0, \\
\pi_{\varphi}\left(F_{i}, L_{\nu}\right)=0, & \pi_{\varphi}\left(F_{i}, E_{j}\right)=\delta_{i j} \frac{q^{-\left(r\left(\tau_{i}\right) \mid \tau_{i}\right)}}{\left(q_{i}^{-1}-q_{i}\right)} \\
\pi_{\varphi}\left(L_{\mu}, L_{\nu}\right)=q^{+(r(\mu) \mid \nu)}, & \overline{\pi_{\varphi}}\left(E_{i}, L_{\nu}\right)=0, \\
\pi_{\varphi}\left(L_{\mu}, F_{j}\right)=0, & \overline{\pi_{\varphi}}\left(E_{i}, F_{j}\right)=\delta_{i j} \frac{q^{+\left(r\left(\tau_{i}\right) \mid \tau_{i}\right)}}{\left(q_{i}-q_{i}^{-1}\right)} .
\end{array}
$$

These pairings were introduced by Drinfel'd, Rosso, Tanisaki, and others, whence we shall call them DRT pairings. If $\pi$ is any DRT pairing we shall also set $\langle x, y\rangle_{\pi}$ for $\pi(x, y)$.

2.4. PBW bases. Let $N:=\#\left(R^{+}\right)$, and fix any total convex ordering (cf. [Pa] and [DP], §8.2) $\alpha^{1}, \alpha^{2}, \ldots, \alpha^{N}$ of $R^{+}$: Following Lusztig we can construct root vectors $E_{\alpha^{r}},(r=1, \ldots, N)$ as in $[\mathbf{D P}]$ or $[\mathbf{C V}-\mathbf{1}]$ and get PBW bases of increasing ordered monomials $\left\{L_{\mu} \cdot \prod_{r=1}^{N} F_{\alpha^{r}}^{f_{r}} \mid \mu \in M\right.$; $\left.f_{1}, \ldots, f_{N} \in \mathbb{N}\right\}$ for $U_{\varphi, \leq}^{M}$ and $\left\{L_{\mu} \cdot \prod_{r=1}^{N} E_{\alpha^{r}}^{e_{r}} \mid \mu \in M ; e_{1}, \ldots, e_{N} \in \mathbb{N}\right\}$ for $U_{\varphi, \geq}^{M}$ or similar PBW bases of decreasing ordered monomials; the same construction also provide PBW bases for $U_{-}, U_{0}^{M}$, and $U_{+}$.

Now, for every monomial $\mathcal{E}$ in the $E_{i}$ 's, let $s(\mathcal{E}):=\frac{1}{2} \varphi(w t(\mathcal{E})), r(\mathcal{E}):=$ $\frac{1}{2} r(\varphi(w t(\mathcal{E}))), \bar{r}(\mathcal{E}):=\frac{1}{2} \bar{r}(\varphi(w t(\mathcal{E})))$, where $w t(\mathcal{E})$ denotes the weight of $\mathcal{E}$ ( $E_{i}$ having weight $\left.\alpha_{i}\right)$, and similarly for every monomial $\mathcal{F}$ in the $F_{i}$ 's, $\left(F_{i}\right.$ having weight $\left.-\alpha_{i}\right)$. Then

$$
\pi_{\varphi}\left(\prod_{r=N}^{1} F_{\alpha^{r}}^{f_{r}} \cdot L_{\mu}, \prod_{r=N}^{1} E_{\alpha^{r}}^{e_{r}} \cdot L_{\nu}\right)=
$$




$$
\begin{aligned}
& =q^{-\left(r(\mu)-r\left(\prod_{r=N}^{1} F_{\alpha^{r}}^{f_{r}}\right) \mid \nu-s\left(\prod_{r=N}^{1} E_{\alpha^{r}}^{e_{r}}\right)\right)} \prod_{r=1}^{N} \delta_{e_{r}, f_{r}} \frac{\left[e_{r}\right]_{q_{\alpha^{r}}} ! q_{\alpha^{r}}^{+\left(\begin{array}{c}
e_{r} \\
2
\end{array}\right)}}{\left(q_{\alpha^{r}}^{-1}-q_{\alpha^{r}}\right)^{e_{r}}} \\
& \overline{\pi_{\varphi}}\left(L_{\mu} \cdot \prod_{r=N}^{1} E_{\alpha^{r}}^{e_{r}}, L_{\nu} \cdot \prod_{r=N}^{1} F_{\alpha^{r}}^{f_{r}}\right)= \\
& =q^{\left(\bar{r}(\mu)-\bar{r}\left(\prod_{r=N}^{1} E_{\alpha^{r}}^{f_{r}}\right) \mid \nu-s\left(\prod_{r=N}^{1} F_{\alpha^{r}}^{e_{r}}\right)\right)} \prod_{r=1}^{N} \delta_{e_{r}, f_{r}} \frac{\left[e_{r}\right]_{q_{\alpha^{r}}} ! q_{\alpha^{r}}^{-\left(\begin{array}{c}
e_{r} \\
2
\end{array}\right)}}{\left(q_{\alpha^{r}}-q_{\alpha^{r}}^{-1}\right)^{e_{r}}}
\end{aligned}
$$

gives the values of DRT pairings on PBW monomials (cf. [CV-1], Lemma 3.5 , and $[\mathbf{C V - 2}], \S 1$, up to normalizations). Now define modified root vectors $F_{\alpha}^{\varphi}:=L_{\tau_{\alpha}} F_{\alpha}=F_{\alpha} L_{\tau_{\alpha}}, E_{\alpha}^{\varphi}:=L_{\tau_{\alpha}} E_{\alpha}=E_{\alpha} L_{\tau_{\alpha}}$ for all $\alpha \in R^{+}$(and set $\left.F_{i}^{\varphi}:=F_{\alpha_{i}}^{\varphi}, F_{i}^{\varphi}:=F_{\alpha_{i}}^{\varphi}\right)$. Then

$$
\begin{aligned}
& \pi_{\varphi}\left(\prod_{r=N}^{1}\left(F_{\alpha^{r}}^{\varphi^{r}} f^{f_{r}} \cdot L_{(1+\varphi)(\mu)}, \prod_{r=N}^{1} E_{\alpha^{r}}^{e_{r}} \cdot L_{\nu}\right)=\right. \\
& =q^{-\left(\mu \mid \nu-s\left(\prod_{r=N}^{1} E_{\alpha^{r}}^{e_{r}}\right)\right)-\sum_{h<k}\left(f_{h} \tau_{\alpha} \mid f_{k} \alpha^{k}\right)} \cdot \prod_{r=1}^{N} \delta_{e_{r}, f_{r}} \frac{\left[e_{r}\right]_{q_{\alpha^{r}}} ! q_{\alpha^{r}}^{+\left(\begin{array}{c}
e_{r} \\
2
\end{array}\right)}}{\left(q_{\alpha^{r}}^{-1}-q_{\alpha^{r}}\right)^{e_{r}}} \\
& \overline{\pi_{\varphi}}\left(L_{(1-\varphi)(\mu)} \cdot \prod_{r=N}^{1}\left(E_{\alpha^{r}}^{\varphi}\right)^{e_{r}}, L_{\nu} \cdot \prod_{r=N}^{1} F_{\alpha^{r}}^{f_{r}}\right)= \\
& =q^{+\left(\mu \mid \nu-s\left(\prod_{r=N}^{1} F_{\alpha^{r}}^{e_{r}}\right)\right)+\sum_{h<k}\left(e_{h} \tau_{\alpha^{h}} \mid e_{k} \alpha^{k}\right)} \prod_{r=1}^{N} \delta_{e_{r}, f_{r}} \frac{\left.\left[e_{r}\right]_{q_{\alpha^{r}}} ! q_{\alpha^{r}}^{-\left(e_{r}\right.}{ }^{e^{2}}\right)}{\left(q_{\alpha^{r}}-q_{\alpha^{r}}^{-1}\right)^{e_{r}}}
\end{aligned}
$$

(cf. [CV-1], Lemma 3.5, and [CV-2], Proposition 1.9). In the sequel $U_{\varphi,-}$, resp. $U_{\varphi,+}$, will be the $k(q)$-subalgebra of $U_{\varphi, \leq}^{M}$, resp. $U_{\varphi, \geq}^{M}$, generated by the $F_{i}^{\varphi}$ 's, resp. by the $E_{i}^{\varphi}$ 's $(i=1, \ldots, n)$; these too have PBW bases of ordered monomials of modified root vectors.

2.5. Integer forms. Let $X^{(m)}:=X^{m} /[m]_{q_{i}} !$ and $\left(\begin{array}{c}Y ; c \\ t\end{array}\right):=\prod_{s=1}^{t} \frac{q_{i}^{c-s+1} Y-1}{q_{i}^{s}-1}$ be the so-called "divided powers"; let $\mathfrak{U}_{\varphi, \leq}^{M}$ be the $k\left[q, q^{-1}\right]$-subalgebra of $U_{\varphi, \leq}^{M}$ generated by $\left\{F_{i}^{(m)},\left(\begin{array}{c}M_{i} ; c \\ t\end{array}\right), M_{i}^{-1} \mid m, c, t \in \mathbb{N} ; i=1, \ldots, n\right\}$. Then $\mathfrak{U}_{\varphi, \leq}^{M}$ is a Hopf subalgebra of $U_{\varphi, \leq}^{M},(\mathrm{cf}$. [CV-2]) having a PBW basis (as a $k\left[q, q^{-1}\right]$-module) of increasing ordered monomials

$$
\left\{\prod_{i=1}^{n}\left(\begin{array}{c}
M_{i} ; 0 \\
t_{i}
\end{array}\right) M_{i}^{-E n t\left(t_{i} / 2\right)} \cdot \prod_{r=1}^{N} F_{\alpha^{r}}^{\left(n_{r}\right)} \mid t_{1}, \ldots, t_{n}, n_{1}, \ldots, n_{N} \in \mathbb{N}\right\}
$$


and a similar PBW basis of decreasing ordered monomials; in particular $\mathfrak{U}_{\varphi, \leq}^{M}$ is a $k\left[q, q^{-1}\right]$-form of $U_{\varphi, \leq}^{M}$. Similarly we define the Hopf subalgebra $\mathfrak{U}_{\varphi, \geq}^{M}$ and locate PBW bases for it.

Let $\bar{E}_{\alpha^{r}}:=\left(q_{\alpha^{r}}-q_{\alpha^{r}}^{-1}\right) E_{\alpha^{r}}, \forall r=1, \ldots, N$, and let $\mathcal{U}_{\varphi,>}^{M}$ be the $k\left[q, q^{-1}\right]$-subalgebra of $U_{\varphi, \geq}^{P}$ generated by $\left\{\bar{E}_{\alpha^{1}}, \ldots, \bar{E}_{\alpha^{N}}\right\} \cup\left\{M_{1}^{ \pm}, \ldots, M_{n}^{ \pm}\right\}$; then (cf. [DKP], [DP]) $\mathcal{U}_{\varphi, \geq}^{\bar{M}}$ is a Hopf subalgebra of $U_{\varphi, \geq}^{M}$, having a PBW basis (as a $k\left[q, q^{-1}\right]$-module)

$$
\left\{\prod_{i=1}^{n} M_{i}^{t_{i}} \cdot \prod_{r=1}^{N} \bar{E}_{\alpha^{r}}^{n_{r}} \mid t_{1}, \ldots, t_{n} \in \mathbb{Z} ; n_{1}, \ldots, n_{N} \in \mathbb{N}\right\}
$$

of increasing ordered monomials and a similar PBW basis of decreasing ordered monomials; in particular $\mathcal{U}_{\varphi, \geq}^{M}$ is a $k\left[q, q^{-1}\right]$-form of $U_{\varphi, \geq}^{M}$. The same procedure yields the definition of the Hopf subalgebra $\mathcal{U}_{\varphi, \leq}^{M}$ and provides PBW bases for it. The same integer forms can also be constructed using modified root vectors instead of the usual ones, hence these integer forms have also modified PBW bases of ordered monomials in the $M_{i}$ 's and the modified root vectors. Similar constructions and results hold for the algebras $U_{\varphi,-}, U_{\varphi, 0}^{M}, U_{\varphi,+}$, providing integer forms $\mathfrak{U}_{\varphi,-}, \mathcal{U}_{\varphi,+}$, and so on. Finally, we have decompositions

$$
\begin{aligned}
& \mathfrak{U}_{\varphi, \leq}^{M} \cong \mathfrak{U}_{\varphi,-} \otimes \mathfrak{U}_{\varphi, 0}^{M} \cong \mathfrak{U}_{\varphi, 0}^{M} \otimes \mathfrak{U}_{\varphi,-}, \quad \mathfrak{U}_{\varphi, \geq}^{M} \cong \mathfrak{U}_{\varphi,+} \otimes \mathfrak{U}_{\varphi, 0}^{M} \cong \mathfrak{U}_{\varphi, 0}^{M} \otimes \mathfrak{U}_{\varphi,+} \\
& \mathcal{U}_{\varphi, \leq}^{M} \cong \mathcal{U}_{\varphi,-} \otimes \mathcal{U}_{\varphi, 0}^{M} \cong \mathcal{U}_{\varphi, 0}^{M} \otimes \mathcal{U}_{\varphi,-}, \quad \mathcal{U}_{\varphi, \geq}^{M} \cong \mathcal{U}_{\varphi,+} \otimes \mathcal{U}_{\varphi, 0}^{M} \cong \mathcal{U}_{\varphi, 0}^{M} \otimes \mathcal{U}_{\varphi,+} .
\end{aligned}
$$

2.6. $k\left[q, q^{-1}\right]$-duality among integer forms. The very definitions and (2.3) imply that integer forms of opposite "fonts" (namely $\mathfrak{U}$ or $\mathcal{U}$ ) are $k\left[q, q^{-1}\right]$-dual of each other in the following sense: for every DRT pairing, if we take $\mathfrak{U}$ on one side, then the form $\mathcal{U}$ on the other side coincides with the subset of all elements which paired with $\mathfrak{U}$ give a value in $k\left[q, q^{-1}\right]$; and similarly reverting the roles of $\mathfrak{U}$ and $\mathcal{U}$. For instance

$$
\begin{aligned}
\mathfrak{U}_{\varphi, 0}^{M} & =\left\{y \in U_{\varphi, 0}^{M} \mid \pi_{\varphi}\left(\mathcal{U}_{\varphi, 0}^{M^{\prime}}, y\right) \subseteq k\left[q, q^{-1}\right]\right\} \\
& =\left\{x \in U_{\varphi, 0}^{M} \mid \pi_{\varphi}\left(x, \mathcal{U}_{\varphi, 0}^{M^{\prime}}\right) \subseteq k\left[q, q^{-1}\right]\right\} \\
\mathcal{U}_{\varphi, 0}^{M} & =\left\{y \in U_{\varphi, 0}^{M} \mid \pi_{\varphi}\left(\mathfrak{U}_{\varphi, 0}^{M^{\prime}}, y\right) \subseteq k\left[q, q^{-1}\right]\right\} \\
& =\left\{x \in U_{\varphi, 0}^{M} \mid \overline{\pi_{\varphi}}\left(x, \mathfrak{U}_{\varphi, 0}^{M^{\prime}}\right) \subseteq k\left[q, q^{-1}\right]\right\} \\
\mathfrak{U}_{\varphi,-} & =\left\{x \in U_{\varphi,-} \mid \pi_{\varphi}\left(x, \mathcal{U}_{\varphi,+}\right) \subseteq k\left[q, q^{-1}\right]\right\} \\
& =\left\{y \in U_{\varphi,-} \mid \overline{\pi_{\varphi}}\left(\mathcal{U}_{\varphi,+}, y\right) \subseteq k\left[q, q^{-1}\right]\right\} \\
\mathcal{U}_{\varphi, \geq}^{M} & =\left\{x \in U_{\varphi, \geq}^{M} \mid \overline{\pi_{\varphi}}\left(x, \mathfrak{U}_{\varphi, \leq}^{M^{\prime}}\right) \subseteq k\left[q, q^{-1}\right]\right\}
\end{aligned}
$$




$$
=\left\{y \in U_{\varphi, \geq}^{M} \mid \pi_{\varphi}\left(\mathfrak{U}_{\varphi, \leq}^{M^{\prime}}, y\right) \subseteq k\left[q, q^{-1}\right]\right\}
$$

\section{The quantum group $U_{q, \varphi}^{M}(\mathfrak{g})$.}

3.1. The quantum double. Let $H_{-}, H_{+}$be two arbitrary Hopf algebras on a ground field (or ring) $F$, and let $\pi:\left(H_{-}\right)_{o p} \otimes H_{+} \rightarrow F$ be any arbitrary Hopf pairing. Then the Drinfel'd's quantum double $D=D\left(H_{-}, H_{+}, \pi\right)$ is the algebra $T\left(H_{-} \oplus H_{+}\right) / \mathcal{R}$, where $\mathcal{R}$ is the ideal of relations

$$
\begin{gathered}
1_{H_{-}}=1=1_{H_{+}}, \quad x \otimes y=x y \quad \text { for } x, y \in H_{+} \text {or } x, y \in H_{-} \\
\sum_{(x),(y)} \pi\left(y_{(2)}, x_{(2)}\right) x_{(1)} \otimes y_{(1)}=\sum_{(x),(y)} \pi\left(y_{(1)}, x_{(1)}\right) y_{(2)} \otimes x_{(2)} \\
\text { for } x \in H_{+}, y \in H_{-} .
\end{gathered}
$$

Then (cf. [DL], Theorem 3.6) $D$ has a canonical structure of Hopf algebra such that $H_{-}, H_{+}$are Hopf subalgebras of it and multiplication yields isomorphisms of coalgebras

$$
H_{+} \otimes H_{-} \longleftrightarrow D \otimes D \stackrel{m}{\longrightarrow} D, \quad H_{-} \otimes H_{+} \longleftrightarrow D \otimes D \stackrel{m}{\longrightarrow} D .
$$

Now consider $D_{q, \varphi}^{M}(\mathfrak{g}):=D\left(U_{\varphi, \leq}^{Q}, U_{\varphi, \geq}^{M}, \pi_{\varphi}\right)$; by definition, $D_{q, \varphi}^{M}(\mathfrak{g})$ is generated by $K_{\alpha}, L_{\mu}, F_{i}, E_{i}$ - identified with $1 \otimes K_{\alpha}, L_{\mu} \otimes 1,1 \otimes F_{i}, E_{i} \otimes 1$ via $D_{q, \varphi}^{M}(\mathfrak{g}) \cong U_{\varphi, \geq}^{M} \otimes U_{\varphi, \leq}^{Q}-(\alpha \in Q, \mu \in M, i=1, \ldots, n)$, while the relations defining $\mathcal{R}$ reduce to

$$
\begin{gathered}
K_{\alpha} L_{\mu}=L_{\mu} K_{\alpha}, \quad K_{\alpha} E_{j}=q_{i}^{+\left(\alpha_{j} \mid \alpha\right)} E_{j} K_{\alpha}, \quad L_{\mu} F_{j}=q_{i}^{-\left(\alpha_{j} \mid \mu\right)} F_{j} L_{\mu} \\
E_{i} F_{j}-F_{j} E_{i}=\delta_{i j} \frac{L_{\alpha_{i}}-K_{-\alpha_{i}}}{q_{i}-q_{i}^{-1}} .
\end{gathered}
$$

Finally, PBW bases of quantum Borel algebras provide PBW bases of $D_{q, \varphi}^{M}(\mathfrak{g})$. In the sequel we shall also use the notation $D_{M}:=D_{q, \varphi}^{M}(\mathfrak{g})$.

3.2. The quantum algebra $U_{q, \varphi}^{M}(\mathfrak{g})$. Let $\mathfrak{K}_{\varphi}^{M}$ be the ideal of $D_{q, \varphi}^{M}(\mathfrak{g})$ generated by the elements $L \otimes 1-1 \otimes L, L \in U_{\varphi, 0}^{M} ; \mathfrak{K}_{\varphi}^{M}$ is in fact a Hopf ideal, whence $D_{q, \varphi}^{M}(\mathfrak{g}) / \mathfrak{K}_{\varphi}^{M}$ is a Hopf algebra. Then from above we get a presentation of $U_{q, \varphi}^{M}(\mathfrak{g}):=D_{q, \varphi}^{M}(\mathfrak{g}) / \mathfrak{K}_{\varphi}^{M}$ : It is the associative $k(q)$-algebra with 1 given by generators $F_{i}, L_{\mu}, E_{i}$ and relations

$$
\begin{gathered}
L_{0}=1, \quad L_{\mu} L_{\nu}=L_{\mu+\nu}=L_{\nu} L_{\mu}, \\
L_{\mu} F_{i}=q^{-\left(\alpha_{j} \mid \mu\right)} F_{i} L_{\mu}, \quad L_{\mu} E_{i}=q^{+\left(\alpha_{j} \mid \mu\right)} E_{i} L_{\mu} \\
E_{i} F_{h}-F_{h} E_{i}=\delta_{i h} \frac{L_{\alpha_{i}}-L_{-\alpha_{i}}}{q_{i}-q_{i}^{-1}}
\end{gathered}
$$




$$
\begin{aligned}
& \sum_{k=0}^{1-a_{i j}}(-1)^{k}\left[\begin{array}{c}
1-a_{i j} \\
k
\end{array}\right]_{q_{i}} E_{i}^{1-a_{i j}-k} E_{j} E_{i}^{k}=0, \\
& \sum_{k=0}^{1-a_{i j}}(-1)^{k}\left[\begin{array}{c}
1-a_{i j} \\
k
\end{array}\right]_{q_{i}} F_{i}^{1-a_{i j}-k} F_{j} F_{i}^{k}=0
\end{aligned}
$$

(for all $\mu \in M, i, j, h=1, \ldots, n$ with $i \neq j$ ) with the Hopf structure given by

$$
\begin{array}{ccc}
\Delta_{\varphi}\left(F_{i}\right)=F_{i} \otimes L_{-\alpha_{i}-\tau_{i}}+L_{\tau_{i}} \otimes F_{i}, & \epsilon_{\varphi}\left(F_{i}\right)=0, & S_{\varphi}\left(F_{i}\right)=-F_{i} L_{\alpha_{i}} \\
\Delta_{\varphi}\left(L_{\mu}\right)=L_{\mu} \otimes L_{\mu}, & \epsilon_{\varphi}\left(L_{\mu}\right)=1, & S_{\varphi}\left(L_{\mu}\right)=L_{-\mu} \\
\Delta_{\varphi}\left(E_{i}\right)=E_{i} \otimes L_{\tau_{i}}+L_{\alpha_{i}-\tau_{i}} \otimes F_{i}, & \epsilon_{\varphi}\left(E_{i}\right)=0, & S_{\varphi}\left(F_{i}\right)=-L_{-\alpha_{i}} E_{i} .
\end{array}
$$

For $\varphi=0$ one recovers the usual one-parameter quantum enveloping algebras. Finally we let $p r_{M}: D_{q, \varphi}^{M}(\mathfrak{g}) \longrightarrow D_{q, \varphi}^{M}(\mathfrak{g}) / \mathfrak{K}_{\varphi}^{M}=: U_{q, \varphi}^{M}(\mathfrak{g})$ be the canonical Hopf algebra epimorphism; we shall also use notation $K_{\alpha}:=L_{\alpha}$, $M_{\mu}:=L_{\mu}, \forall \alpha \in Q, \mu \in M$.

3.3. Integer forms of $U_{q, \varphi}^{M}(\mathfrak{g})$. Let $\mathfrak{U}_{\varphi}^{M}(\mathfrak{g})$ be the $k\left[q, q^{-1}\right]$-subalgebra of $U_{q, \varphi}^{M}(\mathfrak{g})$ generated by $\left\{F_{i}^{(\ell)},\left(\begin{array}{c}M_{i} ; c \\ t\end{array}\right), M_{i}^{-1}, E_{i}^{(m)} \mid \ell, c, t, m \in \mathbb{N} ; i=1, \ldots, n\right\}$; this is a Hopf subalgebra of $U_{q, \varphi}^{M}(\mathfrak{g})$ (cf. [DL], $\S 3$ ), with PBW basis (over $\left.k\left[q, q^{-1}\right]\right)$

$$
\left\{\prod_{r=N}^{1} E_{\alpha^{r}}^{\left(n_{r}\right)} \cdot \prod_{i=1}^{n}\left(\begin{array}{c}
M_{i} ; 0 \\
t_{i}
\end{array}\right) M_{i}^{-E n t\left(t_{i} / 2\right)} \cdot \prod_{r=1}^{N} F_{\alpha^{r}}^{\left(m_{r}\right)} \mid n_{r}, t_{i}, m_{r} \in \mathbb{N}, \forall r, i\right\} ;
$$

this is also a $k(q)$-basis of $U_{q, \varphi}^{M}(\mathfrak{g})$, hence $\mathfrak{U}^{M}(\mathfrak{g})$ is a $k\left[q, q^{-1}\right]$-form of $U_{q, \varphi}^{M}(\mathfrak{g})$. $\S 12)$

Let $\mathcal{U}_{\varphi}^{M}(\mathfrak{g})$ be the $k\left[q, q^{-1}\right]$-subalgebra of $U_{q, \varphi}^{M}(\mathfrak{g})$ generated by (cf. [DP],

$$
\left\{\bar{F}_{\alpha^{1}}, \ldots, \bar{F}_{\alpha^{N}}\right\} \cup\left\{M_{1}^{ \pm 1}, \ldots, M_{n}^{ \pm 1}\right\} \cup\left\{\bar{E}_{\alpha^{1}}, \ldots, \bar{E}_{\alpha^{N}}\right\} ;
$$

this is a Hopf subalgebra of $U_{q, \varphi}^{P}(\mathfrak{g})$, having a PBW basis (over $k\left[q, q^{-1}\right]$ )

$$
\left\{\prod_{r=N}^{1} \bar{E}_{\alpha^{r}}^{n_{r}} \cdot \prod_{i=1}^{n} M_{i}^{t_{i}} \cdot \prod_{r=1}^{N} \bar{F}_{\alpha^{r}}^{m_{r}} \mid t_{i} \in \mathbb{Z}, n_{r}, m_{r} \in \mathbb{N}, \forall i, r\right\} ;
$$

the latter is also a $k(q)$-basis of $U_{q, \varphi}^{M}(\mathfrak{g})$, hence $\mathcal{U}_{\varphi}^{M}(\mathfrak{g})$ is a $k\left[q, q^{-1}\right]$-form of $U_{q, \varphi}^{M}(\mathfrak{g})$.

Like for quantum Borel algebras, the same forms can also be defined using modified root vectors, hence they have also PBW bases of ordered monomials in the $M_{i}$ 's and the modified root vectors. 
3.4. Specialization at roots of 1 and quantum Frobenius morphisms. When dealing with specializations, if any scalar $c \in k \backslash\{0\}$ is fixed then $k$ is thought of as a $k\left[q, q^{-1}\right]$-algebra via $k \cong k\left[q, q^{-1}\right] /(q-c)$.

Let $\varepsilon$ be a primitive $\ell$-th root of 1 , for $\ell$ odd, $\ell>d:=\max _{i}\left\{d_{i}\right\}_{i}$, or $\ell=1$. Then we set $\mathfrak{U}_{\varepsilon, \varphi}^{M}(\mathfrak{g}):=\mathfrak{U}_{\varphi}^{M}(\mathfrak{g}) /(q-\varepsilon) \mathfrak{U}_{\varphi}^{M}(\mathfrak{g}) \cong \mathfrak{U}_{\varphi}^{M}(\mathfrak{g}) \otimes_{k\left[q, q^{-1}\right]} k$. When $\ell=1$ (i.e., $\varepsilon=1$ ) it is well-known (cf. e.g., $[\mathbf{C V - 2}]$ or $[\mathbf{D L}])^{2}$ that $\mathfrak{U}_{1, \varphi}^{M}(\mathfrak{g})$ is a Poisson Hopf coalgebra, and we have a Poisson Hopf coalgebra isomorphism

$$
\mathfrak{U}_{1, \varphi}^{M}(\mathfrak{g}) \cong U\left(\mathfrak{g}^{\tau}\right)
$$

in a word, $\mathfrak{U}_{\varphi}^{M}(\mathfrak{g})$ specializes to $U\left(\mathfrak{g}^{\tau}\right)$ for $q \rightarrow 1:$ In symbols, $\mathfrak{U}_{\varphi}^{M}(\mathfrak{g}) \stackrel{q \rightarrow 1}{\longrightarrow}$ $U\left(\mathfrak{g}^{\tau}\right)$.

When $\ell>1$, from $[\mathbf{C V - 2}], \S 3.2$ (cf. also $[\mathbf{L u}]$, [DL]) we have an epimorphism

$$
\mathfrak{F r}_{\mathfrak{g}^{\tau}}: \mathfrak{U}_{\varepsilon, \varphi}^{M}(\mathfrak{g}) \longrightarrow \mathfrak{U}_{1, \varphi}^{M}(\mathfrak{g}) \cong U\left(\mathfrak{g}^{\tau}\right)
$$

of Hopf algebras defined by (recall that $M_{i}:=L_{\mu_{i}}$ )

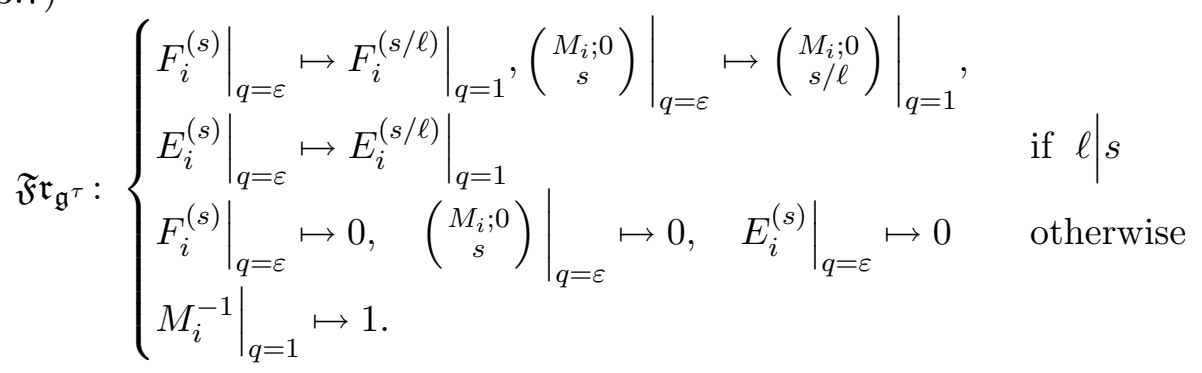

If $\varphi=0$ - whence $\tau=0-$ and $\ell=p$ is prime, it is shown in $[\mathbf{L u}]$, $\S 8.15$, that $\mathfrak{F r}_{\mathfrak{g}^{0}}$ (for $M=Q$ ) can be regarded as a lifting of the Frobenius morphism $G_{\mathbb{Z}_{p}} \rightarrow G_{\mathbb{Z}_{p}}$ to characteristic zero; for this reason, we refer to $\mathfrak{F r}_{\mathfrak{g}^{\tau}}$ as a quantum Frobenius morphism.

Similarly, we set $\mathcal{U}_{\varepsilon, \varphi}^{M}(\mathfrak{g}):=\mathcal{U}_{\varphi}^{M}(\mathfrak{g}) /(q-\varepsilon) \mathcal{U}_{\varphi}^{M}(\mathfrak{g}) \cong \mathcal{U}_{\varphi}^{M}(\mathfrak{g}) \otimes_{k\left[q, q^{-1}\right]} k ;$ when $\ell=1$ it is known (cf. [DP], Theorem 12.1, and [DKP], Remark $7.7(\mathrm{c}))$ that

$$
\mathcal{U}_{1, \varphi}^{M}(\mathfrak{g}) \cong F\left[H_{M}^{\tau}\right]
$$

as Poisson Hopf algebras over $k$ : Here $H_{M}^{\tau}$ is the connected Poisson group with tangent Lie bialgebra $\mathfrak{h}^{\tau}$ - defined in $\S 1.2$ - and $M$ the character group of a maximal torus. In a word, $\mathcal{U}_{\varphi}^{M}(\mathfrak{g})$ specializes to $F\left[H_{M}^{\tau}\right]$ as $q \rightarrow 1$,

\footnotetext{
${ }^{2}$ This result is more general than in $[\mathbf{C V}-\mathbf{2}]$ or $[\mathbf{D L}]$ : It can be proved on the same lines of Theorem 7.2 below.
} 
or $\mathcal{U}_{\varphi}^{M}(\mathfrak{g}) \stackrel{q \rightarrow 1}{\longrightarrow} F\left[H_{M}^{\tau}\right]$. When $\ell>1$, from $[\mathbf{D K P}], \S \S 7.6-7$ we record the existence of a Hopf algebra monomorphism

$$
\mathcal{F} r_{\mathfrak{g}^{\tau}}: F\left[H_{M}^{\tau}\right] \cong \mathcal{U}_{1, \varphi}^{M}(\mathfrak{g}) \smile \mathcal{U}_{\varepsilon, \varphi}^{M}(\mathfrak{g})
$$

(cf. also [DP] for the one-parameter case) defined by $\left(\alpha \in R^{+}, \mu \in M\right)$

$$
\mathcal{F} r_{\mathfrak{g}^{\tau}}:\left.\left.\quad \bar{F}_{\alpha}\right|_{q=1} \mapsto \bar{F}_{\alpha}^{\ell}\right|_{q=\varepsilon},\left.\left.\quad L_{\mu}\right|_{q=1} \mapsto L_{\mu}^{\ell}\right|_{q=\varepsilon},\left.\left.\quad \bar{E}_{\alpha}\right|_{q=1} \mapsto \bar{E}_{\alpha}^{\ell}\right|_{q=\varepsilon} .
$$

Again, we refer to $\mathcal{F} r_{\mathfrak{g}^{\tau}}$ as a quantum Frobenius morphism: If $\varphi=0$ and $\ell=p$ is prime it is a lifting of the Frobenius morphism $H_{\mathbb{Z}_{p}} \rightarrow H_{\mathbb{Z}_{p}}$ to characteristic zero ${ }^{3}$.

\section{Quantum function algebras}

4.1. The quantum function algebras $F_{q, \varphi}^{M}\left[B_{ \pm}\right]$. Let $F_{q, \varphi}^{M}\left[B_{ \pm}\right]$be the quantum function algebra relative to $U_{q, \varphi}^{M^{\prime}}\left(\mathfrak{b}_{ \pm}\right)$, defined as the algebra of matrix coefficients of positive ${ }^{4}$ finite dimensional representations of $U_{q, \varphi}^{M^{\prime}}\left(\mathfrak{b}_{ \pm}\right)$. Then $F_{q, \varphi}^{M}\left[B_{ \pm}\right]$is a Hopf algebra, which we call dual of $U_{q, \varphi}^{M^{\prime}}\left(\mathfrak{b}_{ \pm}\right)$for there is a perfect Hopf pairing (evaluation) among them; in fact $F_{q, \varphi}^{M}\left[B_{ \pm}\right]$is a Hopf subalgebra of $U_{q, \varphi}^{M^{\prime}}\left(\mathfrak{b}_{ \pm}\right)^{\circ}$ (the - restricted - Hopf dual of $U_{q, \varphi}^{M^{\prime}}\left(\mathfrak{b}_{ \pm}\right)$, in the sense of $[\mathbf{S w}]$, Ch. VI). The DRT pairings provide Hopf algebra isomorphisms

$$
F_{q, \varphi}^{M}\left[B_{+}\right] \cong\left(U_{\varphi, \leq}^{M^{\prime}}\right)_{o p}, \quad F_{q, \varphi}^{M}\left[B_{-}\right] \cong\left(U_{\varphi, \geq}^{M^{\prime}}\right)_{o p}
$$

induced by the pairing $\pi_{\varphi}$, resp. $\overline{\pi_{\varphi}}$; by means of these, the DRT pairings can be seen as natural evaluation pairings (cf. [DL], $\S 4$, and [CV-2], $\S \S 2-3$ ).

4.2. Integer forms of $F_{q, \varphi}^{M}\left[B_{ \pm}\right]$. Let

$$
\begin{aligned}
& \mathfrak{F}_{\varphi}^{M}\left[B_{ \pm}\right]:=\left\{f \in F_{q, \varphi}^{M}\left[B_{ \pm}\right] \mid\left\langle f, \mathfrak{U}_{\varphi}^{M^{\prime}}\left(\mathfrak{b}_{ \pm}\right)\right\rangle \subseteq k\left[q, q^{-1}\right]\right\} \\
& \mathcal{F}_{\varphi}^{M}\left[B_{ \pm}\right]:=\left\{f \in F_{q, \varphi}^{M}\left[B_{ \pm}\right] \mid\left\langle f, \mathcal{U}_{\varphi}^{M^{\prime}}\left(\mathfrak{b}_{ \pm}\right)\right\rangle \subseteq k\left[q, q^{-1}\right]\right\}
\end{aligned}
$$

where $\langle\rangle:, F_{q, \varphi}^{M}\left[B_{ \pm}\right] \otimes U_{q, \varphi}^{M^{\prime}}\left(\mathfrak{b}_{ \pm}\right) \rightarrow k(q)$ is the natural evaluation pairing; then

$$
\mathfrak{F}_{\varphi}^{M}\left[B_{ \pm}\right] \cong\left(\mathcal{U}_{\varphi}^{M}\left(\mathfrak{b}_{\mp}\right)\right)_{o p}, \quad \mathcal{F}_{\varphi}^{M}\left[B_{ \pm}\right] \cong\left(\mathfrak{U}_{\varphi}^{M}\left(\mathfrak{b}_{\mp}\right)\right)_{o p},
$$

\footnotetext{
${ }^{3}$ Here $H_{\mathbb{Z}_{p}}$ denotes the Chevalley-type group-scheme over $\mathbb{Z}_{p}$ associated to $H$.

${ }^{4}$ Namely those having a basis on which the $L_{\nu}$ 's $\left(\nu \in M^{\prime}\right)$ act diagonally by powers of
} $q$. 
because of $\S 2.6$ and (4.1): In particular $\mathfrak{F}_{\varphi}^{M}\left[B_{ \pm}\right]$and $\mathcal{F}_{\varphi}^{M}\left[B_{ \pm}\right]$are integer forms of $F_{q, \varphi}^{M}\left[B_{ \pm}\right]$.

\subsection{The quantum function algebra $F_{q, \varphi}^{M}[G]$ and its integer forms.} Like in $\S 4.1$, we define the quantum function algebra $F_{q, \varphi}^{M}[G]$ (relative to $\left.U_{q, \varphi}^{M^{\prime}}(\mathfrak{g})\right)$ to be the algebra of matrix coefficients of positive finite dimensional representations of $U_{q, \varphi}^{M^{\prime}}(\mathfrak{g})$ (cf. [DL], $\S 4$, and $[\mathbf{C V - 2}], \S 2.1$ ); it is a Hopf subalgebra of $U_{q, \varphi}^{M^{\prime}}(\mathfrak{g})^{\circ}$, perfectly paired with $U_{q, \varphi}^{M^{\prime}}(\mathfrak{g})$ by the natural evaluation pairing (whence we call it dual of $U_{q, \varphi}^{M^{\prime}}(\mathfrak{g})$ ). As for integer forms, let

$$
\begin{aligned}
& \mathfrak{F}_{\varphi}^{M}[G]:=\left\{f \in F_{q, \varphi}^{M}[G] \mid\left\langle f, \mathfrak{U}_{\varphi}^{M^{\prime}}(\mathfrak{g})\right\rangle \subseteq k\left[q, q^{-1}\right]\right\} \\
& \mathcal{F}_{\varphi}^{M}[G]:=\left\{f \in F_{q, \varphi}^{M}[G] \mid\left\langle f, \mathcal{U}_{\varphi}^{M^{\prime}}(\mathfrak{g})\right\rangle \subseteq k\left[q, q^{-1}\right]\right\}
\end{aligned}
$$

where $\langle\rangle:, F_{q, \varphi}^{M}[G] \otimes U_{q, \varphi}^{M^{\prime}}(\mathfrak{g}) \rightarrow k(q)$ is the natural evaluation pairing; we shall later prove that these are $k\left[q, q^{-1}\right]$-integer forms (as Hopf subalgebras) of $F_{q, \varphi}^{M}[G]$.

4.4. Specialization at roots of 1 . Let $\varepsilon$ be a primitive $\ell$-th root of 1 in $k$ (with the assumptions of $\S 3.4$ on $\ell$ ), and set $\mathfrak{F}_{\varepsilon, \varphi}^{M}[G]:=\mathfrak{F}_{\varphi}^{M}[G] /(q-$ $\varepsilon) \mathfrak{F}_{\varphi}^{M}[G] \cong \mathfrak{F}_{\varphi}^{M}[G] \otimes_{k\left[q, q^{-1}\right]} k$. For $\ell=1$, we have $\mathfrak{F}_{1, \varphi}^{M}[G] \cong F\left[G_{M}^{\tau}\right]$ as Poisson Hopf $k$-algebras (cf. [CV-2], [DL]), i.e.,

$$
\mathfrak{F}_{\varphi}^{M}[G] \stackrel{q \rightarrow 1}{\longrightarrow} F\left[G_{M}^{\tau}\right] ;
$$

here $G_{M}^{\tau}$ is the connected Poisson group with tangent Lie bialgebra $\mathfrak{g}^{\tau}$ and $M$ as character group of a maximal torus. In fact this result arises as dual of $\mathfrak{U}_{\varphi}^{M^{\prime}}(\mathfrak{g}) \stackrel{q \rightarrow 1}{\longrightarrow} U\left(\mathfrak{g}^{\tau}\right)$. When $\ell>1$, another quantum Frobenius morphism, namely a Hopf algebra monomorphism

$$
\mathfrak{F r}_{G^{\tau}}: F\left[G_{M}^{\tau}\right] \cong \mathfrak{F}_{1, \varphi}^{M}[G] \smile \mathfrak{F}_{\varepsilon, \varphi}^{M}[G],
$$

is defined (cf. [CV-2], §3.3), which is dual of $\mathfrak{F r}_{\mathfrak{g}^{\tau}}: \mathfrak{U}_{\varepsilon, \varphi}^{M^{\prime}}(\mathfrak{g}) \longrightarrow \mathfrak{U}_{1, \varphi}^{M^{\prime}}(\mathfrak{g}) \cong$ $U\left(\mathfrak{g}^{\tau}\right)$.

\section{Quantum formal groups.}

5.1. Formal Hopf algebras and quantum formal groups. In this subsection we introduce the notion of quantum formal group. Recall (cf. [Di], Ch. I) that formal groups can be defined in a category of a special type of commutative topological algebras, whose underlying vector space (or module) is linearly compact; following Drinfel'd's philosophy,we define quantum formal groups by simply dropping out any commutativity assumption of the classical notion of formal group; thus now we quickly outline how to modify the latter (following [Di], Ch. I) in order to define our new quantum objects. 
Let $E$ be any vector space over a field $K$ (one can then generalize more or less wathever follows to the case of free modules over a ring), and let $E^{*}$ be its (linear) dual; we write $\left\langle x^{*}, x\right\rangle$ for $x^{*}(x)$ for $x \in E, x^{*} \in E^{*}$. We consider on $E^{*}$ the weak *-topology, i.e., the coarsest topology such that for each $x \in E$ the linear map $x^{*} \mapsto\left\langle x^{*}, x\right\rangle$ of $E^{*}$ into $K$ is continuous, when $K$ is given the discrete topology. We can describe this topology by choosing a basis $\left\{e_{i}\right\}_{i \in I}$ of $E$ : To each $i \in I$ we associate the linear (coordinate) form $e_{i}^{*}$ on $E$ such that $\left\langle e_{i}^{*}, e_{j}\right\rangle=\delta_{i j}$, and we say that the family $\left\{e_{i}^{*}\right\}_{i \in I}$ is the pseudobasis of $E^{*}$ dual to $\left\{e_{i}\right\}_{i \in I}$; then the subspace $E^{\prime}$ of $E$ which is (algebraically) generated by the $e_{i}^{*}$ is dense in $E^{*}$, and $E^{*}$ is nothing but the completion of $E^{\prime}$, when $E^{\prime}$ is given the topology for which a fundamental system of neighborhoods of 0 consists of the vector subspaces containing almost all the $e_{i}^{*}$; thus elements of $E^{*}$ can be described by series in the $e_{i}^{*}$ 's which in the given topology are in fact convergent. Finally, the topological vector spaces $E^{*}$ are characterized by the property of linear compactness.

Let now $E, F$ be any two vector spaces over $K$, and $u: E \rightarrow F$ a linear map; then the dual map $u^{*}: F^{*} \rightarrow E^{*}$ is continuous, and conversely for any linear map $v: F^{*} \rightarrow E^{*}$ which is continuous there exists a unique linear map $u: E \rightarrow F$ such that $v=u^{*}$.

The tensor product $E^{*} \otimes F^{*}$ is naturally identified to a subspace of $(E \otimes F)^{*}$ by $\left\langle x^{*} \otimes y^{*}, x \otimes y\right\rangle=\left\langle x^{*}, x\right\rangle \cdot\left\langle y^{*}, y\right\rangle$; thus if $\left\{e_{i}\right\}_{i \in I}$ and $\left\{f_{j}\right\}_{j \in J}$ are bases of $E$ and $F$, and $\left\{e_{i}^{*}\right\}_{i \in I}$ and $\left\{f_{j}^{*}\right\}_{j \in J}$ are their dual pseudobases in $E^{*}$ and $F^{*}$, then $\left\{e_{i}^{*} \otimes f_{j}^{*}\right\}_{i \in I, j \in J}$ is the dual pseudobasis of $\left\{e_{i} \otimes f_{j}\right\}_{i \in I, j \in J}$ in $(E \otimes F)^{*}$. Thus $(E \otimes F)^{*}$ is the completion of $E^{*} \otimes F^{*}$ for the tensor product topology, i.e., the topology of $E^{*} \otimes F^{*}$ for which a fundamental system of neighborhoods of 0 consists of the sets $E^{*} \otimes V+W \otimes F^{*}$ where $V$, resp. $W$, ranges in a fundamental system of neighborhoods of 0 made of vector subspaces; we denote this completion by $E^{*} \widehat{\otimes} F^{*}$, and we call it the completed (or topological) tensor product of $E^{*}$ and $F^{*}$; the embedding $E^{*} \otimes F^{*} \longleftrightarrow(E \otimes F)^{*}=E^{*} \widehat{\otimes} F^{*}$ is then continuous. Finally, if $u: E_{1} \rightarrow E_{2}, v: F_{1} \rightarrow F_{2}$ are linear maps, then $(u \otimes v)^{*}:\left(E_{2} \otimes F_{2}\right)^{*}=$ $E_{2}{ }^{*} \widehat{\otimes} F_{2}{ }^{*} \longrightarrow\left(E_{1} \otimes F_{1}\right)^{*}=E_{1}{ }^{*} \widehat{\otimes} F_{1}{ }^{*}$ coincides with the continuous extension to $E_{2}{ }^{*} \widehat{\otimes} F_{2}{ }^{*}$ of the continuous map $u^{*} \otimes v^{*}: E_{2}{ }^{*} \otimes F_{2}{ }^{*} \rightarrow E_{1}{ }^{*} \otimes F_{1}{ }^{*}$; thus it is also denoted by $u^{*} \widehat{\otimes} v^{*}$.

We define a linearly compact algebra to be a topological algebra whose underlying vector space (or free module) is linearly compact: Then linearly compact algebras form a full subcategory of the category of topological algebras; morever, for any two objects $A_{1}$ and $A_{2}$ in this category, their topological tensor product $A_{1} \widehat{\otimes} A_{2}$ is defined. Dually, within the category of linearly compact vector spaces we define linearly compact coalgebras as triplets $(C, \Delta, \epsilon)$ with $\Delta: C \rightarrow C \widehat{\otimes} C$ and $\epsilon: C \rightarrow K$ satisfying the usual 
coalgebra axioms. The arguments in [Di] (which never require commutativity nor cocommutativity) show that ()$^{*}:(A, m, 1) \mapsto\left(A^{*}, m^{*}, 1^{*}\right)$ defines a contravariant functor from algebras to linearly compact coalgebras, while ()$^{*}:(C, \Delta, \epsilon) \mapsto\left(C^{*}, \Delta^{*}, \epsilon^{*}\right)$ defines a contravariant functor from coalgebras to linearly compact algebras. Finally, we define a formal Hopf algebra as a datum $(H, m, 1, \Delta, \epsilon, S)$ such that $(H, m, 1)$ is a linearly compact algebra, $(H, \Delta, \epsilon)$ is a linearly compact coalgebra, and the usual compatibility axioms of Hopf algebras are satisfied. "Usual" Hopf algebras are particular cases of formal Hopf algebras.

We define quantum formal group the spectrum of a formal Hopf algebra (whereas classical formal groups are spectra of commutative formal Hopf algebras: cf. [Di], Ch. I).

Our goal is to study $U_{q, \varphi}^{M}(\mathfrak{g})^{*}$. Since $U_{q, \varphi}^{M}(\mathfrak{g})$ is a Hopf algebra, its linear dual $U_{q, \varphi}^{M}(\mathfrak{g})^{*}$ is a formal Hopf algebra. The functor ( ) turns the natural epimorphism $p r_{M}: D_{M} \longrightarrow U_{q, \varphi}^{M}(\mathfrak{g})$ into a monomorphism $j_{M^{\prime}}:=$ $\left(p r_{M}\right)^{*}: U_{q, \varphi}^{M}(\mathfrak{g})^{*} \longleftrightarrow D_{M}{ }^{*}$ of formal Hopf algebras: Therefore we begin by studying $D_{M}{ }^{*}$. The following is straightforward:

Proposition 5.2. Let $H_{-}, H_{+}$be Hopf F-algebras, let $\pi:\left(H_{-}\right)_{o p} \otimes$ $H_{+} \longrightarrow F$ be an arbitrary Hopf pairing, and let $D:=D\left(H_{-}, H_{+}, \pi\right)$ be the corresponding quantum double. Then there exist F-algebra isomorphisms

$$
D^{*} \cong H_{+}{ }^{*} \widehat{\otimes} H_{-}{ }^{*}, \quad D^{*} \cong H_{-}{ }^{*} \widehat{\otimes} H_{+}{ }^{*}
$$

dual of the $F$-coalgebra isomorphisms $D \cong H_{+} \otimes H_{-}, D \cong H_{-} \otimes H_{+}$ (cf. $\S 3.1)$.

5.3. Quantum enveloping algebras as function algebras. The DRT pairings induce several linear embeddings, namely

$U_{\varphi,-} \longleftrightarrow U_{\varphi,+}{ }^{*}, \quad i m_{M}: U_{\varphi, 0}^{M} \longleftrightarrow U_{\varphi, 0}^{M^{*}}, \quad U_{\varphi, \leq}^{M} \longleftrightarrow U_{\varphi, \geq}^{M^{\prime} *} \quad$ (induced by $\pi_{\varphi}$ ) $U_{\varphi,+} \longleftrightarrow U_{\varphi,-}{ }^{*}, \quad \overline{i m}_{M}: U_{\varphi, 0}^{M} \longleftrightarrow U_{\varphi, 0}^{M^{\prime}}{ }^{*}, \quad U_{\varphi, \geq}^{M} \longleftrightarrow U_{\varphi, \leq}^{M^{\prime}}{ }^{*} \quad$ (induced by $\overline{\pi_{\varphi}}$ ) the right-hand-side ones being also embeddings of formal Hopf algebras. Therefore we identify the various quantum algebras with their images in the corresponding dual spaces.

\section{Lemma 5.4.}

(a) The subset $\left\{\prod_{r=N}^{1}(-1)^{f_{r}} q_{\alpha^{r}}^{-\left(\begin{array}{c}f_{r} \\ 2\end{array}\right)}\left(\bar{F}_{\alpha^{r}}^{\varphi}\right)^{f_{r}} \mid f_{1}, \ldots, f_{N} \in \mathbb{N}\right\}$ of $\mathcal{U}_{\varphi,-}$ is the pseudobasis of $U_{+}^{*}$ dual of the PBW basis of $\mathfrak{U}_{+}$of decreasing ordered monomials, while the subset $\left\{\prod_{r=N}^{1}(-1)^{f_{r}} q_{\alpha^{r}}^{-\left(\begin{array}{c}f_{r} \\ 2\end{array}\right)}\left(F_{\alpha^{r}}^{\varphi}\right)^{\left(f_{r}\right)} \mid f_{1}, \ldots, f_{N} \in \mathbb{N}\right\}$ of $\mathfrak{U}_{\varphi,-}$ is the pseudobasis of $U_{+}^{*}$ dual of the PBW basis of $\mathcal{U}_{+}$of decreasing ordered monomials. A similar statement holds with the roles of $U_{-}$and $U_{+}$ reversed. 
(b) $\mathcal{U}_{\varphi, 0}^{M}$ (hence $U_{\varphi, 0}^{M}$ ) contains the pseudobasis $\mathcal{B}_{M}$ (relative to $\mathrm{im}_{M}$ ), resp. $\overline{\mathcal{B}}_{M}$ (relative to $\overline{\mathrm{im}}_{M}$ ), of $U_{0}^{M^{\prime}}{ }^{*}$ dual of the PBW basis of $\mathfrak{U}_{0}^{M^{\prime}}$.

(c) $\mathcal{U}_{\varphi, \leq}^{M}$, resp. $\mathcal{U}_{\varphi, \geq}^{M}$ (hence $U_{\varphi, \leq}^{M}$, resp. $\left.U_{\varphi, \geq}^{M}\right)$ contains the pseudobasis of $U_{\varphi, \geq}^{M^{\prime} *^{*}}$, resp. of $U_{\varphi, \geq}^{M^{\prime}}{ }^{*}$, dual of the $P B W$ basis of $\mathfrak{U}_{\varphi, \leq}^{M^{\prime}}$, resp. of $\mathfrak{U}_{\varphi, \geq}^{M^{\prime}}$. The elements of this pseudobasis have form $\mathcal{F}^{\varphi} \cdot \psi$, resp. $\bar{\psi} \cdot \mathcal{E}^{\varphi}$, where $\mathcal{F}^{\varphi}$, resp. $\mathcal{E}^{\varphi}$, is an ordered monomial in the $\bar{F}_{\alpha}^{\varphi}$ 's, resp. the $\bar{E}_{\alpha}^{\varphi}$ 's, and $\psi \in \mathcal{U}_{\varphi, 0}^{M}$.

Proof. Claim (a) is trivial. As for (b) and (c), let $\mathfrak{E}_{\eta} \cdot u_{\tau}$ be any PBW monomial of $\mathfrak{U}_{\varphi, \geq}^{M^{\prime}} \cong \mathfrak{U}_{+} \otimes \mathfrak{U}_{0}^{M^{\prime}}$, with $u_{\tau}:=\prod_{i=1}^{n}\left(\begin{array}{c}\Lambda_{i} ; 0 \\ t_{i}\end{array}\right) \cdot \Lambda_{i}^{-\operatorname{Ent}\left(t_{i} / 2\right)}(\tau=$ $\left.\left(t_{1}, \ldots, t_{n}\right) \in \mathbb{N}^{n}\right)$ and $\mathfrak{E}_{\eta}:=\prod_{k=N}^{1} E_{\alpha^{k}}^{\left(e_{k}\right)}\left(\eta=\left(e_{1}, \ldots, e_{N}\right) \in \mathbb{N}^{N}\right)$. Let also $\mathcal{F}_{\phi}^{\varphi}:=\prod_{k=N}^{1}\left(\bar{F}_{\alpha^{k}}^{\varphi}\right)^{\left(f_{k}\right)} \quad\left(\phi=\left(f_{1}, \ldots, f_{N}\right) \in \mathbb{N}^{n}\right)$ be any (modified) PBW monomial of $\mathcal{U}_{\varphi,-}$. Then (for all $\mu \in M, \nu \in M^{\prime}$ )

$$
\pi_{\varphi}\left(\mathcal{F}_{\phi}^{\varphi} \cdot L_{-(1+\varphi)(\mu)}, \mathfrak{E}_{\eta} \cdot L_{\nu}\right)=c_{\eta} \cdot \delta_{\phi, \eta} \cdot q^{+(\mu \mid \nu)} \cdot q^{-\left(\mu \mid s\left(\mathfrak{E}_{\eta}\right)\right)}
$$

by $(2.3)$, where $c_{\eta}:=(-1)^{\sum_{k=1}^{N} e_{k}} \cdot q^{-\sum_{h<k}\left(e_{h} \tau_{\alpha} \mid e_{k} \alpha^{k}\right)} \cdot q^{\sum_{k=1}^{N} d_{\alpha k}\left(\begin{array}{c}e_{k} \\ 2\end{array}\right)}$ is independent of $\mu$ and $\nu$. Therefore only PBW monomials of shape $\mathcal{F}_{\phi}^{\varphi} \cdot z$ $\left(z \in U_{\varphi, 0}^{M}\right)$ give non zero values when paired with $\mathfrak{E}_{\eta} \cdot L_{\nu}$, hence also with $\mathfrak{E}_{\eta} \cdot u_{\tau}$. Now direct computation gives

$$
\begin{aligned}
& \left\langle\mathcal{F}_{\eta}^{\varphi} \cdot L_{-(1+\varphi)(\mu)}, \mathfrak{E}_{\eta} \cdot u_{\tau}\right\rangle_{\pi_{\varphi}}= \\
& =c_{\eta} \cdot q^{-\left(\mu \mid s\left(\mathfrak{E}_{\eta}\right)\right)} \cdot \prod_{i=1}^{n}\left(\begin{array}{c}
m_{i} \\
t_{i}
\end{array}\right)_{q_{i}} \cdot q^{-d_{i} m_{i} \cdot \operatorname{Ent}\left(t_{i} / 2\right)} \quad \forall \mu, \tau \in \mathbb{N}^{n}
\end{aligned}
$$

where we identify $M_{+} \cong \mathbb{N}^{n}$ so that $M_{+} \ni \mu=m_{1} \mu_{1}+\cdots+m_{n} \mu_{n} \cong$ $\left(m_{1}, \ldots, m_{n}\right) \in \mathbb{N}^{n}$. Then endowing $\mathbb{N}^{n}$ with the product ordering (of the natural ordering of $\mathbb{N}$ ) we have

$$
\begin{aligned}
& \left\langle\mathcal{F}_{\eta}^{\varphi} \cdot L_{-(1+\varphi)(\mu)}, \mathfrak{E}_{\eta} \cdot u_{\tau}\right\rangle_{\pi_{\varphi}} \neq 0 \Longleftrightarrow \tau \preceq \mu \\
& \left\langle\mathcal{F}_{\eta}^{\varphi} \cdot L_{-(1+\varphi)(\tau)}, \mathfrak{E}_{\eta} \cdot u_{\tau}\right\rangle_{\pi_{\varphi}}=c_{\eta} \cdot q^{-\left(\tau \mid s\left(\mathfrak{E}_{\eta}\right)\right)} \cdot q^{-T(\tau)} \quad \forall \tau \in \mathbb{N}^{n}
\end{aligned}
$$

where $T(\tau):=\sum_{i=1}^{n} d_{i} t_{i} \operatorname{Ent}\left(t_{i} / 2\right)$; in particular $C_{\eta, \tau}:=c_{\eta} \cdot q^{-\left(\mu \mid s\left(\mathfrak{E}_{\eta}\right)\right)}$. $q^{-T(\tau)}$ is invertible in $k\left[q, q^{-1}\right]$. Thus we have formulas (for all $\tau \in \mathbb{N}^{n}$ ) $\mathcal{F}_{\eta}^{\varphi} \cdot L_{-(1+\varphi)(\tau)}=C_{\eta, \tau} \cdot\left(\mathfrak{E}_{\eta} \cdot u_{\tau}\right)^{*}+\sum_{\tau^{\prime} \prec \tau}\left\langle\mathcal{F}_{\eta}^{\varphi} \cdot L_{-(1+\varphi)(\tau)}, \mathfrak{E}_{\eta} \cdot u_{\tau^{\prime}}\right\rangle_{\pi_{\varphi}} \cdot\left(\mathfrak{E}_{\eta} \cdot u_{\tau^{\prime}}\right)^{*}$ which tell us that $\left\{\mathcal{F}_{\eta}^{\varphi} \cdot L_{-(1+\varphi)(\tau)} \mid \tau \in \mathbb{N}^{n}\right\}$ is obtained from $\left\{\left(\mathfrak{E}_{\eta} \cdot u_{\tau}\right)^{*} \mid \tau \in\right.$ $\left.\mathbb{N}^{n}\right\}$ by means of the matrix $\mathbb{M}:=\left(\left\langle\mathcal{F}_{\eta}^{\varphi} \cdot L_{-(1+\varphi)(\tau)}, \mathfrak{E}_{\eta} \cdot u_{\tau^{\prime}}\right\rangle_{\pi_{\varphi}}\right)_{\tau, \tau^{\prime} \in \mathbb{N}^{n}}$ which has lower triangular shape, all entries in $k\left[q, q^{-1}\right]$, and diagonal entries invertible in $k\left[q, q^{-1}\right]$; then the inverse matrix $\mathbb{M}^{-1}$ has the same properties, 
whence (c) follows for $\mathcal{U}_{\varphi, \leq}^{M}$. The same proof applies for $\mathcal{U}_{\varphi, \geq}^{M}$ with $\overline{\pi_{\varphi}}$ instead of $\pi_{\varphi}$, and also gives (b) for $\eta=0$.

Remark 5.5. Since $D_{M^{\prime}} \cong U_{\geq}^{M^{\prime}} \otimes U_{\leq}^{Q} \cong U_{+} \otimes U_{0}^{M^{\prime}} \otimes U_{0}^{Q} \otimes U_{-}$, we have $D_{M^{\prime}}{ }^{*} \cong U_{\geq}^{M^{\prime *}} \widehat{\otimes} U_{\leq}^{Q^{*}} \cong U_{+}{ }^{*} \widehat{\otimes} U_{0}^{M^{\prime *}} \widehat{\otimes} U_{0}^{\widehat{Q}^{*}} \widehat{\otimes} U_{-}{ }^{*}$; hence from Lemma 5.4 we deduce that:

Every element $f \in D_{M^{\prime}}{ }^{*}$ has a unique expression as formal series

$$
f=\sum_{\mathcal{F}, \mathcal{M}, \mathcal{L}, \mathcal{E}} a_{\mathcal{F}, \mathcal{M}, \mathcal{L}, \mathcal{E}} \cdot \mathcal{F} \cdot \mathcal{M} \cdot \mathcal{L} \cdot \mathcal{E}
$$

in which $a_{\mathcal{F}, \mathcal{M}, \mathcal{L}, \mathcal{E}} \in k(q), \mathcal{M} \in \mathcal{B}_{M}, \mathcal{L} \in \overline{\mathcal{B}}_{P}$, and the $\mathcal{F}^{\varphi}$ 's, resp. the $\mathcal{E}^{\varphi}$ 's, are ordered monomials in the $F_{\alpha}^{\varphi}$ 's, resp. in the $E_{\alpha}^{\varphi}$ 's.

In particular, every $f \in D_{M^{\prime}}{ }^{*}$ can be uniquely expressed as a formal series in the $F_{\alpha^{1}}^{\varphi}, \ldots, F_{\alpha^{N}}^{\varphi}, E_{\alpha^{1}}^{\varphi}, \ldots, E_{\alpha^{N}}^{\varphi}$ with coefficients in $\left(U_{0}^{M^{\prime}} \otimes U_{0}^{Q}\right)^{*} \cong$ $U_{0}^{M^{*}} \widehat{\otimes} U_{0}^{Q *}$.

Similarly the triangular decompositions $U_{+} \otimes U_{0}^{M^{\prime}} \otimes U_{-} \cong U_{q}^{M^{\prime}}(\mathfrak{g}) \cong U_{-} \otimes$ $U_{0}^{M^{\prime}} \otimes U_{+}$give $U_{+}{ }^{*} \widehat{\otimes} U_{0}^{M^{\prime *}} \widehat{\otimes} U_{-}^{*} \cong U_{q}^{M^{\prime}}(\mathfrak{g})^{*} \cong U_{-}^{*} \widehat{\otimes} U_{0}^{M^{\prime *}} \widehat{\otimes} U_{+}{ }^{*}$, whence Lemma 5.4 implies that:

Every $f \in U_{q}^{M^{\prime}}(\mathfrak{g})^{*}$ can be uniquely expressed as a formal series in the $F_{\alpha^{1}}^{\varphi}, \ldots, F_{\alpha^{N}}^{\varphi}, E_{\alpha^{1}}^{\varphi}, \ldots, E_{\alpha^{N}}^{\varphi}$ with coefficients in $U_{0}^{M^{\prime *}}$.

In the sequel when considering the composed embedding $U_{0}^{M} \hookrightarrow U_{0}^{M^{\prime *}} \hookrightarrow$ $U_{q}^{M^{\prime}}(\mathfrak{g})^{*}$ we shall always mean that the first embedding is induced by $\overline{\pi_{\varphi}}$ (cf. (5.1)).

Proposition 5.6. The monomorphism $j_{M}: U_{q, \varphi}^{M^{\prime}}(\mathfrak{g})^{*} \hookrightarrow D_{M^{\prime}}{ }^{*} \quad(c f . \S 5.1)$ is given by

$$
j_{M}: \quad F_{i}^{\varphi} \mapsto F_{i}^{\varphi} \otimes 1, \quad L_{\mu} \mapsto L_{-(1+\varphi)(\mu)} \otimes L_{(1-\varphi)(\mu)}, \quad E_{i}^{\varphi} \mapsto 1 \otimes E_{i}^{\varphi} \forall i, \mu ;
$$

in particular the image of $j_{M}$ is the closure of the subalgebra generated by the set

$$
\left\{F_{i}^{\varphi} \otimes 1, L_{-(1+\varphi)(\mu)} \otimes L_{(1-\varphi)(\mu)}, 1 \otimes E_{i}^{\varphi} \mid i=1, \ldots, n, \mu \in M\right\} .
$$

Proof. For PBW monomials we have $p r_{M}(E \cdot L \otimes K \cdot F)=E \cdot L \cdot K \cdot F$; therefore (5.2) comes out of the definition $j_{M}:=\left(p r_{M}\right)^{*}$. As an example

$$
\begin{array}{r}
\left\langle j_{M}\left(L_{\mu}\right), E \cdot L_{\nu} \otimes K_{\alpha} \cdot F\right\rangle=\left\langle L_{\mu}, E \cdot L_{\nu} \cdot K_{\alpha} \cdot F\right\rangle_{\bar{\pi}_{\varphi}}=\delta_{E, 1} \cdot \delta_{E, 1} \cdot q^{(\mu \mid \nu+\alpha)} \\
\left\langle L_{-(1+\varphi)(\mu)} \otimes L_{(1-\varphi)(\mu)}, E \cdot L_{\nu} \otimes K_{\alpha} \cdot F\right\rangle_{\pi_{\varphi} \otimes \bar{\pi}_{\varphi}}=\delta_{E, 1} \cdot \delta_{F, 1} \cdot q^{(\mu \mid \nu+\alpha)}
\end{array}
$$


whence $j_{M}\left(L_{\mu}\right)=L_{-(1+\varphi)(\mu)} \otimes L_{(1-\varphi)(\mu)}$. Since $j_{M}:=\left(p r_{M^{\prime}}\right)^{*}$ is continuous (cf. $\S 1.1$ ), by Lemma 5.4 and Remark 5.5 it is uniquely determined by (5.2).

Remark 5.7. Now we can identify $j_{M}\left(U_{q}^{M^{\prime}}(\mathfrak{g})^{*}\right)$ with the space of formal series in the $F_{\alpha^{1}}^{\varphi}, \ldots, F_{\alpha^{N}}^{\varphi}, E_{\alpha^{1}}^{\varphi}, \ldots, E_{\alpha^{N}}^{\varphi}$ with coefficients in $j_{M}\left(U_{0}^{M^{\prime *}}\right)$. In order to locate the image - under $j_{M}$ - of the pseudobasis of $j_{M}\left(U_{q, \varphi}^{M^{\prime}}(\mathfrak{g})^{*}\right)$ dual of the PBW basis of $\mathfrak{U}_{\varphi}^{M^{\prime}}(\mathfrak{g})$, let

$$
\begin{gathered}
\mathfrak{E}_{\eta}:=\prod_{k=N}^{1} E_{\alpha^{k}}^{\left(e_{k}\right)}, \quad u_{\tau}:=\prod_{i=1}^{n}\left(\begin{array}{c}
\Lambda_{i} ; 0 \\
t_{i}
\end{array}\right) \cdot \Lambda_{i}^{-\operatorname{Ent}\left(t_{i} / 2\right)}, \quad \mathfrak{F}_{\phi}:=\prod_{k=1}^{N} F_{\alpha^{k}}^{\left(f_{k}\right)} \\
X_{\eta, \tau, \phi}:=\mathfrak{E}_{\eta} \cdot u_{\tau} \cdot \mathfrak{F}_{\phi}, \quad \mathcal{F}_{\phi}:=\prod_{k=N}^{1}\left(\bar{F}_{\alpha^{k}}^{\varphi}\right)^{f_{k}}, \quad \mathcal{E}_{\eta}:=\prod_{k=1}^{N}\left(\bar{E}_{\alpha^{k}}^{\varphi}\right)^{e_{k}}
\end{gathered}
$$

and $L_{\mu}^{\varphi, \otimes}:=L_{-(1+\varphi)(\mu)} \otimes L_{(1-\varphi)(\mu)}$. Then (2.3) gives (for some $a, b \in \mathbb{Z}$ and $\varepsilon= \pm 1$ )

$$
\begin{aligned}
& \left\langle\mathcal{F}_{\phi}^{\varphi} \cdot L_{\mu}^{\varphi, \otimes} \cdot \mathcal{E}_{\eta}^{\varphi}, \mathfrak{E}_{\bar{\eta}} \otimes u_{\tau} \cdot \mathfrak{F}_{\bar{\phi}}\right\rangle_{\pi_{\varphi} \otimes \bar{\pi}_{\varphi}}= \\
& =\delta_{\phi, \bar{\eta}} \delta_{\eta, \bar{\phi}} \cdot \varepsilon q^{a+b-\left(\mu \mid s\left(\mathfrak{E}_{\bar{\eta}}\right)\right)-\left(\mu \mid s\left(\mathfrak{F}_{\bar{\phi}}\right)\right)} \cdot \prod_{i=1}^{n}\left(\begin{array}{c}
m_{i} \\
t_{i}
\end{array}\right)_{q_{i}} q^{-d_{i} m_{i} \cdot \operatorname{Ent}\left(t_{i} / 2\right)} .
\end{aligned}
$$

Thus among the elements of the form $\mathcal{F}_{\phi}^{\varphi} \cdot L_{\mu}^{\varphi, \otimes} \cdot \mathcal{E}_{\eta}^{\varphi}$ only those with $(\phi, \eta)=(\bar{\eta}, \bar{\phi})$ and $\mu \preceq \tau$ takes non-zero value on $\mathfrak{E}_{\bar{\eta}} \otimes u_{\tau} \cdot \mathfrak{F}_{\bar{\phi}}$. Therefore

$$
\begin{aligned}
& \mathcal{F}_{\bar{\eta}}^{\varphi} \cdot L_{\tau}^{\varphi, \otimes} \cdot \mathcal{E}_{\bar{\phi}}^{\varphi}=\varepsilon q^{z} \cdot X_{\bar{\eta}, \tau, \bar{\phi}}{ }^{*}+\sum_{\tau^{\prime} \prec \tau}\left\langle\mathcal{F}_{\bar{\eta}}^{\varphi} \cdot L_{\tau}^{\varphi, \otimes} \cdot \mathcal{E}_{\bar{\phi}}^{\varphi}, X_{\bar{\eta}, \tau^{\prime}, \bar{\phi}}\right\rangle \cdot X_{\bar{\eta}, \tau^{\prime}, \bar{\phi}}{ }^{*} \\
& =\varepsilon q^{z} \cdot X_{\bar{\eta}, \tau, \bar{\phi}}^{*}+\sum_{\tau^{\prime} \prec \tau} c_{\tau, \tau^{\prime}} \cdot X_{\bar{\eta}, \tau^{\prime}, \bar{\phi}}^{*}
\end{aligned}
$$

(with $z \in \mathbb{Z}, c_{\tau, \tau^{\prime}} \in k\left[q, q^{-1}\right]$; we set also $c_{\tau, \tau}:=\varepsilon q^{z}$ and $c_{\tau, \tau^{\prime}}:=0$ for $\left.\tau^{\prime} \nprec \tau\right)$; then we turn from $\left\{X_{\bar{\eta}, \tau^{\prime}, \bar{\phi}}{ }^{*} \mid \tau^{\prime} \in \mathbb{N}^{n}\right\}$ to $\left\{\mathcal{F}_{\bar{\eta}}^{\varphi} \cdot L_{\tau}^{\varphi, \otimes} \cdot \mathcal{E}_{\bar{\phi}}^{\varphi} \mid \tau \in \mathbb{N}^{n}\right\}$ by means of a lower triangular matrix $\mathbb{M}_{\bar{\eta}, \bar{\phi}}:=\left(c_{\tau, \tau^{\prime}}\right)_{\tau, \tau^{\prime} \in \mathbb{N}^{n}}$, whose entries belong to $k\left[q, q^{-1}\right]$ and whose diagonal entries are invertible in $k\left[q, q^{-1}\right]$; then letting $\left(\mathbb{M}_{\bar{\eta}, \bar{\phi}}\right)^{-1}=\left(c_{\tau, \tau^{\prime}}^{\prime}\right)_{\tau, \tau^{\prime} \in \mathbb{N}^{n}}$ we find that $X_{\eta, \tau, \phi}{ }^{*}=\sum_{\tau^{\prime} \preceq \tau} c_{\tau, \tau^{\prime}}^{\prime}$. $\mathcal{F}_{\bar{\eta}}^{\varphi} \cdot L_{\tau^{\prime}}^{\varphi, \otimes} \cdot \mathcal{E}_{\bar{\phi}}^{\varphi}$. Now let $B_{\bar{\eta}, \tau, \bar{\phi}}^{\varphi, \otimes}:=\sum_{\tau^{\prime} \preceq \tau} c_{\tau, \tau^{\prime}}^{\prime} \cdot L_{\tau^{\prime}}^{\varphi, \otimes}:$ then

$$
X_{\bar{\eta}, \tau, \bar{\phi}}{ }^{*}=\mathcal{F}_{\bar{\eta}}^{\varphi} \cdot B_{\bar{\eta}, \tau, \bar{\phi}}^{\varphi, \otimes} \cdot \mathcal{E}_{\bar{\phi}}^{\varphi}
$$


thus $\left\{\mathcal{F}_{\bar{\eta}}^{\varphi} \cdot B_{\bar{\eta}, \tau, \bar{\phi}}^{\varphi, \otimes} \cdot \mathcal{E}_{\bar{\phi}}^{\varphi} \mid \bar{\eta} \in \mathbb{N}^{N}, \tau \in \mathbb{N}^{n}, \bar{\phi} \in \mathbb{N}^{N}\right\}$ is the image pseudobasis (of $j_{M}\left(U_{q, \varphi}^{M^{\prime}}(\mathfrak{g})\right)$ ) we were looking for; in particular we stress the fact that:

The pseudobasis of $j_{M}\left(U_{q, \varphi}^{M^{\prime}}(\mathfrak{g})^{*}\right)$ dual of the PBW basis of $\mathfrak{U}_{\varphi}^{M^{\prime}}(\mathfrak{g})$ is contained in $j_{M}\left(U_{q, \varphi}^{M^{\prime}}(\mathfrak{g})^{*}\right) \cap\left(\mathcal{U}_{\varphi,-} \otimes \mathcal{U}_{\varphi, 0}^{M} \otimes \mathcal{U}_{\varphi,+}\right)$.

5.8. Integer forms. We want to study the subspaces of linear functions on $U_{q, \varphi}^{M^{\prime}}(\mathfrak{g})$ which are "integer-valued" on its integer forms. Thus we define

$$
\begin{aligned}
\mathfrak{U}_{\varphi}^{M^{\prime}}(\mathfrak{g})^{*}:=\left\{f \in U_{q, \varphi}^{M^{\prime}}(\mathfrak{g})^{*} \mid\left\langle f, \mathfrak{U}_{\varphi}^{M^{\prime}}(\mathfrak{g})\right\rangle \subseteq k\left[q, q^{-1}\right]\right\} \\
\mathcal{U}_{\varphi}^{M^{\prime}}(\mathfrak{g})^{*}:=\left\{f \in U_{q, \varphi}^{M^{\prime}}(\mathfrak{g})^{*} \mid\left\langle f, \mathcal{U}_{\varphi}^{M^{\prime}}(\mathfrak{g})\right\rangle \subseteq k\left[q, q^{-1}\right]\right\} \\
\mathfrak{I}_{\varphi}^{M}:=\left\{f \in j_{M}\left(U_{q, \varphi}^{M^{\prime}}(\mathfrak{g})^{*}\right) \mid\left\langle f, \mathfrak{U}_{\varphi}^{M^{\prime}}(\mathfrak{g})\right\rangle \subseteq k\left[q, q^{-1}\right]\right\} \\
\mathcal{I}_{\varphi}^{M}:=\left\{f \in j_{M}\left(U_{q, \varphi}^{M^{\prime}}(\mathfrak{g})^{*}\right) \mid\left\langle f, \mathcal{U}_{\varphi}^{M^{\prime}}(\mathfrak{g})\right\rangle \subseteq k\left[q, q^{-1}\right]\right\} \\
\mathfrak{U}_{\varphi, 0}^{M^{\prime}}{ }^{*}:=\left\{f \in U_{\varphi, 0}^{M^{\prime}} \mid\left\langle f, \mathfrak{U}_{\varphi, 0}^{M^{\prime}}\right\rangle \subseteq k\left[q, q^{-1}\right]\right\} \\
\mathcal{U}_{\varphi, 0}^{M^{\prime}}{ }^{*}:=\left\{f \in U_{\varphi, 0}^{M^{\prime}} \mid\left\langle f, \mathcal{U}_{\varphi, 0}^{M^{\prime}}\right\rangle \subseteq k\left[q, q^{-1}\right]\right\}
\end{aligned}
$$

notice that $j_{M}$ restricts to isomorphisms $j_{M}: \mathfrak{U}_{\varphi}^{M^{\prime}}(\mathfrak{g})^{*} \stackrel{\cong}{\longrightarrow} \mathfrak{I}_{\varphi}^{M}$ and $j_{M}: \mathcal{U}_{\varphi}^{M^{\prime}}(\mathfrak{g})^{*} \stackrel{\cong}{\longrightarrow} \mathcal{I}_{\varphi}^{M}$.

Proposition 5.9. (a) $\mathfrak{U}_{\varphi}^{M^{\prime}}(\mathfrak{g})^{*}$ is the $k\left[q, q^{-1}\right]$-submodule $\left(\right.$ of $\left.U_{q, \varphi}^{M^{\prime}}(\mathfrak{g})^{*}\right)$ of formal series $(c f . \S 5.5) \quad \sum_{\mathcal{F} \varphi, \psi, \mathcal{E}^{\varphi}} \mathcal{F}^{\varphi} \cdot \psi \cdot \mathcal{E}^{\varphi}$ in which $\psi \in \mathfrak{U}_{\varphi, 0}^{M^{\prime *}}$ and the $\mathcal{F}^{\varphi}$ 's, resp. the $\mathcal{E}^{\varphi}$ 's, are monomials of the $P B W$ basis of $\mathcal{U}_{\varphi,-}$, resp. of $\mathcal{U}_{\varphi,+}$.

In particular $\mathfrak{U}_{\varphi}^{M^{\prime}}(\mathfrak{g})^{*}$ is a formal Hopf subalgebra of $U_{q, \varphi}^{M^{\prime}}(\mathfrak{g})^{*}$.

(b) $\mathcal{U}_{\varphi}^{M^{\prime}}(\mathfrak{g})^{*}$ is the $k\left[q, q^{-1}\right]$-submodule (of $U_{q, \varphi}^{M^{\prime}}(\mathfrak{g})^{*}$ ) of formal series (cf. §5.5) $\sum_{\mathfrak{F}^{\varphi}, \phi, \mathfrak{E} \varphi} \mathfrak{F}^{\varphi} \cdot \phi \cdot \mathfrak{E}^{\varphi}$ in which $\phi \in \mathcal{U}_{\varphi, 0}^{M^{\prime *}}$ and the $\mathfrak{F}^{\varphi}$ 's, resp. the $\mathfrak{E}^{\varphi}$ 's, are monomials of the PBW basis of $\mathfrak{U}_{\varphi,-}$, resp. of $\mathfrak{U}_{\varphi,+}$.

In particular $\mathcal{U}_{\varphi}^{M^{\prime}}(\mathfrak{g})^{*}$ is a formal Hopf subalgebra of $U_{q, \varphi}^{M^{\prime}}(\mathfrak{g})^{*}$.

Proof. Let us prove (b). Let $f \in U_{q, \varphi}^{M^{\prime}}(\mathfrak{g})^{*}$ be given, and expand it as a series $f=\sum_{\phi, \eta \in \mathbb{N}^{N}} \mathfrak{F}_{\phi}^{\varphi} \cdot \Phi_{\phi, \eta}^{\varphi} \cdot \mathfrak{E}_{\eta}^{\varphi}$ in which the $\mathfrak{F}_{\phi}^{\varphi}$,s, resp. the $\mathfrak{E}_{\eta}^{\varphi}$ 's, are PBW monomials of $\mathfrak{U}_{\varphi,-}$, resp. of $\mathfrak{U}_{\varphi,+}$, and $\Phi_{\phi, \eta}^{\varphi} \in U_{\varphi, 0}^{M^{\prime *}}$. Let $\Phi_{\phi, \eta}^{\varphi}=\sum_{\tau \in \mathbb{N}^{n}} a_{\phi, \eta}^{\tau} B_{\phi, \tau, \eta}^{\varphi}$ and $j_{M}\left(B_{\phi, \tau, \eta}^{\varphi}\right)=\sum_{\mu \preceq \tau} c_{\tau}^{\mu} \cdot L_{\mu}^{\varphi, \otimes}=B_{\phi, \tau, \eta}^{\varphi, \otimes}$. For all monomials $\mathcal{E}_{\bar{\eta}} \cdot L_{\nu} \cdot \mathcal{F}_{\bar{\phi}}$ of a PBW basis of $\mathcal{U}_{\varphi}^{M^{\prime}}(\mathfrak{g})$ we have

$$
\begin{aligned}
& \left\langle f, \mathcal{E}_{\bar{\eta}} \cdot L_{\nu} \cdot \mathcal{F}_{\bar{\phi}}\right\rangle=\sum_{\phi, \tau, \eta} a_{\phi, \eta}^{\tau} \cdot\left\langle\mathfrak{F}_{\phi}^{\varphi} \cdot B_{\tau}^{\varphi} \cdot \mathfrak{E}_{\eta}^{\varphi}, \mathcal{E}_{\bar{\eta}} \cdot L_{\nu} \cdot \mathcal{F}_{\bar{\phi}}\right\rangle \\
& =\sum_{\phi, \tau, \eta} a_{\phi, \eta}^{\tau} \cdot \sum_{\mu \preceq \tau} c_{\tau}^{\mu} \cdot\left\langle\mathfrak{F}_{\phi}^{\varphi} \cdot L_{-(1+\varphi)(\mu)}, \mathcal{E}_{\bar{\eta}} \cdot L_{\nu}\right\rangle_{\pi_{\varphi}} \cdot\left\langle L_{(1-\varphi)(\mu)} \cdot \mathfrak{E}_{\eta}^{\varphi}, \mathcal{F}_{\bar{\phi}}\right\rangle_{\overline{\pi_{\varphi}}}
\end{aligned}
$$




$$
\begin{aligned}
& = \pm q^{a+b} \cdot \sum_{\tau} a_{\bar{\eta}, \bar{\phi}}^{\tau} \cdot\left\langle B_{\tau}^{\varphi}, L_{\nu-\left(s\left(\mathfrak{E}_{\bar{\phi}}\right)+s\left(\mathfrak{F}_{\bar{\eta}}\right)\right)}\right\rangle \\
& = \pm q^{a+b} \cdot\left\langle\Phi_{\bar{\eta}, \bar{\phi}}^{\varphi}, L_{\nu-\left(s\left(\mathfrak{E}_{\bar{\phi}}\right)+s\left(\mathfrak{F}_{\bar{\eta}}\right)\right)}\right\rangle
\end{aligned}
$$

for some $a, b \in \mathbb{Z}$ depending only respectively on $\bar{\eta}$ and $\bar{\phi}$. Then if $\Phi_{\bar{\eta}, \bar{\phi}}^{\varphi} \in$ $\mathcal{U}_{\varphi, 0}^{M^{\prime *}}$ we have $\left\langle f, \mathcal{E}_{\bar{\eta}} \cdot L_{\nu} \cdot \mathcal{F}_{\bar{\phi}}\right\rangle \in k\left[q, q^{-1}\right]$ for all $\bar{\eta}, \nu, \bar{\phi}$, hence $f \in$ $\mathcal{U}_{\varphi}^{M^{\prime}}(\mathfrak{g})^{*} ;$ conversely, the latter gives $\left\langle\Phi_{\bar{\eta}, \bar{\phi}}^{\varphi}, L_{\nu^{\prime}}\right\rangle \in k\left[q, q^{-1}\right]$ for all $\nu^{\prime} \in M^{\prime}$, hence $\Phi_{\bar{\eta}, \bar{\phi}}^{\varphi} \in \mathcal{U}_{\varphi, 0}^{M^{* *}}$.

Now consider the Hopf structure. Let $f \in \mathcal{U}_{\varphi}^{M^{\prime}}(\mathfrak{g})^{*}$, and expand $\Delta(f)$ as a series $\Delta(f)=\sum_{\sigma}\left(\mathfrak{F}_{\sigma}^{\varphi} \cdot \phi_{\sigma} \cdot \mathfrak{E}_{\sigma}^{\varphi}\right) \otimes\left(\mathfrak{F}_{\sigma}^{\varphi^{\prime}} \cdot \phi_{\sigma}^{\prime} \cdot \mathfrak{E}_{\sigma}^{\varphi^{\prime}}\right)$ so that $\phi_{\sigma} \otimes \phi_{\sigma}^{\prime} \neq \phi_{\tau} \otimes \phi_{\tau}^{\prime}$ for all $\sigma, \tau$, such that $\left(\mathfrak{F}_{\sigma}^{\varphi}, \mathfrak{E}_{\sigma}^{\varphi}, \mathfrak{F}_{\sigma}^{\varphi^{\prime}}, \mathfrak{E}_{\sigma}^{\varphi^{\prime}}\right) \neq\left(\mathfrak{F}_{\tau}^{\varphi}, \mathfrak{E}_{\tau}^{\varphi}, \mathfrak{F}_{\tau}^{\varphi^{\prime}}, \mathfrak{E}_{\tau}^{\varphi^{\prime}}\right)$ (this is always possible). As $f \in \mathcal{U}_{\varphi}^{M^{\prime}}(\mathfrak{g})$, then $\Delta(f)$ is integer-valued on $\mathcal{U}_{\varphi}^{M^{\prime}}(\mathfrak{g}) \otimes \mathcal{U}_{\varphi}^{M^{\prime}}(\mathfrak{g})$. Fix any $\bar{\sigma}$ : Exploiting (2.3) we get the existence of unique (non-modified) PBW monomials $\mathcal{E}_{\bar{\sigma}}, \mathcal{F}_{\bar{\sigma}}, \mathcal{E}_{\bar{\sigma}}^{\prime}, \mathcal{F}_{\bar{\sigma}}^{\prime}$ such that

$\left\langle\Delta(f),\left(\mathcal{E}_{\bar{\sigma}} \otimes \mathcal{E}_{\bar{\sigma}}^{\prime}\right) \cdot\left(L_{\nu} \otimes L_{\nu^{\prime}}\right) \cdot\left(\mathcal{F}_{\bar{\sigma}} \otimes \mathcal{F}_{\bar{\sigma}}^{\prime}\right)\right\rangle= \pm q^{c_{\bar{\sigma}}} \cdot\left\langle\phi_{\sigma} \otimes \phi_{\sigma}^{\prime}, L_{\nu+\xi} \otimes L_{\nu^{\prime}+\xi^{\prime}}\right\rangle$ for all $\nu, \nu^{\prime} \in M^{\prime}$ (for some $c_{\bar{\sigma}} \in \mathbb{Z}$ and $\xi, \xi^{\prime} \in Q\left(\subseteq M^{\prime}\right)$ independent of $\nu$, $\left.\nu^{\prime}\right)$; since $\Delta(f)$ is integer-valued, $\phi_{\bar{\sigma}} \otimes \phi_{\bar{\sigma}}^{\prime}$ is integer-valued on $\mathcal{U}_{\varphi, 0}^{M^{\prime}} \otimes \mathcal{U}_{\varphi, 0}^{M^{\prime}}$, that is $\phi_{\bar{\sigma}} \otimes \phi_{\bar{\sigma}}^{\prime} \in\left(\mathcal{U}_{\varphi, 0}^{M^{\prime}} \otimes \mathcal{U}_{\varphi, 0}^{M^{\prime}}\right)^{*}=\mathcal{U}_{\varphi, 0}^{M^{\prime}} \widehat{\otimes}^{*} \mathcal{U}_{\varphi, 0}^{M^{\prime *}}$; but $\phi_{\bar{\sigma}} \otimes \phi_{\bar{\sigma}}^{\prime} \in U_{\varphi, 0}^{M^{\prime *}} \otimes U_{\varphi, 0}^{M^{\prime *}}$, thus $\phi_{\bar{\sigma}}, \phi_{\bar{\sigma}}^{\prime} \in \mathcal{U}_{\varphi, 0}^{M^{\prime *}}$.

Finally, we have $1 \in \mathcal{U}_{\varphi}^{M^{\prime}}(\mathfrak{g})^{*}$, because $1:=\epsilon, \epsilon\left(\mathcal{U}_{\varphi}^{M^{\prime}}(\mathfrak{g})^{*}\right) \subseteq k\left[q, q^{-1}\right]$ because $\epsilon:=1^{*}$ and $1 \in \mathcal{U}_{\varphi}^{M^{\prime}}(\mathfrak{g})$, and $S\left(\mathcal{U}_{\varphi}^{M^{\prime}}(\mathfrak{g})^{*}\right)=\mathcal{U}_{\varphi}^{M^{\prime}}(\mathfrak{g})^{*}$ because $S:=$ $S^{*}$ and $S\left(\mathcal{U}_{\varphi}^{M^{\prime}}(\mathfrak{g})\right)=\mathcal{U}_{\varphi}^{M^{\prime}}(\mathfrak{g})$. Thus $\mathcal{U}_{\varphi}^{M^{\prime}}(\mathfrak{g})^{*}$ is a formal Hopf subalgebra of $U_{q, \varphi}^{M^{\prime}}(\mathfrak{g})^{*}$, q.e.d.

Definition 5.10. We call $A_{\varphi}^{M}$ the subalgebra of $U_{\varphi, \leq}^{M} \otimes U_{\varphi, \geq}^{P}\left(\subset D_{\varphi}^{M^{\prime *}}\right)$ generated by $\left\{F_{i}^{\varphi} \otimes 1, L_{\mu}^{\varphi, \otimes}, 1 \otimes E_{i}^{\varphi} \mid i=1, \ldots, n ; \mu \in M\right\}$. Then we set

$$
\begin{aligned}
& \mathfrak{A}_{\varphi}^{M}:=\left\{f \in A_{\varphi}^{M} \mid\left\langle f, \mathfrak{U}_{\varphi}^{M^{\prime}}(\mathfrak{g})\right\rangle \subseteq k\left[q, q^{-1}\right]\right\}=A_{\varphi}^{M} \cap \mathfrak{I}_{\varphi}^{M} \\
& \mathcal{A}_{\varphi}^{M}:=\left\{f \in A_{\varphi}^{M} \mid\left\langle f, \mathcal{U}_{\varphi}^{M^{\prime}}(\mathfrak{g})\right\rangle \subseteq k\left[q, q^{-1}\right]\right\}=A_{\varphi}^{M} \cap \mathcal{I}_{\varphi}^{M} .
\end{aligned}
$$

Lemma 5.11. (a) $\mathfrak{A}_{\varphi}^{M}$ is a $k\left[q, q^{-1}\right]$-integer form of $A_{\varphi}^{M}$, generated as a $k\left[q, q^{-1}\right]$-subalgebra by

$$
\left\{\bar{F}_{\alpha^{h}}^{\varphi} \otimes 1, L_{\mu}^{\varphi, \otimes}, 1 \otimes \bar{E}_{\alpha^{k}}^{\varphi} \mid h, k=1, \ldots, N ; \mu \in M\right\} .
$$

(b) $\mathcal{A}_{\varphi}^{M}$ is a $k\left[q, q^{-1}\right]$-integer form of $A_{\varphi}^{M}$, generated as a $k\left[q, q^{-1}\right]$ subalgebra by 


$$
\begin{aligned}
\left\{\left(F_{\alpha^{h}}^{\varphi}\right)^{(a)} \otimes 1,\left(\begin{array}{c}
L_{\mu_{i}}^{\varphi, \otimes} ; c \\
t
\end{array}\right), L_{-\mu_{i}}^{\varphi, \otimes}, 1 \otimes\left(E_{\alpha^{k}}^{\varphi}\right)^{(d)} \mid\right. & \\
& \mid h, k, i=1, \ldots, n ; a, t, d \in \mathbb{N} ; c \in \mathbb{Z}\} .
\end{aligned}
$$

Proof. Definitions yield a linear isomorphism $\Phi_{M}: A_{\varphi}^{M} \cong U_{\varphi,-} \otimes U_{\varphi, 0}^{M} \otimes U_{\varphi,+}$ given by $\Phi_{M}: F_{i}^{\varphi} \otimes 1 \mapsto F_{i}^{\varphi} \otimes 1 \otimes 1, L_{\mu}^{\varphi, \otimes} \mapsto 1 \otimes L_{\mu} \otimes 1,1 \otimes E_{i}^{\varphi} \mapsto 1 \otimes 1 \otimes E_{i}^{\varphi}$; but this restricts to $\Phi: \mathcal{A}_{\varphi}^{M} \cong \mathcal{U}_{\varphi,-} \otimes \mathcal{U}_{\varphi, 0}^{M} \otimes \mathcal{U}_{\varphi,+}, \Phi: \mathfrak{A}_{\varphi}^{M} \cong \mathfrak{U}_{\varphi,-} \otimes \mathfrak{U}_{\varphi, 0}^{M} \otimes$ $\mathfrak{U}_{\varphi,+}$, so $\S 3.4$ gives the claim.

The following result stems from [CV-2], Lemma 2.5 (which extends [DL], Lemma 4.3), relating our quantum formal groups to quantum function algebras; in particular we prove that $\mathfrak{F}_{\varphi}^{M}[G]$ and $\mathcal{F}_{\varphi}^{M}[G]$ are integer forms (over $\left.k\left[q, q^{-1}\right]\right)$ of $F_{q, \varphi}^{M}[G]$ as Hopf algebras.

Proposition 5.12. (a) The monomorphism of formal Hopf algebras $j_{M}$ : $U_{q, \varphi}^{M^{\prime}}(\mathfrak{g})^{*} \longleftrightarrow D_{\varphi}^{M^{\prime *}}$ restricts to an embedding $\mu_{M}: F_{q, \varphi}^{M}[G] \longleftrightarrow D_{\varphi}^{M^{\prime *}}$ whose image is contained in $A_{\varphi}^{M}$.

(b) The embedding in (a) preserves integer forms, namely $\mathfrak{F}_{\varphi}^{M}[G]=$ $\mu_{M}{ }^{-1}\left(\mathfrak{A}_{\varphi}^{M}\right), \mathcal{F}_{\varphi}^{M}[G]=\mu_{M}{ }^{-1}\left(\mathcal{A}_{\varphi}^{M}\right)$, so that restriction provides embeddings of $k\left[q, q^{-1}\right]$-algebras $\mu_{M}: \mathfrak{F}_{\varphi}^{M}[G] \longrightarrow \mathfrak{A}_{\varphi}^{M}, \mu_{M}: \mathcal{F}_{\varphi}^{M}[G] \longleftrightarrow \mathcal{A}_{\varphi}^{M}$. It follows that

$\mathfrak{F}_{\varphi}^{M}[G]$ is a Hopf subalgebra of $F_{q, \varphi}^{M}[G]$, and a $k\left[q, q^{-1}\right]$-integer form of it, $\mathcal{F}_{\varphi}^{M}[G]$ is a Hopf subalgebra of $F_{q, \varphi}^{M}[G]$, and a $k\left[q, q^{-1}\right]$-integer form of it.

Proof. (a) The first part is obvious. As for the second, recall that the identification $D_{M^{\prime}}=U_{\varphi, \geq}^{M^{\prime}} \otimes U_{\varphi, \leq}^{Q}$ is given by $U_{\varphi, \geq}^{M^{\prime}} \otimes U_{\varphi, \leq}^{Q} \stackrel{j_{+} \otimes j_{-}}{\longrightarrow} D_{M^{\prime}} \otimes$ $D_{M^{\prime}} \stackrel{m_{D}}{\longrightarrow} D_{M^{\prime}}$ where $j_{+}: U_{\varphi, \geq}^{M^{\prime}} \hookrightarrow D_{M^{\prime}}$ and $j_{-}: U_{\varphi, \leq}^{Q} \hookrightarrow D_{M^{\prime}}$ are the natural Hopf algebra embeddings, $m_{D}$ is the multiplication of $D_{M^{\prime}}$, and we look this composition as a Hopf algebra isomorfism; then the identification $D_{M^{\prime}}{ }^{*}=U_{\varphi, \geq}^{M^{\prime}} \widehat{\otimes} U_{\varphi, \leq}^{Q}{ }^{*}$ is given by $\left(m_{D} \circ\left(j_{+} \otimes j_{-}\right)\right)^{*}=\left(j_{+}^{*} \widehat{\otimes} j_{-}^{*}\right) \circ m_{D}^{*}$. If $m_{U}$ is the multiplication of $U_{q, \varphi}^{M^{\prime}}(\mathfrak{g})$, we have $m_{U} \circ\left(p r_{M^{\prime}} \otimes p r_{M^{\prime}}\right)=p r_{M^{\prime}} \circ m_{D}$, hence dualizing yields $\left(p r_{M^{\prime}} \circ m_{D} \circ\left(j_{+} \otimes j_{-}\right)\right)^{*}=\left(p r_{M^{\prime}} \circ j_{+}\right)^{*} \widehat{\otimes}$ $\left(p r_{M^{\prime}} \circ j_{-}\right)^{*} \circ m_{U}^{*}$; but $p r_{M^{\prime}} \circ j_{ \pm}=i_{ \pm}: U_{q, \varphi}^{M^{\prime}}\left(\mathfrak{b}_{ \pm}\right) \longleftrightarrow U_{q}^{M^{\prime}}(\mathfrak{g})$ (the natural embedding), thus $\left(p r_{M^{\prime}} \circ m_{U} \circ\left(j_{+} \otimes j_{-}\right)\right)^{*}=\left(i_{+}^{*} \widehat{\otimes} i_{-}^{*}\right) \circ m_{U}^{*}$. Now $m_{U}^{*}$ is the comultiplication $\Delta$ of $U_{q, \varphi}^{M^{\prime}}(\mathfrak{g})^{*}$, which restricts to $F_{q, \varphi}^{M}[G]$, while $\rho_{ \pm}:=$ $i_{ \pm}^{*}: U_{q, \varphi}^{M^{\prime}}(\mathfrak{g})^{*} \rightarrow U_{q, \varphi}^{M^{\prime}}\left(\mathfrak{b}_{ \pm}\right)^{*}$ is the "restriction" map, which maps $F_{q, \varphi}^{M}[G]$ onto 
$F_{q, \varphi}^{M}[B] \pm$; using also (4.1), we obtain

$\left(p r_{M^{\prime}} \circ m_{U} \circ\left(j_{+} \otimes j_{-}\right)\right)^{*}\left(F_{q, \varphi}^{M}[G]\right)=\left(\rho_{+} \widehat{\otimes} \rho_{-}\right)\left(\Delta\left(F_{q, \varphi}^{M}[G]\right)\right) \subseteq U_{\varphi, \leq}^{M} \otimes U_{\varphi, \geq}^{M} ;$ in other words, $j_{M}$ maps $F_{q, \varphi}^{M}[G]$ into $U_{\varphi, \leq}^{M} \otimes U_{\varphi, \geq}^{P}$. From the very definition we get that $\mu_{M}\left(F_{q, \varphi}^{M}[G]\right)$ vanishes on $\mathfrak{K}_{M^{\prime}}^{\varphi}$, hence - by Proposition 5.6 $\mu_{M}\left(F_{q, \varphi}^{M}[G]\right) \subseteq A_{M}$, q.e.d.

(b) The first two claims are obvious by definition. Let now, for instance, $f \in \mathcal{F}_{\varphi}^{M}[G]$ : Then $\mu_{M}(S(f)) \in A_{\varphi}^{M}$ by $(\mathrm{a}), \mu_{M}(S(f))=S\left(\mu_{M}(f)\right)$, and $\left\langle S\left(\mu_{M}(f)\right), \mathcal{U}_{\varphi}^{M^{\prime}}(\mathfrak{g})\right\rangle=\left\langle\mu_{M}(f), S\left(\mathcal{U}_{\varphi}^{M^{\prime}}(\mathfrak{g})\right)\right\rangle=\left\langle\mu_{M}(f), \mathcal{U}_{\varphi}^{M^{\prime}}(\mathfrak{g})\right\rangle \subseteq$ $k\left[q, q^{-1}\right]$, hence $\mu_{M}(S(f)) \in \mathfrak{A}_{\varphi}^{M}$, thus $S(f) \in \mathcal{F}_{\varphi}^{M}[G]$; similarly, $\Delta\left(\mu_{M}(f)\right) \in A_{\varphi}^{M} \otimes A_{\varphi}^{M}$, and $\left\langle\Delta\left(\mu_{M}(f)\right), \mathcal{U}_{\varphi}^{M^{\prime}}(\mathfrak{g}) \otimes \mathcal{U}_{\varphi}^{M^{\prime}}(\mathfrak{g})\right\rangle \subseteq k\left[q, q^{-1}\right]$, hence $\left(\mu_{M} \otimes \mu_{M}\right)(\Delta(f)) \in \mathfrak{A}_{\varphi}^{M} \otimes \mathfrak{A}_{\varphi}^{M}$ : We have only to remark that

$$
\begin{aligned}
& \left(A_{\varphi}^{M} \otimes A_{\varphi}^{M}\right) \cap\left\{\phi \in j_{M}\left(\left(\left(U_{q, \varphi}^{M^{\prime}}(\mathfrak{g})\right)^{\otimes 2}\right)^{*}\right) \mid\left\langle\phi,\left(\mathcal{U}_{\varphi}^{M^{\prime}}(\mathfrak{g})\right)^{\otimes 2}\right\rangle \subseteq k\left[q, q^{-1}\right]\right\} \\
& =\mathcal{A}_{\varphi}^{M} \otimes \mathcal{A}_{\varphi}^{M} ;
\end{aligned}
$$

we conclude that $\Delta(f) \in \mathcal{F}_{\varphi}^{M}[G] \otimes \mathcal{F}_{\varphi}^{M}[G]$. Therefore $\mathcal{F}_{\varphi}^{M}[G]$ is a $k\left[q, q^{-1}\right]$ Hopf subalgebra of $F_{q, \varphi}^{M}[G]$. Finally, let $f \in F_{q, \varphi}^{M}[G]$; then $\mu_{M}(c(q) f)=$ $c(q) \cdot \mu_{M}(f) \in \mathcal{A}_{\varphi}^{M}$ for some $c(q) \in k\left[q, q^{-1}\right]$. Thus $c(q) f \in \mu_{M}{ }^{-1}\left(\mathcal{A}_{\varphi}^{M}\right)=$ $\mathcal{F}_{\varphi}^{M}[G]$, and $f=\frac{1}{c(q)} \cdot(c(q) f)$ with $c(q) f \in \mathfrak{F}_{\varphi}^{M}[G]:$ Hence $k(q) \otimes_{k\left[q, q^{-1}\right]}$ $\mathfrak{F}_{\varphi}^{M}[G]=F_{q, \varphi}^{M}[G]$, i.e., $\mathfrak{F}_{\varphi}^{M}[G]$ is $k\left[q, q^{-1}\right]$-integer form of $F_{q, \varphi}^{M}[G]$, q.e.d. The same procedure works for $\mathcal{F}_{\varphi}^{M}[G]$ too, so the proof is complete.

5.13. Matrix coefficients. The result above can be refined, extending embeddings to isomorphisms. Let $\mu \in M_{+}:=M \cap P_{+}$, and let $V_{-\mu}$ be an irreducible $U_{\varphi, 0}^{M^{\prime}}$-module of lowest weight $-\mu$ (recall that $U_{q, \varphi}^{M^{\prime}}(\mathfrak{g}) \cong U_{q, 0}^{M^{\prime}}(\mathfrak{g})$ as algebras, hence their representation theory is the same). Let $v_{-\mu} \neq 0$ be a lowest weight vector of $V_{-\mu}$, and let $\phi_{-\mu} \in V_{-\mu}{ }^{*}$ be the linear functional on $V_{-\mu}$ defined by (a) $\phi_{-\mu}\left(v_{-\mu}\right)=1$ and (b) $\phi_{-\mu}$ vanishes on the unique $U_{\varphi, 0}^{M^{\prime}}$-invariant complement of $k(q) \cdot v_{-\mu}$ in $V_{-\mu}$; let $\psi_{-\mu}:=c_{\phi_{-\mu}, v_{-\mu}}$ be the corresponding matrix coefficient, i.e., $\psi_{-\mu}: x \mapsto \phi_{-\mu}\left(x . v_{-\mu}\right)$ for all $x \in$ $U_{q, \varphi}^{M^{\prime}}(\mathfrak{g})$. The following refines Proposition 5.12, improving [DL], Theorem 4.6, and $[\mathbf{C V - 2}]$, Lemma 2.5:

Theorem 5.14. Let $\rho:=\sum_{i=1}^{n} \mu_{i}\left(\left\{\mu_{1}, \ldots, \mu_{n}\right\}\right.$ being our fixed $\mathbb{Z}$-basis of $M, c f . \S 1.1)$.

The algebra monomorphisms $\mu_{M}: F_{q, \varphi}^{M}[G] \longrightarrow A_{M}, \mu_{M}: \mathfrak{F}_{\varphi}^{M}[G]$ $\mathfrak{A}_{\varphi}^{M}$ and $\mu_{M}: \mathcal{F}_{\varphi}^{M}[G] \longleftrightarrow \mathcal{A}_{\varphi}^{M}$ respectively extend to algebra isomorphisms

$$
\mu_{M}: F_{q, \varphi}^{M}[G]\left[\psi_{-\rho}^{-1}\right] \stackrel{\cong}{\longrightarrow} A_{\varphi}^{M}, \quad \mu_{M}: \mathfrak{F}_{\varphi}^{M}[G]\left[\psi_{-\rho}^{-1}\right] \stackrel{\cong}{\longrightarrow} \mathfrak{A}_{\varphi}^{M},
$$




$$
\mu_{M}: \mathcal{F}_{\varphi}^{M}[G]\left[\psi_{-\rho}^{-1}\right] \stackrel{\cong}{\longrightarrow} \mathcal{A}_{\varphi}^{M}
$$

moreover, $\mu_{M}\left(F_{q, \varphi}^{M}[G]\right)$ and $A_{\varphi}^{M}$, resp. $\mu_{M}\left(\mathfrak{F}_{\varphi}^{M}[G]\right)$ and $\mathfrak{A}_{\varphi}^{M}$, resp. $\mu_{M}\left(\mathcal{F}_{\varphi}^{M}[G]\right)$ and $\mathcal{A}_{\varphi}^{M}$, are dense in $j_{M}\left(U_{q, \varphi}^{M^{\prime}}(\mathfrak{g})^{*}\right)$, resp. $\mathfrak{I}_{\varphi}^{M}$, resp. $\mathcal{I}_{\varphi}^{M}$.

Proof. It is proved in $[\mathrm{DL}]$, Theorem 4.6, that $\mu_{P}: \mathfrak{F}_{0}^{P}[G] \longleftrightarrow \mathfrak{A}_{0}^{P}$ extends to an isomorphism of $k\left[q, q^{-1}\right]$-algebras $\mu_{P}: \mathfrak{F}_{0}^{P}[G]\left[\psi_{-\rho}^{-1}\right] \stackrel{\cong}{\longrightarrow} \mathfrak{A}_{0}^{P}:$ in particular scalar extension gives $\mu_{P}: F_{q, 0}^{P}[G]\left[\psi_{-\rho}^{-1}\right] \stackrel{\cong}{\longrightarrow} A_{0}^{P}$. This is easily extended to general $\varphi$ and $M$.

Now, computations like in [DL] give also $\mu_{M}\left(\psi_{-\mu}\right)=L_{-\mu}^{\varphi, \otimes}$ for all $\mu \in$ $M_{+}$; therefore $\mu_{M}\left(\psi_{-\rho}^{-1}\right)=L_{\rho}^{\varphi, \otimes}$. Again from the proof in [DL] we get $F_{i}^{\varphi} L_{-\mu_{i}}^{\varphi, \otimes}, L_{-\mu_{i}}^{\varphi, \otimes} E_{i}^{\varphi} \in \mu_{M}\left(F_{q, \varphi}^{M}[G]\right)$, hence $\left(F_{i}^{\varphi}\right)^{(f)} L_{-f \mu_{i}}^{\varphi, \otimes}, L_{-e \mu_{i}}^{\varphi, \otimes}\left(E_{i}^{\varphi}\right)^{(e)} \in$ $\mu_{M}\left(F_{q, \varphi}^{M}[G]\right)$ too; then Proposition 5.12(b) gives $\left(F_{i}^{\varphi}\right)^{(f)} L_{-f \mu_{i}}^{\varphi, \otimes}$, $L_{-e \mu_{i}}^{\varphi, \otimes}\left(E_{i}^{\varphi}\right)^{(e)} \in \mu_{M}\left(\mathcal{F}_{\varphi}^{M}[G]\right)$; similarly we find that $L_{-\mu_{i}}^{\varphi, \otimes}=\mu_{M}\left(z_{i}\right) \in$ $\mu_{M}\left(\mathcal{F}_{\varphi}^{M}[G]\right)$, with $z_{i}:=\psi_{-\mu_{i}} \in \mathcal{F}_{\varphi}^{M}[G]$. Then

$$
L_{\mu_{i}}^{\varphi, \otimes}=\left(\prod_{j=1}^{n} \widehat{i}_{-\mu_{j}}^{\varphi, \otimes}\right) \cdot L_{\rho}^{\varphi, \otimes} \in \mu_{M}\left(\mathcal{F}_{\varphi}^{M}[G]\left[\psi_{-\rho}^{-1}\right]\right)
$$

hence $\left(F_{i}^{\varphi}\right)^{(f)} \otimes 1,1 \otimes\left(E_{i}^{\varphi}\right)^{(e)} \in \mu_{M}\left(\mathcal{F}_{\varphi}^{M}[G]\left[\psi_{-\rho}^{-1}\right]\right) ;$ moreover, $\left(\begin{array}{c}L_{-\mu_{i} ; c}^{\varphi, \otimes} \\ t\end{array}\right)=\left(\begin{array}{c}\mu_{M}\left(z_{i}\right) ; c \\ t\end{array}\right)=\mu_{M}\left(\left(\begin{array}{c}z_{i} ; c \\ t\end{array}\right)\right)$, and $\left(\begin{array}{c}z_{i} ; c \\ t\end{array}\right) \in \mathcal{F}_{\varphi}^{M}[G]$, thus $\left(\begin{array}{c}L_{-\mu_{i}}^{\varphi, \otimes} ; c \\ t\end{array}\right) \in$ $\mu_{M}\left(\mathcal{F}_{\varphi}^{M}[G]\left[\psi_{-\rho}^{-1}\right]\right)$. Then Lemma 5.11 gives $\mu_{M}\left(\mathcal{F}_{\varphi}^{M}[G]\left[\psi_{-\rho}^{-1}\right]\right)=\mathcal{A}_{\varphi}^{M}$. The same can be done for the other integer form.

Now let $v_{\tau}$ be the image of $u_{\tau}$ (cf. the proof of Lemma 5.4) in the $k(q)$ algebra isomorphism $\theta: U_{0}^{M^{\prime}} \stackrel{\cong}{\cong} U_{0}^{M^{\prime}}$ given by $L_{\nu} \mapsto L_{-\nu}\left(\nu \in M^{\prime}\right)$ : Then $\left\{v_{\tau} \mid \tau \in M_{+}^{\prime} \cong \mathbb{N}^{n}\right\}$ is a basis of $U_{0}^{M^{\prime}}$; a quick review of the proof of Lemma 5.4 shows that $v_{\tau}^{*}$ (with respect to $\overline{i m}_{M}: U_{\varphi, 0}^{M} \hookrightarrow U_{0}^{M^{\prime *}}$ ) is a linear combination of elements $L_{-\mu}\left(\mu \in M_{+}\right)$. Then $j_{M}\left(L_{-\mu}\right)=L_{-\mu}^{\varphi, \otimes}$ (cf. (5.2)) and $L_{-\mu}^{\varphi, \otimes} \in \mu_{M}\left(F_{q, \varphi}^{M}[G]\right)$ imply $j_{M}\left(v_{\tau}^{*}\right) \in \mu_{M}\left(F_{q, \varphi}^{M}[G]\right)$, for all $\tau \in M_{+}$; since $L_{\mu} \in U_{0}^{M^{\prime *}}$ is a series of $v_{\tau}^{*}\left(\tau \in M_{+}^{\prime}\right)$ with coefficients in $k\left[q, q^{-1}\right]$, then $L_{\mu}^{\varphi, \otimes}=j_{M}\left(L_{\mu}\right)$ lies in the (topological) closure of $\mu_{M}\left(F_{q, \varphi}^{M}[G]\right)$, for all $\mu \in M_{+}$, so the same is true for $L_{\rho}^{\varphi, \otimes}=\mu_{M}\left(\psi_{-\rho}^{-1}\right)$ : This proves the denseness claim for $F_{q, \varphi}^{M}[G]$. As $L_{-\mu}^{\varphi, \otimes} \in \mu_{M}\left(\mathfrak{F}_{\varphi}^{M}[G]\right), L_{-\mu}^{\varphi, \otimes} \in \mu_{M}\left(\mathcal{F}_{\varphi}^{M}[G]\right)$, this argument works for integer forms too.

5.15. Gradings. Recall that $U_{\varphi, \geq}^{M}$ has a $Q_{+}$-grading $U_{\varphi, \geq}^{M}=\oplus_{\alpha \in Q_{+}}\left(U_{\geq}^{M}\right)_{\alpha}$ given by decomposition in direct sum of weight spaces for the adjoint action of $U_{\varphi, 0}^{M}$; also $U_{\varphi, \leq}^{M}$ has an analogous $Q_{-}$-grading. These are gradings of Hopf algebras (in the usual obvious sense), inherited by the integer forms, and 
DRT pairings respect them, that is e.g., $\pi\left(\left(U_{\varphi, \leq}^{M}\right)_{\beta},\left(U_{\varphi, \geq}^{M^{\prime}}\right)_{\gamma}\right)=0$ for all $\beta \in Q_{-}, \gamma \in Q_{+}$such that $\beta+\gamma \neq 0$.

The gradings of quantum Borel subalgebras induce a $Q$-grading of the Hopf algebra $D_{M}:=U_{\varphi, \geq}^{M} \otimes U_{\varphi, \leq}^{Q}$ (inherited by its quotient Hopf algebra $\left.U_{q, \varphi}^{M}(\mathfrak{g})\right)$, where the subspace $\left(U_{\varphi, \geq}^{M}\right)_{\beta} \otimes\left(U_{\varphi, \leq}^{Q}\right)_{\gamma}$ has degree $\beta+\gamma$, and also a $Q$-grading of the subalgebra $U_{\varphi, \geq}^{M} \otimes U_{\varphi, \leq}^{Q}$ of $D_{M^{\prime}}{ }^{*}$; since $D_{M^{\prime}}{ }^{*}$ is a completion (via formal series) of this subalgebra, it inherits on its own sort of a "pseudograding", in the sense that every element of $D_{M^{\prime}}{ }^{*}$ is a (possibly infinite) sum of terms each of whom has a well-defined degree: Namely, given $f \in D_{M^{\prime}}{ }^{*}$ with formal series expansion (cf. Remark 5.5) $f=\sum_{\mathcal{F} \varphi, \phi, \mathcal{E}^{\varphi}} \mathcal{F}^{\varphi} \cdot \phi \cdot \mathcal{E}^{\varphi}$ (where $\phi \in\left(U_{\varphi, 0}^{M^{\prime}} \otimes U_{\varphi, 0}^{Q}\right)^{*}$, and $\mathcal{F}^{\varphi}$ 's and $\mathcal{E}^{\varphi}$ 's are PBW monomials), we define the degrees of its various summands as given by

$$
\operatorname{deg}\left(\mathcal{F}^{\varphi} \cdot \phi \cdot \mathcal{E}^{\varphi}\right):=\operatorname{deg}\left(\mathcal{F}^{\varphi}\right)+\operatorname{deg}\left(\mathcal{E}^{\varphi}\right)
$$

where $\operatorname{deg}\left(\prod_{r=N}^{1}\left(F_{\alpha^{r}}^{\varphi}\right)^{f_{r}}\right):=-\sum_{r=1}^{N} f_{r} \alpha^{r}, \quad \operatorname{deg}\left(\prod_{r=1}^{N}\left(E_{\alpha^{r}}^{\varphi}\right)^{e_{r}}\right):=$ $\sum_{r=1}^{N} e_{r} \alpha^{r}$ (this degree is again a weight for a suitable action of $U_{\varphi, 0}^{M}$ on $\left.U_{\varphi, \leq}^{M} \otimes U_{\varphi, \geq}^{P}\right)$. Now $U_{\varphi, \leq}^{M} \otimes U_{\varphi, \geq}^{P}$ is dense in $D_{M^{\prime}}{ }^{*}$, and the restriction of the pairing $D_{M^{\prime}}{ }^{*} \otimes D_{M^{\prime}} \rightarrow k(q)$ to $\left(U_{\varphi, \leq}^{M} \otimes U_{\varphi, \geq}^{P}\right) \otimes\left(U_{\varphi, \geq}^{M^{\prime}} \otimes U_{\varphi, \leq}^{Q}\right)$ is nothing but $\left(\pi_{\varphi} \otimes \bar{\pi}_{\varphi}\right) \circ \tau_{2,3}$ (with $\tau_{2,3}: x \otimes y \otimes z \otimes w \mapsto x \otimes z \otimes y \otimes w ;$ ) therefore, since $\pi_{\varphi}$ and $\bar{\pi}_{\varphi}$ respect the gradings, also the pairing $D_{M^{\prime}}{ }^{*} \otimes D_{M^{\prime}} \rightarrow k(q)$ respects the pseudogradings we are dealing with.

Finally, the pseudograding of $D_{M^{\prime}}{ }^{*}$ is compatible with the formal Hopf structure. For example, look at $S(x)$, for homogeneous $x \in D_{M^{\prime}}{ }^{*}$ : given homogeneous $y \in D_{M^{\prime}}$, we have $\langle S(x), y\rangle=\langle x, S(y)\rangle=\left\langle x, y^{\prime}\right\rangle$ where $y^{\prime}:=S(y)$ is homogeneous on its own of degree $\operatorname{deg}\left(y^{\prime}\right)=\operatorname{deg}(y)$ (for the grading of $D_{M^{\prime}}$ is compatible with the Hopf structure); therefore

$$
\langle S(x), y\rangle \neq 0 \Longrightarrow \operatorname{deg}(y)=\operatorname{deg}\left(y^{\prime}\right)=\operatorname{deg}(x) \Longrightarrow S(x) \in\left(D_{M^{\prime}}{ }^{*}\right) \operatorname{deg}(x)
$$

that is $\operatorname{deg}(S(x))=\operatorname{deg}(x)$, q.e.d.

5.16. Umbral calculus. In this section we provide concrete information about the Hopf structure of our quantum formal groups. This will be especially important for defining integer forms and specializing them at roots of 1.

The counit $\epsilon: D_{M^{\prime}}{ }^{*} \rightarrow k(q)$ is $\epsilon:=1^{*}$, hence $\epsilon\left(x^{*}\right):=\left\langle x^{*}, 1\right\rangle$ for all $x^{*} \in D_{M^{\prime}}{ }^{*} ;$ thus

$$
\epsilon\left(F_{i}^{\varphi} \otimes 1\right)=0, \quad \epsilon\left(L_{\mu}^{\varphi, \otimes}\right)=1, \quad \epsilon\left(1 \otimes E_{i}\right)=0 ;
$$

the elements above generate the algebra $j_{M}\left(U_{q}^{M^{\prime}}(\mathfrak{g})^{*}\right.$ ) (in topological sense, cf. Theorem 5.14), hence (5.4) uniquely determines $\epsilon: j_{M}\left(U_{q, \varphi}^{M^{\prime}}(\mathfrak{g})^{*}\right) \longrightarrow$ $k(q)$. 
The antipode of $D_{M^{\prime}}{ }^{*}$ is by definition the dual of the antipode of $D_{M^{\prime}}$, hence it is characterized by $\left\langle S\left(x^{*}\right), x\right\rangle=\left\langle x^{*}, S(x)\right\rangle$, for all $x^{*} \in D_{M^{\prime}}{ }^{*}$, $x \in D_{M^{\prime}}$. Now consider $\left(F_{i}^{\varphi}\right)^{f} \otimes 1 \in U_{\varphi, \leq}^{M} \otimes U_{\varphi, \geq}^{P} \leq D_{M^{\prime}}{ }^{*}, f \in \mathbb{N}$ : it is homogeneous of degree $-f \alpha_{i}$, whence $S\left(\left(F_{i}^{\varphi}\right)^{f} \otimes 1\right)$ has the same degree. Thus writing $S\left(\left(F_{i}^{\varphi}\right)^{f} \otimes 1\right)$ as a series

$$
S\left(\left(F_{i}^{\varphi}\right)^{f} \otimes 1\right)=\sum_{\sigma} F_{\sigma} \cdot \Phi_{\sigma} \cdot E_{\sigma}
$$

we have $\operatorname{deg}\left(F_{\sigma}^{\varphi} \cdot \Phi_{\sigma} \cdot E_{\sigma}^{\varphi}\right):=\operatorname{deg}\left(F_{\sigma}^{\varphi}\right)+\operatorname{deg}\left(E_{\sigma}^{\varphi}\right)=-f \alpha_{i}$. Now, the pseudograding of $D_{M^{\prime}}{ }^{*}$ induces a pseudograding of $\mathfrak{I}_{\varphi}^{M}$ too; hence, since $\mathfrak{I}_{\varphi}^{M}$ is a formal Hopf subalgebra of $D_{M^{\prime}}{ }^{*}$ (Proposition 5.9), we can apply the same procedure and get

$$
S\left(\left(\bar{F}_{i}^{\varphi}\right)^{f} \otimes 1\right)=\sum_{\sigma} \mathcal{F}_{\sigma}^{\varphi} \cdot \varphi_{\sigma} \cdot \mathcal{E}_{\sigma}^{\varphi}
$$

where $\varphi_{\sigma} \in \mathfrak{U}_{\varphi, 0}^{M^{\prime *}}$ and the $\mathcal{F}_{\sigma}^{\varphi}$ 's, resp. $\mathcal{E}_{\sigma}^{\varphi}$ 's, are PBW monomials of $\mathcal{U}_{\varphi,-}$, resp. $\mathcal{U}_{\varphi,+}$, such that $\operatorname{deg}\left(\mathcal{F}_{\sigma}^{\varphi}\right)+\operatorname{deg}\left(\mathcal{E}_{\sigma}^{\varphi}\right)=-f \alpha_{i}$. An entirely similar argument yields

$$
S\left(\left(F_{i}^{\varphi}\right)^{(f)} \otimes 1\right)=\sum_{\sigma} \mathfrak{F}_{\sigma}^{\varphi} \cdot \phi_{\sigma} \cdot \mathfrak{E}_{\sigma}^{\varphi}
$$

where $\phi_{\sigma} \in \mathcal{U}_{0}^{M^{\prime *}}$ and the $\mathfrak{F}_{\sigma}^{\varphi}$, s, resp. $\mathfrak{E}_{\sigma}^{\varphi}$, are PBW monomials of $\mathfrak{U}_{\varphi,-}$, resp. $\mathfrak{U}_{\varphi,+}$, such that $\operatorname{deg}\left(\mathfrak{F}_{\sigma}^{\varphi}\right)+\operatorname{deg}\left(\mathfrak{E}_{\sigma}^{\varphi}\right)=-f \alpha_{i}$. Now remark that $\mathfrak{I}_{\varphi}^{M}$ and $\mathcal{I}_{\varphi}^{M}$ can be compared through the natural embedding $\mathcal{I}_{\varphi}^{M} \cong \mathfrak{U}_{\varphi}^{M^{\prime}}(\mathfrak{g})^{*} \hookrightarrow$ $\mathcal{U}_{\varphi}^{M^{\prime}}(\mathfrak{g})^{*} \cong \mathfrak{I}_{\varphi}^{M}\left(\right.$ dual of $\left.\mathcal{U}_{\varphi}^{M^{\prime}}(\mathfrak{g}) \hookrightarrow \mathfrak{U}_{\varphi}^{M^{\prime}}(\mathfrak{g})\right) ;$ since $\left(\bar{F}_{\alpha^{h}}^{\varphi}\right)^{f}=\prod_{s=1}^{f}\left(q_{\alpha^{h}}^{s}-q_{\alpha^{h}}^{-s}\right)$. $\left(F_{\alpha^{h}}^{\varphi}\right)^{(f)},\left(\bar{E}_{\alpha^{k}}^{\varphi}\right)^{e}=\prod_{s=1}^{f}\left(q_{\alpha^{k}}^{s}-q_{\alpha^{k}}^{-s}\right) \cdot\left(E_{\alpha^{k}}^{\varphi}\right)^{(e)}$, comparing (5.5) and (5.6) we find

$$
\mathfrak{F}_{\sigma}^{\varphi} \cdot \phi_{\sigma} \cdot \mathfrak{E}_{\sigma}^{\varphi} \in \prod_{h, k=1}^{n} \prod_{r=1}^{f_{h}} \prod_{s=1}^{e_{k}}\left(q_{\alpha^{h}}^{r}-q_{\alpha^{h}}^{-r}\right) \cdot\left(q_{\alpha^{k}}^{s}-q_{\alpha^{k}}^{-s}\right) \cdot \prod_{u=1}^{f}\left(q_{i}^{u}-q_{i}^{-u}\right)^{-1} \cdot \mathcal{I}_{\varphi}^{M}
$$

for $\mathfrak{F}_{\sigma}^{\varphi}=\prod_{h=N}^{1}\left(F_{\alpha^{h}}^{\varphi}\right)^{\left(f_{h}\right)}, \mathfrak{E}_{\sigma}^{\varphi}=\prod_{k=1}^{N}\left(E_{\alpha^{k}}^{\varphi}\right)^{\left(e_{k}\right)}$. Therefore

$$
\begin{aligned}
& S\left(\left(F_{i}^{\varphi}\right)^{(f)} \otimes 1\right)= \\
& =\sum_{\sigma} \prod_{h, k=1}^{n} \frac{\prod_{r=1}^{f_{h}} \prod_{s=1}^{e_{k}}\left(q_{\alpha^{h}}^{r}-q_{\alpha^{h}}^{-r}\right) \cdot\left(q_{\alpha^{k}}^{s}-q_{\alpha^{k}}^{-s}\right)}{\prod_{u=1}^{f}\left(q_{i}^{u}-q_{i}^{-u}\right)} \cdot \mathfrak{F}_{\sigma}^{\varphi} \cdot \phi_{\sigma}^{\prime} \cdot \mathfrak{E}_{\sigma}^{\varphi}
\end{aligned}
$$

in particular from every coefficient in (5.7) we can pick out a factor of type $\prod_{h=1}^{N} \prod_{r=1}^{a_{h}} \prod_{s=1}^{b_{h}}\left(q^{r}-q^{-r}\right) \cdot\left(q^{s}-q^{-s}\right)$ with $\sum_{h=1}^{N}\left(a_{h}+b_{h}\right)=\sum_{h=1}^{N}\left(f_{h}+\right.$ 
$\left.e_{k}\right)-f$; then we can rearrange the terms of the series (5.7) and write it again as

$$
\begin{aligned}
& S\left(\left(F_{i}^{\varphi}\right)^{(f)} \otimes 1\right)= \\
& =\sum_{n=0}^{+\infty} \sum_{\sum_{h} a_{h}+b_{h}=n} \prod_{h=1}^{N} \prod_{r=1}^{a_{h}} \prod_{s=1}^{b_{h}}\left(q^{r}-q^{-r}\right) \cdot\left(q^{s}-q^{-s}\right) \cdot X_{n}
\end{aligned}
$$

where $X_{n} \in \mathfrak{U}_{\varphi,-} \otimes \mathcal{U}_{\varphi, 0}^{M^{\prime *}} \otimes \mathfrak{U}_{\varphi,+}$. Similarly occurs for the other generators of $\mathcal{A}_{\varphi}^{M}$ : Thus

For any root of unity $\varepsilon$, the series $S\left(\left(F_{i}^{\varphi}\right)^{(f)} \otimes 1\right), S\left(\left(\begin{array}{c}L_{\mu_{i}}^{\varphi, \otimes} ; c \\ t\end{array}\right)\right)$, $S\left(L_{-\mu_{i}}^{\varphi, \otimes}\right)$ and $S\left(1 \otimes\left(E_{i}^{\varphi}\right)^{(e)}\right)$ are finite sums modulo $(q-\varepsilon)$.

In principle, one can compute all the terms of these series up to any fixed order $n$; actually, we need to know them only up to $n=0$. For $S\left(F_{i}^{\varphi} \otimes 1\right)$ the first term (call it $\mathbf{F}_{1}$ ), with index $n=0$ in (5.8), corresponds to the terms $\mathfrak{F}_{\sigma}^{\varphi} \cdot \phi_{\sigma} \cdot \mathfrak{E}_{\sigma}$ in (5.6) such that $\sum_{s=1}^{N}\left(f_{s}+e_{s}\right)=1$; but these must have degree $\operatorname{deg}\left(\mathfrak{F}_{\sigma}^{\varphi}\right)+\operatorname{deg}\left(\mathfrak{E}_{\sigma}^{\varphi}\right)=-\alpha_{i}$ too, whence $\mathfrak{F}_{\sigma}^{\varphi}=F_{i}$ and $\mathfrak{E}_{\sigma}^{\varphi}=1$. Now, $\mathbf{F}_{1}$ takes non-zero values only on the free $\mathcal{U}_{0}^{M^{\prime}}$-module with basis $\left\{\bar{E}_{i}\right\}$, call it $V_{1, i}$ : Direct computation shows that $\mathbf{F}_{1}+q_{i}^{\left(\alpha_{i} \mid \alpha_{i}+\tau_{i}\right)} \cdot F_{i}^{\varphi} L_{-\alpha_{i}}^{\varphi, \otimes}$ is zero in $V_{1, i}^{*}$, therefore $\mathbf{F}_{1}=-q_{i}^{\left(\alpha_{i} \mid \alpha_{i}+\tau_{i}\right)} \cdot F_{i}^{\varphi} L_{-\alpha_{i}}^{\varphi, \otimes}$, whence

$$
S\left(F_{i}^{\varphi} \otimes 1\right) \equiv-q^{-\left(\alpha_{i} \mid \alpha_{i}+\tau_{i}\right)} \cdot F_{i}^{\varphi} L_{-\alpha_{i}}^{\varphi, \otimes} \quad \bmod \left(q-q^{-1}\right) .
$$

Similar arguments give

$$
\begin{aligned}
S\left(\left(\begin{array}{c}
L_{\mu_{i}}^{\varphi, \otimes} ; 0 \\
1
\end{array}\right)\right) & \equiv-L_{-\mu_{i}}^{\varphi, \otimes} \cdot\left(\begin{array}{c}
L_{\mu_{i}}^{\varphi, \otimes} ; 0 \\
1
\end{array}\right) \quad \bmod \left(q-q^{-1}\right) \\
S\left(1 \otimes E_{i}^{\varphi}\right) & \equiv-q^{+\left(\alpha_{i} \mid \alpha_{i}-\tau_{i}\right)} \cdot L_{-\alpha_{i}}^{\varphi, \otimes} E_{i}^{\varphi}
\end{aligned}
$$

As for the coproduct $\Delta: D_{M^{\prime}}{ }^{*} \rightarrow D_{M^{\prime}}{ }^{*} \widehat{\otimes} D_{M^{\prime}}{ }^{*}$, it is the dual of the product of $D_{M^{\prime}}$, hence it is characterized by $\left\langle\Delta\left(x^{*}\right), y \otimes z\right\rangle=\left\langle x^{*}, y \cdot z\right\rangle$. Mimicking the procedure used for $S$, we find that $\Delta\left(\left(F_{i}^{\varphi}\right)^{(f)} \otimes 1\right)$ is given by a series of type

$$
\begin{aligned}
\Delta\left(\left(F_{i}^{\varphi}\right)^{(f)} \otimes 1\right)=\sum_{n=0}^{+\infty} \sum_{\substack{\sum_{h}\left(a_{h}+a_{h}^{\prime}+\\
+b_{h}+b_{h}^{\prime}\right)=n \\
a_{h}^{\prime}}} \prod_{h=1}^{N} \prod_{r=1}^{a_{h}} \prod_{s=1}^{b_{h}}\left(q^{r}-q^{-r}\right) \cdot\left(q^{s}-q^{-s}\right) \\
\cdot \prod_{r^{\prime}=1}^{b_{h}^{\prime}} \prod_{s^{\prime}=1}\left(q^{r^{\prime}}-q^{-r^{\prime}}\right) \cdot\left(q^{s^{\prime}}-q^{-s^{\prime}}\right) \cdot Y_{n}
\end{aligned}
$$


in which $Y_{n} \in\left(\mathfrak{U}_{\varphi,-} \otimes \mathcal{U}_{\varphi, 0}^{M^{\prime *}} \otimes \mathfrak{U}_{\varphi,+}\right)^{\otimes 2}$. Similar formulas exist for all the generators of $\mathcal{A}_{\varphi}^{M}\left(\right.$ which are topological generators of $\left.j_{M}\left(U_{q, \varphi}^{M^{\prime}}(\mathfrak{g})^{*}\right)\right)$ : In particular this implies:

For any root of unity $\varepsilon$, the series $\Delta\left(\left(F_{i}^{\varphi}\right)^{(f)} \otimes 1\right), \Delta\left(\left(\begin{array}{c}L_{\mu_{i}}^{\varphi, \otimes} ; c \\ t \\ { }_{t}\end{array}\right)\right)$, $\Delta\left(L_{-\mu_{i}}^{\varphi, \otimes}\right)$ and $\Delta\left(1 \otimes\left(E_{i}^{\varphi}\right)^{(e)}\right)$ are finite sums modulo $(q-\varepsilon)$.

Direct computation gives us the following congruences modulo $\left(q-q^{-1}\right)^{2}$ (using notation $F_{i}^{\varphi, \otimes},:=F_{i}^{\varphi} \otimes 1, L_{\mu}^{\varphi, \otimes}:=L_{-(1+\varphi) \mu} \otimes L_{(1-\varphi)(\mu)}, E_{i}^{\varphi, \otimes}:=$ $1 \otimes E_{i}^{\varphi}$, and so on)

$$
\begin{aligned}
\Delta & \left(F_{i}^{\varphi, \otimes}\right) \equiv \\
\equiv & F_{i}^{\varphi, \otimes} \otimes 1^{\otimes}+1^{\otimes} \otimes F_{i}^{\varphi, \otimes}+\left(q_{i}-1\right) \cdot\left(\begin{array}{c}
L_{\alpha_{i}}^{\varphi, \otimes} ; 0 \\
1
\end{array}\right) \otimes F_{i}^{\varphi, \otimes} \\
& +\left(q_{i}-q_{i}^{-1}\right)^{-1} \cdot \sum_{\alpha, \beta \in R^{+}} C_{\alpha, \beta}^{i,+}\left(q_{\alpha}-q_{\alpha}^{-1}\right)\left(q_{\beta}-q_{\beta}^{-1}\right) \cdot L_{\alpha_{i}}^{\varphi, \otimes} E_{\alpha}^{\varphi, \otimes} \otimes F_{\beta}^{\varphi, \otimes} \\
& \left(\left(\begin{array}{c}
L_{\mu_{i}}^{\varphi, \otimes} ; 0 \\
1
\end{array}\right)\right) \equiv \\
\equiv & \left(\begin{array}{c}
L_{\mu_{i}}^{\varphi, \otimes} ; 0 \\
1
\end{array}\right) \otimes 1^{\otimes}+1^{\otimes} \otimes\left(\begin{array}{c}
L_{\mu_{i}}^{\varphi, \otimes} ; 0 \\
1
\end{array}\right) \\
& +\left(q_{i}-1\right) \cdot\left(\begin{array}{c}
L_{\mu_{i}}^{\varphi, \otimes} ; 0 \\
1
\end{array}\right) \otimes\left(\begin{array}{c}
L_{\mu_{i}}^{\varphi, \otimes} ; 0 \\
1
\end{array}\right) \\
& +(2)_{q^{-1}}^{2}\left(d_{i}\right)_{q}^{-1} \cdot \sum_{\gamma \in R^{+}}(q-1)\left[d_{\gamma}\right]_{q}\left[\left(\mu_{i} \mid \gamma\right)\right]_{q} \cdot L_{\mu_{i}}^{\varphi, \otimes} E_{\gamma}^{\varphi, \otimes} \otimes F_{\gamma}^{\varphi, \otimes} L_{\mu_{i}}^{\varphi, \otimes} \\
\Delta & \left(E_{i}^{\varphi, \otimes}\right) \equiv{ }^{\otimes} \otimes E_{i}^{\varphi, \otimes}+E_{i}^{\varphi, \otimes} \otimes 1^{\otimes}+\left(q_{i}-1\right) \cdot E_{i}^{\varphi, \otimes} \otimes\left(\begin{array}{c}
L_{\alpha_{i}}^{\varphi, \otimes} ; 0 \\
1
\end{array}\right) \\
\equiv & -\left(q_{i}-q_{i}^{-1}\right)^{-1} \cdot \sum_{\alpha, \beta \in R^{+}} C_{\alpha, \beta}^{i,-}\left(q_{\alpha}-q_{\alpha}^{-1}\right)\left(q_{\beta}-q_{\beta}^{-1}\right) E_{\alpha}^{\varphi, \otimes} \otimes F_{\beta}^{\varphi, \otimes} L_{\alpha_{i}}^{\varphi, \otimes}
\end{aligned}
$$

where the $C_{\alpha, \beta}^{i, \pm}$, s are given by the equations $\pi_{i}^{-}\left(\left[F_{\alpha}, E_{\beta}\right]\right)=C_{\alpha, \beta}^{i,-} \cdot F_{i}$, $\pi_{i}^{+}\left(\left[F_{\alpha}, E_{\beta}\right]\right)=C_{\alpha, \beta}^{i,+} \cdot E_{i}\left(\pi_{i}^{-}: U_{q}^{Q}(\mathfrak{g}) \rightarrow k(q) \cdot F_{i}\right.$ and $\pi_{i}^{+}: U_{q}^{Q}(\mathfrak{g}) \rightarrow k(q) \cdot E_{i}$ being the canonical maps).

\section{The quantum group $U_{q, \varphi}^{M}(\mathfrak{h})$.}


6.1. The quantum enveloping algebra $U_{q, \varphi}^{M}(\mathfrak{h})$. The results of $\S 5$ can be given an axiomatic form: To this end, we introduce a new object $U_{q, \varphi}^{M}(\mathfrak{h})$ which is with respect to $U\left(\mathfrak{h}^{\tau}\right)$ what $U_{q, \varphi}^{M}(\mathfrak{g})$ is for $U\left(\mathfrak{g}^{\tau}\right)$. Here $M$ is a fixed lattice as in $\S 2.2$.

We define $\mathbf{H}_{\varphi}^{M}$ to be the associative $k(q)$-algebra with 1 with generators

$$
F_{i}^{\varphi}, L_{\mu}^{\varphi}, E_{i}^{\varphi} \quad(\lambda \in M ; i=1, \ldots, n)
$$

and relations

$$
\begin{aligned}
& L_{0}^{\varphi}=1, \quad L_{\mu}^{\varphi} L_{\nu}^{\varphi}=L_{\mu+\nu}^{\varphi}, \quad E_{i}^{\varphi} F_{j}^{\varphi}-F_{j}^{\varphi} E_{i}^{\varphi}=0 \\
& L_{\mu}^{\varphi} F_{j}^{\varphi}=q^{\left(\alpha_{j} \mid(1+\varphi)(\mu)\right)} F_{j}^{\varphi} L_{\mu}^{\varphi}, \quad L_{\mu}^{\varphi} E_{j}^{\varphi}=q^{\left(\alpha_{j} \mid(1-\varphi)(\mu)\right)} E_{j}^{\varphi} L_{\mu}^{\varphi} \\
& \sum_{k=0}^{1-a_{i j}}(-1)^{k} q^{+c_{i j}^{k}}\left[\begin{array}{c}
1-a_{i j} \\
k
\end{array}\right]_{q_{i}}\left(E_{i}^{\varphi}\right)^{1-a_{i j}-k} E_{j}^{\varphi}\left(E_{i}^{\varphi}\right)^{k}=0 \quad \forall i \neq j \\
& \sum_{k=0}^{1-a_{i j}}(-1)^{k} q^{-c_{i j}^{k}}\left[\begin{array}{c}
1-a_{i j} \\
k
\end{array}\right]_{q_{i}}\left(F_{i}^{\varphi}\right)^{1-a_{i j}-k} F_{j}^{\varphi}\left(F_{i}^{\varphi}\right)^{k}=0 \quad \forall i \neq j
\end{aligned}
$$

where $c_{i j}^{k}:=-\left(k \alpha_{i} \mid \tau_{j}+\left(1-a_{i j}-k\right) \tau_{i}\right)-\left(\alpha_{j} \mid\left(1-a_{i j}-k\right) \tau_{i}\right)$ for all $i$, $j, k$. We also use notation $M_{i}^{\varphi}:=L_{\mu_{i}}^{\varphi}(i=1, \ldots, n),\left\{\mu_{1}, \ldots, \mu_{n}\right\}$ being a fixed $\mathbb{Z}$-basis of $M$, cf. $\S 1.1$.

Now consider $F_{\alpha^{1}}^{\varphi}, \ldots, F_{\alpha^{N}}^{\varphi}$ in $U_{\varphi,-}\left(\subseteq \mathbf{H}_{\varphi}^{M}\right)$, the elements $B_{\eta, \tau, \phi}^{\varphi}:=$ $\sum_{\tau^{\prime} \preceq \tau} c_{\tau, \tau^{\prime}}^{\prime} \cdot L_{\tau^{\prime}}^{\varphi}$ (cf. §5.6) in $U_{\varphi, 0}^{M}\left(\subseteq \mathbf{H}_{\varphi}^{M}\right)$, and $E_{\alpha^{1}}^{\varphi}, \ldots, E_{\alpha^{N}}^{\varphi}$ in $U_{\varphi,+}(\subseteq$ $\left.\mathbf{H}_{\varphi}^{M}\right)$.

We define $U_{q, \varphi}^{M}(\mathfrak{h})$ to be the completion of $\mathbf{H}_{M}$ by means of formal series, with coefficients in $k(q)$, in the elements of the set

$$
\begin{aligned}
& \mathbb{B}_{M}^{\varphi}:= \\
& :=\left\{\prod_{r=N}^{1}\left(F_{\alpha^{r}}^{\varphi}\right)^{f_{r}} \cdot B_{\eta, \tau, \phi}^{\varphi} \cdot \prod_{r=1}^{N}\left(E_{\alpha^{r}}^{\varphi}\right)^{e_{r}} \mid \phi=\left(f_{r}\right)_{r}, \eta=\left(e_{r}\right)_{r} \in \mathbb{N}^{N} ; \tau \in \mathbb{N}^{n}\right\} .
\end{aligned}
$$

Thus $U_{q, \varphi}^{M}(\mathfrak{h})$ is the completion of $\mathbf{H}_{\varphi}^{M}$ with respect to the topology (of $\mathbf{H}_{\varphi}^{M}$ ) for which a fundamental system of neighborhoods of 0 is the set of vector subspaces of $\mathbf{H}_{\varphi}^{M}$ which contain almost all the elements of $\mathbb{B}_{M}^{\varphi}$, and the set $\mathbb{B}_{M}^{\varphi}$ is a pseudobasis of $U_{q, \varphi}^{M}(\mathfrak{h})$. Roughly speaking, $U_{q, \varphi}^{M}(\mathfrak{h})$ is an algebra of (non-commutative) formal series with (6.1) as commutation rules. Finally, thanks to Lemma 5.3, we can identify $U_{q, \varphi}^{M}(\mathfrak{h})$ with the space of formal series in the $F_{\alpha^{h}}^{\varphi}$ 's, $E_{\alpha^{k}}^{\varphi}$ 's with coefficients in $U_{\varphi, 0}^{M^{\prime *}}$.

From $\S 5$ we can explicitely realize $U_{q, \varphi}^{M}(\mathfrak{h})$ and endow it with a Hopf structure: In fact, the definition of $U_{q, \varphi}^{M}(\mathfrak{h})$ is nothing but a presentation of $U_{q, \varphi}^{M^{\prime}}(\mathfrak{g})^{*}$, as the following shows: 
Theorem 6.2. $\quad$ There exists an isomorphism of topological $k(q)$-algebras

$$
\nu_{M}^{\varphi}: U_{q, \varphi}^{M}(\mathfrak{h}) \stackrel{\cong}{\longrightarrow} j_{M}\left(U_{q, \varphi}^{M^{\prime}}(\mathfrak{g})^{*}\right)
$$

given by: $F_{i}^{\varphi} \mapsto F_{i}^{\varphi} \otimes 1, L_{\mu}^{\varphi} \mapsto L_{\mu}^{\varphi, \otimes}, E_{i}^{\varphi} \mapsto 1 \otimes E_{i}^{\varphi}$. Then the pull-back of the formal Hopf structure of $j_{M}\left(U_{q, \varphi}^{M^{\prime}}(\mathfrak{g})^{*}\right)$ uniquely defines a formal Hopf structure on $U_{q, \varphi}^{M}(\mathfrak{h})$, so that $\nu_{M}^{\varphi}$ and $j_{M}{ }^{-1} \circ \nu_{M}^{\varphi}$ are formal Hopf algebra isomorphisms.

Proof. By construction $\mathbf{H}_{\varphi}^{M} \cong U_{\varphi,-} \otimes U_{\varphi, 0}^{M} \otimes U_{\varphi,+} \cong A_{\varphi}^{M}\left(\subseteq j_{M}\left(U_{q, \varphi}^{M^{\prime}}(\mathfrak{g})^{*}\right)\right)$ as vector spaces; now $F_{i}^{\varphi} \otimes 1, L_{\mu}^{\varphi, \otimes}, 1 \otimes E_{i}^{\varphi} \in U_{\varphi, \leq}^{M} \otimes U_{\varphi, \geq}^{P}$, hence comparing (6.1) and (2.1) we see that formulas above gives a well-defined isomorphism of algebras $\nu_{M}^{\varphi}: \mathbf{H}_{\varphi}^{M} \stackrel{\cong}{\longrightarrow} A_{\varphi}^{M}$. Moreover, $A_{\varphi}^{M}$ contains a pseudobasis $\mathbf{B}_{M}^{\varphi}$ of $j_{M}\left(U_{q, \varphi}^{M^{\prime}}(\mathfrak{g})^{*}\right)$ (cf. Lemma 5.4, Proposition 5.6, and Remark 5.7) such that $\nu_{M}^{\varphi}\left(\mathbb{B}_{M}^{\varphi}\right)=\mathbf{B}_{M}^{\varphi}$, hence $\nu_{M}^{\varphi}$ continuosly extends, in a unique way, to an isomorphism of topological algebras $\nu_{M}: U_{q, \varphi}^{M}(\mathfrak{h}) \stackrel{\cong}{\longrightarrow} j_{M}\left(U_{q, \varphi}^{M^{\prime}}(\mathfrak{g})^{*}\right)$, q.e.d.

Remark 6.3. Notice that. setting $Y_{\eta, \tau, \phi}^{\varphi}:=\mathcal{F}_{\eta}^{\varphi} \cdot B_{\eta, \tau, \phi}^{\varphi} \cdot \mathcal{E}_{\phi}^{\varphi}$, (notations of $\S 5)$, Theorem 6.2 and definitions give $\nu_{M}^{\varphi}\left(Y_{\eta, \tau, \phi}^{\varphi}\right)=X_{\eta, \tau, \phi}{ }^{*}$ for all $\eta \in \mathbb{N}^{N}$, $\tau \in \mathbb{N}^{n}, \phi \in \mathbb{N}^{N}$ (cf. $\left.\S 5.7\right)$.

Lemma 6.4. The subset $\Omega_{\varphi}^{M}:=\left\{x=\sum_{\sigma} F_{\sigma}^{\varphi} \cdot \Phi_{\sigma}^{\varphi} \cdot E_{\sigma}^{\varphi} \in U_{q, \varphi}^{M}(\mathfrak{h}) \mid \Phi_{\sigma}^{\varphi} \in\right.$ $\left.U_{\varphi, 0}^{M}, \forall \sigma\right\}$ (where $x=\sum_{\sigma} F_{\sigma}^{\varphi} \cdot \Phi_{\sigma}^{\varphi} \cdot E_{\sigma}^{\varphi}$ is the expansion of $x \in U_{q, \varphi}^{M}(\mathfrak{h})$ as a series with coefficients in $\left.U_{\varphi, 0}^{M^{\prime *}}\right)$ is a formal Hopf subalgebra of $U_{q, \varphi}^{M}(\mathfrak{h})$.

Proof. It is clear that $\Omega_{\varphi}^{M}$ is a subalgebra of $U_{q, \varphi}^{M}(\mathfrak{h})$. Now let $x=\sum_{\tau} F_{\tau}^{\varphi^{\prime}}$. $\Phi_{\tau}^{\varphi \prime} \cdot E_{\tau}^{\varphi \prime} \in \Omega_{\varphi}^{M}$ : then $\Phi_{\tau}^{\varphi \prime}=\sum_{\mu \in M} c_{\tau, \mu} L_{\mu}^{\varphi}$ with $c_{\tau, \mu} \neq 0$ for finitely many $\mu$.

Let $S(x)=\sum_{\sigma} F_{\sigma}^{\varphi} \cdot \Phi_{\sigma}^{\varphi} \cdot E_{\sigma}^{\varphi}$ : For any fixed $\bar{\sigma}$, we must prove that $\Phi_{\bar{\sigma}}^{\varphi} \in U_{\varphi, 0}^{M}\left(\subseteq U_{0}^{M^{\prime *}}\right)$, so that $S\left(\Omega_{\varphi}^{M}\right)=\Omega_{\varphi}^{M}$; to this end, we use the identification $U_{q, \varphi}^{M}(\mathfrak{h}) \cong U_{q, \varphi}^{M^{\prime}}(\mathfrak{g})^{*}$ (cf. Theorem 6.2). For (2.3) there exist two PBW monomials $\mathcal{E}_{\bar{\sigma}}$ and $\mathcal{F}_{\bar{\sigma}}$ such that

$\left\langle S(x), \mathcal{E}_{\bar{\sigma}} \cdot y \cdot \mathcal{F}_{\bar{\sigma}}\right\rangle=\left\langle F_{\bar{\sigma}}^{\varphi} \cdot \Phi_{\bar{\sigma}}^{\varphi} \cdot E_{\bar{\sigma}}^{\varphi}, \mathcal{E}_{\bar{\sigma}} \cdot y \cdot \mathcal{F}_{\bar{\sigma}}\right\rangle=\left\langle F_{\bar{\sigma}}, \mathcal{E}_{\bar{\sigma}}\right\rangle \cdot\left\langle E_{\bar{\sigma}}, \mathcal{F}_{\bar{\sigma}}\right\rangle \cdot \Phi_{\bar{\sigma}}^{\varphi}\left(y \cdot L_{\alpha}\right)$

for all $y \in U_{0}^{M^{\prime}}$, with $\alpha:=s\left(F_{\bar{\sigma}}\right)+s\left(E_{\bar{\sigma}}\right)$ and $c_{\bar{\sigma}}:=\left\langle F_{\bar{\sigma}}, \mathcal{E}_{\bar{\sigma}}\right\rangle \cdot\left\langle E_{\bar{\sigma}}, \mathcal{F}_{\bar{\sigma}}\right\rangle \neq 0$ : In other words, $\Phi_{\bar{\sigma}}^{\varphi}=\left.c_{\bar{\sigma}}{ }^{-1} \cdot\left(\left(L_{-\alpha} \cdot \mathcal{F}_{\bar{\sigma}}\right) \triangleright S(x) \triangleleft \mathcal{E}_{\bar{\sigma}}\right)\right|_{U_{0}^{M^{\prime}}} \quad($ where $\triangleleft$ and $\triangleright$ denote standard left and right action, cf. [DL], §1.4), hence we have to study $\left\langle S(x), \mathcal{E}_{\bar{\sigma}} \cdot y \cdot L_{-\alpha} \mathcal{F}_{\bar{\sigma}}\right\rangle$ as a function of $y \in U_{\varphi, 0}^{M^{\prime}}$; by linearity we 
can assume $y=L_{\nu}, \quad \nu \in M^{\prime}$. By definition, $\left\langle S(x), \mathcal{E}_{\bar{\sigma}} \cdot y \cdot L_{-\alpha} \mathcal{F}_{\bar{\sigma}}\right\rangle=$ $\left\langle x, S\left(\mathcal{E}_{\bar{\sigma}} \cdot y L_{-\alpha} \cdot \mathcal{F}_{\bar{\sigma}}\right)\right\rangle$; in order to compute the latter we have to "straighten" $S\left(\mathcal{E}_{\bar{\sigma}} \cdot y \cdot L_{-\alpha} \mathcal{F}_{\bar{\sigma}}\right)$, i.e., to express it in terms of a PBW basis of $U_{-} \otimes U_{0}^{M^{\prime}} \otimes U_{+}$. Since $S\left(\mathcal{E}_{\bar{\sigma}} \cdot y \cdot L_{-\alpha} \mathcal{F}_{\bar{\sigma}}\right)=S\left(L_{-\alpha} \mathcal{F}_{\bar{\sigma}}\right) \cdot S(y) \cdot S\left(\mathcal{E}_{\bar{\sigma}}\right)$, let us consider the various factors.

First, $S\left(L_{-\alpha} \mathcal{F}_{\bar{\sigma}}\right) \in U_{<}^{M^{\prime}}$, and $S\left(L_{-\alpha} \mathcal{F}_{\bar{\sigma}}\right)$ does not depend on $y$. Second, $S(y)=S\left(L_{\nu}\right)=L_{-\nu}$. Third, $S\left(\mathcal{E}_{\bar{\sigma}}\right) \in U_{\geq}^{M^{\prime}}$, and $S\left(\mathcal{E}_{\bar{\sigma}}\right)$ does not depend on $y$.

Now we straighten the product. Commuting $S\left(L_{-\alpha} \mathcal{F}_{\bar{\sigma}}\right)$ and $S(y)=$ $L_{-\nu}$ produces a coefficient $q^{-\left(\nu \mid \beta_{\bar{\sigma}}\right)}=\left\langle L_{-\beta_{\bar{\sigma}}}^{\varphi}, L_{\nu}\right\rangle_{\bar{\pi}}$, where $\beta_{\bar{\sigma}} \in Q_{-}$is the weight of $S\left(\mathcal{F}_{\bar{\sigma}}\right)$. Straightening the product $S\left(L_{-\alpha} \mathcal{F}_{\bar{\sigma}}\right) \cdot S\left(\mathcal{E}_{\bar{\sigma}}\right)$ produces a sum $\sum_{k} x_{k}$ of terms which do not depend on $y$. Straightening the product $S(y)=L_{-\nu} \cdot \sum_{k} x_{k}$ produces for each term $x_{k}$ a coefficient $q^{-\left(\nu \mid \gamma_{\bar{\sigma}, k}\right)}=$ $\left\langle L_{-\gamma_{\bar{\sigma}, k}}^{\varphi}, L_{\nu}\right\rangle_{\bar{\pi}}$, where $\gamma_{\bar{\sigma}, k} \in Q_{+}$is the weight of the "positive" part $x_{k}^{+}$of $x_{k}$ (with respect to the triangular decomposition).

Therefore $\left\langle x, S\left(\mathcal{E}_{\bar{\sigma}} \cdot y \cdot L_{-\alpha} \mathcal{F}_{\bar{\sigma}}\right)\right\rangle$ depends on $y$ according to the functions $L_{-\beta_{\bar{\sigma}}}^{\varphi}, L_{-\gamma_{\bar{\sigma}, k}}^{\varphi}$, and $\Phi_{\tau}^{\varphi^{\prime}} \circ S$ : to be precise, $\Phi_{\bar{\sigma}}^{\varphi}=\left.\left(L_{-\alpha} \mathcal{F}_{\bar{\sigma}} \triangleright S(x) \triangleleft \mathcal{E}_{\bar{\sigma}}\right)\right|_{U_{0}^{M^{\prime}}}$ is a linear combination of functions of type $L_{-\beta_{\bar{\sigma}}}^{\varphi} \cdot\left(\Phi_{\tau}^{\varphi^{\prime}} \circ S\right) \cdot L_{-\gamma_{\bar{\sigma}, k}}^{\varphi}=$ $\sum_{\mu \in M} c_{\tau, \mu} L_{-\mu-\beta_{\bar{\sigma}}-\gamma_{\bar{\sigma}, k}}$, so $\Phi_{\bar{\sigma}}^{\varphi} \in U_{\varphi, 0}^{M}$, q.e.d. An entirely analogous procedure - slightly simpler indeed — works for comultiplication, thus proving that $\Delta\left(\Omega_{\varphi}^{M}\right) \subseteq \Omega_{\varphi}^{M} \widehat{\otimes} \Omega_{\varphi}^{M}$. The thesis follows.

Now we introduce integer forms of $U_{q, \varphi}^{M}(\mathfrak{h})$ and prove their first properties. We freely use the term pseudobasis to mean a topological basis of a topological module, so that any element in the module has a unique expansion as a series in the elements of the basis.

Definition 6.5. We define $\mathcal{H}_{\varphi}^{M}$ to be the $k\left[q, q^{-1}\right]$-subalgebra of $U_{q, \varphi}^{M}(\mathfrak{h})$ generated by $\left\{\bar{F}_{\alpha^{r}}^{\varphi}, L_{\mu}^{\varphi}, \bar{E}_{\alpha^{r}}^{\varphi} \mid r=1, \ldots, N ; \mu \in M\right\}$, and $\mathcal{U}_{\varphi}^{M}(\mathfrak{h})$ to be its closure in $U_{q, \varphi}^{M}(\mathfrak{h})$.

Theorem 6.6. $\mathcal{U}_{\varphi}^{M}(\mathfrak{h})$ is a $k\left[q, q^{-1}\right]$-integer form (in topological sense) of $U_{q, \varphi}^{M}(\mathfrak{h})$, as a formal Hopf algebra, with $k\left[q, q^{-1}\right]$-pseudobasis

$(6.2)$

$\widetilde{\mathbb{B}}_{M}^{\varphi}:=\left\{Y_{\eta, \tau, \phi}^{\varphi} \mid \tau \in \mathbb{N}^{n} ; \eta, \phi \in \mathbb{N}^{N}\right\}=\left\{\mathcal{F}_{\eta}^{\varphi} \cdot B_{\eta, \tau, \phi}^{\varphi} \cdot \mathcal{E}_{\phi}^{\varphi} \mid \tau \in \mathbb{N}^{n} ; \eta, \phi \in \mathbb{N}^{N}\right\} ;$

in particular $\nu_{M}^{\varphi}\left(\mathcal{U}_{\varphi}^{M}(\mathfrak{h})\right)=j_{M}\left(\mathfrak{U}_{\varphi}^{M^{\prime}}(\mathfrak{g})^{*}\right)=: \mathfrak{I}_{\varphi}^{M}$.

Proof. By construction $\widetilde{\mathbb{B}}_{M}^{\varphi} \subseteq \mathcal{U}_{\varphi}^{M}(\mathfrak{h})$, so the claim follows from $\S 6.1$ or Remark 6.3. 
Let $\widehat{\Omega}_{\varphi}^{M}:=\Omega_{\varphi}^{M} \cap\left(\nu_{M}^{\varphi}\right)^{-1}\left(\mathcal{I}_{\varphi}^{M}\right)$; notice that (cf. Proposition 5.9(b)) $\widehat{\Omega}_{\varphi}^{M}=\left\{x=\sum_{\sigma} \mathfrak{F}_{\sigma}^{\varphi} \cdot \phi_{\sigma}^{\varphi} \cdot \mathfrak{E}_{\sigma}^{\varphi} \in U_{q, \varphi}^{M}(\mathfrak{h}) \mid \mathfrak{F}_{\sigma}^{\varphi} \in \mathfrak{U}_{\varphi,-}, \phi_{\sigma}^{\varphi} \in \mathfrak{U}_{\varphi, 0}^{M}, \mathfrak{E}_{\sigma}^{\varphi} \in \mathfrak{U}_{\varphi,+}, \forall \sigma\right\}$.

Definition 6.7. We call $\mathfrak{H}_{\varphi}^{M}$ the $k\left[q, q^{-1}\right]$-subalgebra of $U_{q, \varphi}^{M}(\mathfrak{h})$ generated by $\left\{\left(F_{i}^{\varphi}\right)^{(f)},\left(\begin{array}{c}M_{i}^{\varphi} ; c \\ t\end{array}\right),\left(M_{i}^{\varphi}\right)^{-1},\left(E_{i}^{\varphi}\right)^{(e)} \mid f, c, t, e \in \mathbb{N} ; i=1, \ldots, n\right\}$, and $\mathfrak{U}_{\varphi}^{M}(\mathfrak{h})$ the set

$$
\begin{aligned}
\left\{x \in \widehat{\Omega}_{\varphi}^{M} \mid x\right. & =\sum_{n=0}^{+\infty} x_{n}, \\
x_{n} & \left.\in \sum_{\sum_{h}\left(a_{h}+b_{h}\right)=n} \prod_{h=1}^{N} \prod_{r, s=1}^{a_{h}, b_{h}}\left(q^{r}-q^{-r}\right) \cdot\left(q^{s}-q^{-s}\right) \cdot \mathfrak{H}_{\varphi}^{M} \forall n\right\} .
\end{aligned}
$$

Theorem 6.8. $\mathfrak{U}_{\varphi}^{M}(\mathfrak{h})$ is a $k\left[q, q^{-1}\right]$-integer form of $U_{q, \varphi}^{M}(\mathfrak{h})$ and $\Omega_{\varphi}^{M}$. Proof. By construction $\mathfrak{U}_{\varphi}^{M}(\mathfrak{h})$ is a $k\left[q, q^{-1}\right]$-subalgebra of $U_{q, \varphi}^{M}(\mathfrak{h})$ and $\Omega_{\varphi}^{M}$; moreover Theorem 6.2 and Proposition 5.9(b) ensure that $\widehat{\Omega}_{\varphi}^{M}$ is a $k\left[q, q^{-1}\right]$ integer form (in topological sense) of $\Omega_{\varphi}^{M}$ (as an algebra), hence also $\mathfrak{U}_{\varphi}^{M}(\mathfrak{h})$ is. Proposition 5.9(b) and Lemma 6.4 imply that $\widehat{\Omega}_{\varphi}^{M}$ is a formal Hopf subalgebra of $\Omega_{\varphi}^{M}$. Finally the analysis in $\S 5.16$ (especially (5.8) and (5.9)) via $\nu_{M}{ }^{-1}$ gives $S\left(\mathfrak{U}_{\varphi}^{M}(\mathfrak{h})\right)=\mathfrak{U}_{\varphi}^{M}(\mathfrak{h})$ and $\Delta\left(\mathfrak{U}_{\varphi}^{M}(\mathfrak{h})\right) \subseteq \mathfrak{U}_{\varphi}^{M}(\mathfrak{h}) \widehat{\otimes} \mathfrak{U}_{\varphi}^{M}(\mathfrak{h})$.

6.9. Presentation of $\mathfrak{U}_{\varphi}^{M}(\mathfrak{h})$. By the similar result available for $\mathfrak{U}_{\varphi}^{M}(\mathfrak{g}) \cong$ $\mathfrak{U}_{q, 0}^{M}(\mathfrak{g}) \quad(\mathrm{cf} . \quad[\mathbf{D L}], \S 3.4)$ we get a presentation of $\mathfrak{U}_{\varphi}^{M}(\mathfrak{h})$ by (topological) generators and relations. The algebra $\mathfrak{H}_{\varphi}^{M}$ of $\S 6.7$ is the associative $k\left[q, q^{-1}\right]$ algebra with 1 with generators

$$
M_{i}^{\varphi},\left(M_{i}^{\varphi}\right)^{-1},\left(\begin{array}{c}
M_{i}^{\varphi} ; c \\
t
\end{array}\right),\left(E_{i}^{\varphi}\right)^{(r)}\left(F_{i}^{\varphi}\right)^{(s)}
$$

$\left(i=1, \ldots, n ; c \in \mathbb{Z}, t, r, s \in \mathbb{N}\right.$; here we set $\left.M_{i}^{\varphi}:=L_{\mu_{i}}^{\varphi}\right)$, and relations

$$
\begin{gathered}
M_{i}^{\varphi}\left(M_{i}^{\varphi}\right)^{-1}=1=\left(M_{i}^{\varphi}\right)^{-1} M_{i}^{\varphi}, \quad\left(M_{i}^{\varphi}\right)^{ \pm 1}\left(M_{j}^{\varphi}\right)^{ \pm 1}=\left(M_{j}^{\varphi}\right)^{ \pm 1}\left(M_{i}^{\varphi}\right)^{ \pm 1} \\
\left(M_{i}^{\varphi}\right)^{ \pm 1}\left(\begin{array}{c}
M_{j}^{\varphi} ; c \\
t
\end{array}\right)=\left(\begin{array}{c}
M_{j}^{\varphi} ; c \\
t
\end{array}\right)\left(M_{i}^{\varphi}\right)^{ \pm 1}, \\
\left(\begin{array}{c}
M_{i}^{\varphi} ; c \\
0
\end{array}\right)=0, \quad\left(q_{i}-1\right)\left(\begin{array}{c}
M_{i}^{\varphi} ; 0 \\
1
\end{array}\right)=M_{i}^{\varphi}-1 \\
\left(\begin{array}{c}
M_{i}^{\varphi} ; c \\
t
\end{array}\right)\left(\begin{array}{c}
M_{i}^{\varphi} ; c-t \\
s
\end{array}\right)=\left(\begin{array}{c}
t+s \\
t
\end{array}\right)_{q}\left(\begin{array}{c}
M_{i}^{\varphi} ; c \\
t+s
\end{array}\right), \quad \forall t, s
\end{gathered}
$$




$$
\begin{aligned}
& \left(\begin{array}{c}
M_{i}^{\varphi} ; c+1 \\
t
\end{array}\right)-q^{t}\left(\begin{array}{c}
M_{i}^{\varphi} ; c \\
t
\end{array}\right)=\left(\begin{array}{c}
M_{i}^{\varphi} ; c \\
t-1
\end{array}\right), \quad \forall t \geq 1 \\
& \left(\begin{array}{c}
M_{i}^{\varphi} ; c \\
t
\end{array}\right)=\sum_{p \geq 0}^{p \leq c, t} q^{(c-p)(t-p)}\left(\begin{array}{c}
c \\
p
\end{array}\right)_{q}\left(\begin{array}{c}
M_{i}^{\varphi} ; 0 \\
t-1
\end{array}\right), \quad \forall c \geq 0 \\
& \left(\begin{array}{c}
M_{i}^{\varphi} ;-c \\
t
\end{array}\right)=\sum_{p=0}^{t}(-1)^{p} q^{-t(c+p)+p(p+1) / 2}\left(\begin{array}{c}
p+c-1 \\
p
\end{array}\right)_{q}\left(\begin{array}{c}
M_{i}^{\varphi} ; 0 \\
t-p
\end{array}\right), \quad \forall c \geq 1 \\
& \left(\begin{array}{c}
M_{i}^{\varphi} ; c+1 \\
t
\end{array}\right)-\left(\begin{array}{c}
M_{i}^{\varphi} ; c \\
t
\end{array}\right)=q^{c-t+1} M_{i}^{\varphi}\left(\begin{array}{c}
M_{i}^{\varphi} ; c \\
t-1
\end{array}\right), \quad \forall t \geq 1 \\
& M_{i}^{\varphi}\left(E_{j}^{\varphi}\right)^{(p)}=q^{p\left(\alpha_{j} \mid(1+\varphi)\left(\mu_{i}\right)\right)}\left(E_{j}^{\varphi}\right)^{(p)} M_{i}^{\varphi}, \\
& M_{i}^{\varphi}\left(F_{j}^{\varphi}\right)^{(p)}=q^{p\left(\alpha_{j} \mid(1-\varphi)\left(\mu_{i}\right)\right)}\left(F_{j}^{\varphi}\right)^{(p)} M_{i}^{\varphi} \\
& \left(\begin{array}{c}
M_{i}^{\varphi} ; c \\
t
\end{array}\right)\left(E_{j}^{\varphi}\right)^{(p)}=\left(E_{j}^{\varphi}\right)^{(p)}\left(\begin{array}{c}
M_{i}^{\varphi} ; c+p\left(\alpha_{j} \mid(1+\varphi)\left(\mu_{i}\right)\right) \\
t
\end{array}\right) \\
& \left(\begin{array}{c}
M_{i}^{\varphi} ; c \\
t
\end{array}\right)\left(F_{j}^{\varphi}\right)^{(p)}=\left(F_{j}^{\varphi}\right)^{(p)}\left(\begin{array}{c}
M_{i}^{\varphi} ; c+p\left(\alpha_{j} \mid(1-\varphi)\left(\mu_{i}\right)\right) \\
t
\end{array}\right) \\
& \left(E_{i}^{\varphi}\right)^{(r)}\left(E_{i}^{\varphi}\right)^{(s)}=\left[\begin{array}{c}
r+s \\
r
\end{array}\right]_{q_{i}}\left(E_{i}^{\varphi}\right)^{(r+s)},\left(F_{i}^{\varphi}\right)^{(r)}\left(F_{i}^{\varphi}\right)^{(s)}=\left[\begin{array}{c}
r+s \\
r
\end{array}\right]_{q_{i}}\left(F_{i}^{\varphi}\right)^{(r+s)} \\
& \sum_{r+s=1-a_{i j}}(-1)^{s}\left(E_{i}^{\varphi}\right)^{(r)} E_{j}^{\varphi}\left(E_{i}^{\varphi}\right)^{(s)}=0, \quad \forall i \neq j \\
& \sum_{r+s=1-a_{i j}}(-1)^{s}\left(F_{i}^{\varphi}\right)^{(r)} F_{j}^{\varphi}\left(F_{i}^{\varphi}\right)^{(s)}=0, \quad \forall i \neq j \\
& \left(E_{i}^{\varphi}\right)^{(0)}=1, \quad\left(E_{i}^{\varphi}\right)^{(r)}\left(F_{j}^{\varphi}\right)^{(s)}=\left(F_{j}^{\varphi}\right)^{(s)}\left(E_{i}^{\varphi}\right)^{(r)}, \quad\left(F_{i}^{\varphi}\right)^{(0)}=1 .
\end{aligned}
$$

Then $\mathfrak{U}_{\varphi}^{M}(\mathfrak{h})$ is the completion of $\mathfrak{H}_{\varphi}^{M}$ obtained by taking formal series in the PBW monomials of $\mathfrak{U}_{\varphi,-}$ and $\mathfrak{U}_{\varphi,+}$, with coefficients in $\mathfrak{U}_{\varphi, 0}^{M}$, which satisfy the condition in (6.3). Finally, formulas in $\S 5.16$ yield - via $\nu_{M}^{\varphi}-$ the following (where $K_{i}^{\varphi}:=L_{\alpha_{i}}^{\varphi}$ ):

$$
\begin{aligned}
& \Delta\left(F_{i}^{\varphi}\right) \equiv F_{i}^{\varphi} \otimes 1+1 \otimes F_{i}^{\varphi}+\left(q_{i}-1\right) \cdot\left(\begin{array}{c}
K_{i}^{\varphi} ; 0 \\
1
\end{array}\right) \otimes F_{i}^{\varphi}+\left(q_{i}-q_{i}^{-1}\right)^{-1} \\
& \quad \sum_{\alpha, \beta \in R^{+}} C_{\alpha, \beta}^{i,+}\left(q_{\alpha}-q_{\alpha}^{-1}\right)\left(q_{\beta}-q_{\beta}^{-1}\right) K_{i}^{\varphi} E_{\alpha}^{\varphi} \otimes F_{\beta}^{\varphi} \\
& \Delta\left(\left(\begin{array}{c}
M_{i}^{\varphi} ; 0 \\
1
\end{array}\right)\right) \equiv\left(\begin{array}{c}
M_{i}^{\varphi} ; 0 \\
1
\end{array}\right) \otimes 1+1 \otimes\left(\begin{array}{c}
M_{i}^{\varphi} ; 0 \\
1
\end{array}\right) \\
& \quad+\left(q_{i}-1\right) \cdot\left(\begin{array}{c}
M_{i}^{\varphi} ; 0 \\
1
\end{array}\right) \otimes\left(\begin{array}{c}
M_{i}^{\varphi} ; 0 \\
1
\end{array}\right)+(2)_{q^{-1}}^{2}\left(d_{i}\right)_{q}^{-1}
\end{aligned}
$$


$\cdot \sum_{\gamma \in R^{+}}(q-1)\left[d_{\gamma}\right]_{q}\left[\left(\mu_{i} \mid \gamma\right)\right]_{q} \cdot M_{i}^{\varphi} E_{\gamma}^{\varphi} \otimes F_{\gamma}^{\varphi} M_{i}^{\varphi} \quad \bmod \left(q-q^{-1}\right)^{2}$ $\Delta\left(E_{i}^{\varphi}\right) \equiv 1 \otimes E_{i}^{\varphi}+E_{i}^{\varphi} \otimes 1+\left(q_{i}-1\right) \cdot E_{i}^{\varphi} \otimes\left(\begin{array}{c}K_{i}^{\varphi} ; 0 \\ 1\end{array}\right)-\left(q_{i}-q_{i}^{-1}\right)^{-1}$ $\sum_{\alpha, \beta \in R^{+}} C_{\alpha, \beta}^{i,-}\left(q_{\alpha}-q_{\alpha}^{-1}\right)\left(q_{\beta}-q_{\beta}^{-1}\right) E_{\alpha}^{\varphi} \otimes F_{\beta}^{\varphi} K_{i}^{\varphi} \quad \bmod \left(q-q^{-1}\right)^{2}$ $S\left(F_{i}^{\varphi}\right) \equiv-q_{i}^{-2} \cdot F_{i}^{\varphi}\left(K_{i}^{\varphi}\right)^{-1}, S\left(E_{i}^{\varphi}\right) \equiv-q_{i}^{+2} \cdot\left(K_{i}^{\varphi}\right)^{-1} E_{i}^{\varphi} \quad \bmod \left(q-q^{-1}\right)$ $S\left(\left(\begin{array}{c}M_{i}^{\varphi} ; 0 \\ 1\end{array}\right)\right) \equiv-\left(M_{i}^{\varphi}\right)^{-1} \cdot\left(\begin{array}{c}M_{i}^{\varphi} ; 0 \\ 1\end{array}\right) \quad \bmod \left(q-q^{-1}\right)$ $\epsilon\left(F_{i}^{\varphi}\right)=0, \quad \epsilon\left(\left(\begin{array}{c}M_{i}^{\varphi} ; 0 \\ 1\end{array}\right)\right)=0, \quad \epsilon\left(E_{i}^{\varphi}\right)=0$.

Definition 6.10. We call $\xi_{M}^{\varphi}$ the embedding of formal Hopf algebras

$$
\xi_{M}^{\varphi}:=\left(\nu_{M}^{\varphi}\right)^{-1} \circ \mu_{M}^{\varphi}: F_{q, \varphi}^{M}[G] \longleftrightarrow U_{q, \varphi}^{M}(\mathfrak{h}) .
$$

Theorem 6.11. The embedding $\xi_{M}^{\varphi}: F_{q, \varphi}^{M}[G] \longleftrightarrow U_{q, \varphi}^{M}(\mathfrak{h})$ induces algebra monomorphisms $\xi_{M}^{\varphi}: F_{q, \varphi}^{M}[G] \longleftrightarrow \mathbf{H}_{\varphi}^{M}, \quad \xi_{M}^{\varphi}: \mathfrak{F}_{\varphi}^{M}[G] \quad \longleftrightarrow \mathcal{H}_{\varphi}^{M}$, $\xi_{M}^{\varphi}: \mathcal{F}_{\varphi}^{M}[G] \longleftrightarrow \mathfrak{H}_{\varphi}^{M}$ and algebra isomorphisms $\xi_{M}^{\varphi}: F_{q, \varphi}^{M}[G]\left[\psi_{-\rho}^{-1}\right] \stackrel{\cong}{\longrightarrow} \mathbf{H}_{\varphi}^{M}$, $\xi_{M}^{\varphi}: \mathfrak{F}_{\varphi}^{M}[G]\left[\psi_{-\rho}^{-1}\right] \stackrel{\cong}{\longrightarrow} \mathcal{H}_{\varphi}^{M}, \xi_{M}^{\varphi}: \mathcal{F}_{\varphi}^{M}[G]\left[\psi_{-\rho}^{-1}\right] \stackrel{\cong}{\longrightarrow} \mathfrak{H}_{\varphi}^{M}$ whose images are dense respectively in $U_{q, \varphi}^{M}(\mathfrak{h})$, in $\mathcal{U}_{\varphi}^{M}(\mathfrak{h})$, in $\mathfrak{U}_{\varphi}^{M}(\mathfrak{h})$.

6.12. Quantum Poisson pairing. In this section we define perfect Hopf pairings $U_{q, \varphi}^{M}(\mathfrak{h}) \otimes U_{q, \varphi}^{M^{\prime}}(\mathfrak{g}) \longrightarrow k(q)$ which provide quantizations of the Hopf pairings $F\left[G^{\tau}\right] \otimes U\left(\mathfrak{g}^{\tau}\right) \rightarrow k\left(\right.$ or $\left.F^{\infty}\left[G^{\tau}\right] \otimes U\left(\mathfrak{g}^{\tau}\right) \rightarrow k\right)$ and $U\left(\mathfrak{h}^{\tau}\right) \otimes$ $F\left[H^{\tau}\right] \rightarrow k$ and of the Lie bialgebra pairing $\mathfrak{h}^{\tau} \otimes \mathfrak{g}^{\tau} \rightarrow k$ : Therefore we call them "(multiparameter) quantum Poisson pairings"; moreover they also provide new interesting pairings between function algebras. Since $j_{M}{ }^{-1} \circ \nu_{M}^{\varphi}$ : $U_{q, \varphi}^{M}(\mathfrak{h}) \stackrel{\cong}{\longrightarrow} U_{q, \varphi}^{M^{\prime}}(\mathfrak{g})^{*}$, evaluation gives a perfect Hopf pairing

$$
\pi_{q, \varphi}^{M}: U_{q, \varphi}^{M}(\mathfrak{h}) \otimes U_{q, \varphi}^{M^{\prime}}(\mathfrak{g}) \longrightarrow k(q)
$$

defined by $\pi_{q, \varphi}^{M}(h, g):=\left\langle j_{M}{ }^{-1}\left(\nu_{M}^{\varphi}(h)\right), g\right\rangle$ for all $h \in U_{q, \varphi}^{M}(\mathfrak{h}), g \in U_{q, \varphi}^{M^{\prime}}(\mathfrak{g})$.

We call $\pi_{q, \varphi}^{M}$ (multiparameter) quantum Poisson pairing.

By previous analysis, the integer forms of quantum enveloping algebras are $k\left[q, q^{-1}\right]$-dual of each other (cf. $\left.\S 2.6\right)$ with respect to $\pi_{q, \varphi}^{M}$; so the latter restrict to perfect pairings $\pi_{q, H_{M}^{\tau}}^{\varphi}: \mathfrak{U}_{\varphi}^{M^{\prime}}(\mathfrak{h}) \otimes \mathcal{U}_{\varphi}^{M}(\mathfrak{g}) \longrightarrow k\left[q, q^{-1}\right], \pi_{q, G_{M}^{\tau}}^{\varphi}: \mathcal{U}_{\varphi}^{M}(\mathfrak{h}) \otimes \mathfrak{U}_{\varphi}^{M^{\prime}}(\mathfrak{g}) \longrightarrow k\left[q, q^{-1}\right] ;$ same symbols will also denote the Hopf pairings $\pi_{q, H_{M}^{\tau}}^{\varphi}: \mathcal{F}_{\varphi}^{M^{\prime}}[G] \otimes \mathcal{U}_{\varphi}^{M}(\mathfrak{g}) \longrightarrow$ $k\left[q, q^{-1}\right]$, resp. $\pi_{q, G_{M}^{\tau}}^{\varphi}: \mathfrak{F}_{\varphi}^{M}[G] \otimes \mathfrak{U}_{\varphi}^{M^{\prime}}(\mathfrak{g}) \longrightarrow k\left[q, q^{-1}\right]$, got by restriction of 
the previous ones: Hereafter we identify $F_{q, \varphi}^{M}[G]$ with its image in $U_{q, \varphi}^{M}(\mathfrak{h})$ via $\xi_{M}^{\varphi}$, and similarly for integer forms.

\section{Specialization at roots of 1 .}

7.1. The case $q \rightarrow 1$ : Specialization of $\mathfrak{U}_{\varphi}^{M}(\mathfrak{h})$ to $U\left(\mathfrak{h}^{\tau}\right)$ and consequences. Recall (cf. §2.1) that $\tau=\left(\tau_{1}, \ldots, \tau_{n}\right):=\frac{1}{2}\left(\varphi\left(\alpha_{1}\right), \ldots, \varphi\left(\alpha_{n}\right)\right)$. Now set

$$
\mathfrak{U}_{1, \varphi}^{M}(\mathfrak{h}):=\mathfrak{U}_{\varphi}^{M}(\mathfrak{h}) /(q-1) \mathfrak{U}_{\varphi}^{M}(\mathfrak{h}) \cong \mathfrak{U}_{\varphi}^{M}(\mathfrak{h}) \otimes_{k\left[q, q^{-1}\right]} k
$$

let $p_{1}^{\varphi}: \mathfrak{U}_{\varphi}^{M}(\mathfrak{h}) \rightarrow \mathfrak{U}_{1, \varphi}^{M}(\mathfrak{h})$ be the canonical projection, and set $\mathrm{f}_{i}^{\tau}:=$ $p_{1}^{\varphi}\left(\left(F_{i}^{\varphi}\right)^{(1)}\right), \mathrm{m}_{i}^{\tau}:=p_{1}^{\varphi}\left(\left(\begin{array}{c}M_{i}^{\varphi} ; 0 \\ 1\end{array}\right)\right), \mathrm{e}_{i}^{\tau}:=p_{1}^{\varphi}\left(\left(E_{i}^{\varphi}\right)^{(1)}\right),\left(\right.$ where $\left.M_{i}:=L_{\mu_{i}}^{\varphi}\right)$ for all $i=1, \ldots, n$.

Theorem 7.2. For $q \rightarrow 1, \mathfrak{U}_{\varphi}^{M}(\mathfrak{h})$ specializes to the Poisson Hopf coalgebra $U\left(\mathfrak{h}^{\tau}\right)$; in other words, there exists an isomorphism of Poisson Hopf coalgebras

$$
\mathfrak{U}_{1, \varphi}^{M}(\mathfrak{h}) \cong U\left(\mathfrak{h}^{\tau}\right)
$$

Proof. The proof mimick that for $\mathfrak{U}_{1, \varphi}^{M}(\mathfrak{g}) \cong U\left(\mathfrak{g}^{\tau}\right)$. From the presentation of $\mathfrak{U}_{\varphi}^{M}(\mathfrak{h})$ we get $\mathfrak{U}_{1, \varphi}^{M}(\mathfrak{h})=\left.\mathfrak{H}_{\varphi}^{M}\right|_{q=1}:=\mathfrak{H}_{\varphi}^{M} /(q-1) \mathfrak{H}_{\varphi}^{M}$, hence we are reduced to study $\left.\mathfrak{H}_{\varphi}^{M}\right|_{q=1}$; moreover the presentation of $\mathfrak{H}_{\varphi}^{M}$ provides one of $\left.\mathfrak{H}_{\varphi}^{M}\right|_{q=1}$. Now the definition of $\left.\mathfrak{H}_{\varphi}^{M}\right|_{q=1}$ and the explicit form of the PBW basis of $\mathfrak{U}_{\varphi, 0}^{M}$ (cf. $\left.\S 2.5\right)$ imply that the elements $\left(F_{i}^{\varphi}\right)^{(r)},\left(\begin{array}{c}M_{i}^{\varphi} ; 0 \\ t\end{array}\right),\left(M_{i}^{\varphi}\right)^{-1}$, $\left(E_{i}^{\varphi}\right)^{(s)}(i=1, \ldots, n ; r, t, s \in \mathbb{N})$ are enough to generate $\mathfrak{H}_{\varphi}^{M}$; finally, straightforward computations give $p_{1}^{\varphi}\left(\left(F_{i}^{\varphi}\right)^{(r)}\right)=\frac{\left(\mathrm{f}_{i}^{\tau}\right)^{r}}{r !}, p_{1}^{\varphi}\left(\left(\begin{array}{c}M_{i}^{\varphi} ; 0 \\ t\end{array}\right)\right)=$ $\left(\begin{array}{c}\mathrm{m}_{i}^{\tau} \\ t\end{array}\right), \quad p_{1}^{\varphi}\left(\left(M_{i}^{\varphi}\right)^{-1}\right)=1, \quad p_{1}^{\varphi}\left(\left(E_{i}^{\varphi}\right)^{(s)}\right)=\frac{\left(\mathrm{e}_{i}^{\tau}\right)^{s}}{s !} \quad\left(\right.$ where $\quad\left(\begin{array}{c}\mathrm{m}_{i}^{\varphi} \\ t\end{array}\right):=$ $\left.\frac{\mathrm{m}_{i}^{\varphi}\left(\mathrm{m}_{i}^{\varphi}-1\right)\left(\mathrm{m}_{i}^{\varphi}-2\right) \cdots\left(\mathrm{m}_{i}^{\varphi}-t+1\right)}{t !}\right)$, hence $\mathfrak{U}_{1, \varphi}^{M}(\mathfrak{h})=\left.\mathfrak{H}_{\varphi}^{M}\right|_{q=1}$ is generated by the $\mathrm{f}_{i}^{\tau}$ 's, $\mathrm{m}_{i}^{\tau}$ 's, $\mathrm{e}_{i}^{\tau}$ 's, with some relations.

When $M=Q$ this presentation is exactly the same of $U\left(\mathfrak{h}^{\tau}\right)$ (cf. (1.2)), with $\mathrm{h}_{i}^{\tau}=\mathrm{m}_{i}^{\tau}$; comparing (1.3) with formulas in $\S 6.9$ (for $q=1$ ) shows that also the Hopf structure is the same. In particular $\mathfrak{U}_{1, \varphi}^{Q}(\mathfrak{h})$ is cocommutative, hence has a canonical co-Poisson structure given by $\delta:=\left.\left(\frac{\Delta-\Delta^{o p}}{q-1}\right)\right|_{q=1}$, described by formulas - deduced from those in $\S 6.9$ - which do coincide with (1.4), as a straightforward checking shows.

Finally, for $M \neq Q$ we prove that $\mathfrak{U}_{1, \varphi}^{M}(\mathfrak{h}) \cong \mathfrak{U}_{1, \varphi}^{Q}(\mathfrak{h})$ as Poisson Hopf coalgebras: Since $\mathfrak{U}_{\varphi}^{M}(\mathfrak{h}) \supseteq \mathfrak{U}_{\varphi}^{Q}(\mathfrak{h})$ by definition, it is enough to check that 
$\left.\mathfrak{H}_{\varphi}^{M}\right|_{q=1}=\left.\mathfrak{H}_{\varphi}^{Q}\right|_{q=1}$ as $k$-vector spaces. Assume we are in the simply laced case. Since $M_{i}^{\varphi}:=L_{\mu_{i}}^{\varphi}$ and $K_{j}^{\varphi}:=L_{\alpha_{j}}^{\varphi}$, it is $K_{j}^{\varphi}:=\prod_{i=1}^{n}\left(M_{\alpha_{i}}^{\varphi}\right)^{c_{i j}}$, where $c_{i j} \in \mathbb{Z}$ are such that $\alpha_{j}=\sum_{i=1}^{n} c_{i j} \mu_{i}$. Then $\left.\left(\begin{array}{c}K_{j}^{\varphi} ; 0 \\ t\end{array}\right)\right|_{q=1}=\sum_{i=1}^{n} c_{i j}$. $\left.\left(\begin{array}{c}M_{i}^{\varphi} ; 0 \\ t\end{array}\right)\right|_{q=1}$ so that $\left.\mathfrak{U}_{\varphi, 0}^{M}\right|_{q=1}=\left.\mathfrak{U}_{\varphi, 0}^{Q}\right|_{q=1}$ follows, whence $\left.\mathfrak{H}_{\varphi}^{M}\right|_{q=1}=\left.\mathfrak{H}_{\varphi}^{Q}\right|_{q=1}$, q.e.d. In the other cases $M=P$, and this argument still works, mutatis mutandis, because $\alpha_{j}=\sum_{i=1}^{n} a_{i j} \omega_{i}$, hence $K_{j}^{\varphi}:=\prod_{i=1}^{n}\left(L_{\alpha_{i}}^{\varphi}\right)^{a_{i j}}$, so that $\left.\left(\begin{array}{c}K_{j}^{\varphi} ; 0 \\ t\end{array}\right)\right|_{q=1}=\left.\sum_{i=1}^{n} a_{i j} \cdot\left(\begin{array}{c}L_{i}^{\varphi} ; 0 \\ t\end{array}\right)\right|_{q=1}$ and we are done again.

Remark. Thus $\mathfrak{U}_{\varphi}^{M}(\mathfrak{h})$ provides the announced infinitesimal quantization of $H^{\tau}$. This can be partially explained as follows. $\mathfrak{U}_{\varphi}^{M}(\mathfrak{h})$ is a subspace of $U_{q, \varphi}^{M^{\prime}}(\mathfrak{g}){ }^{*}$ made of series satisfying a certain "growth condition" (cf. (6.3)): then specializing $q$ at 1 one gets an isomorphism of Hopf algebras $\mathfrak{U}_{1, \varphi}^{M}(\mathfrak{h}) \cong$ $\left\{f \in F\left[H_{M}^{\tau}\right]^{*} \mid \exists n \in \mathbb{N}: f\left(\mathfrak{e}^{n}\right)=0\right\}$ where $\mathfrak{e}:=\operatorname{Ker}\left(\epsilon: F\left[H_{M}^{\tau}\right] \rightarrow k\right)$, and $\mathfrak{e}=\mathfrak{m}_{e}$, where $\mathfrak{m}_{e}$ is the maximal ideal of $F\left[H_{M}^{\tau}\right]$ associated to $e \in H_{M}^{\tau}$. Since $\left\{f \in F\left[H_{M}^{\tau}\right]^{*} \mid \exists n \in \mathbb{N}: f\left(\mathfrak{m}_{e}^{n}\right)=0\right\} \cong U\left(\mathfrak{h}^{\tau}\right)$ as Hopf algebras, we conclude that there exists a Hopf algebra isomorphism $\mathfrak{U}_{1, \varphi}^{M}(\mathfrak{h}) \cong U\left(\mathfrak{h}^{\tau}\right)$. But regarding co-Poisson structure, such an analysis gives no information, thus the proof of Theorem 7.2 given above is really necessary.

The previous theorem has two interesting consequences. As for the first, set

$$
\mathcal{F}_{1, \varphi}^{M}[G]:=\mathcal{F}_{\varphi}^{M}[G] /(q-1) \mathcal{F}_{\varphi}^{M}[G] \cong \mathcal{F}_{\varphi}^{M}[G] \otimes_{k\left[q, q^{-1}\right]} k
$$

Theorem 7.3. The Hopf algebra $\mathcal{F}_{\varphi}^{M}[G]$ specializes to the Poisson Hopf coalgebra $U\left(\mathfrak{h}^{\tau}\right)$ for $q \rightarrow 1$; in other words, there exists an isomorphism of Poisson Hopf coalgebras

$$
\mathcal{F}_{1, \varphi}^{M}[G] \cong U\left(\mathfrak{h}^{\tau}\right)
$$

Proof. Consider the monomorphism $\xi_{M}^{\varphi}: \mathcal{F}_{\varphi}^{M}[G] \smile \mathfrak{U}_{\varphi}^{M}(\mathfrak{h})$ and compare it with the isomorphism $\xi_{M}^{\varphi}: \mathcal{F}_{\varphi}^{M}[G]\left[\psi_{-\rho}^{-1}\right] \stackrel{\cong}{\longrightarrow} \mathfrak{H}_{\varphi}^{M} \subseteq \mathfrak{U}_{\varphi}^{M}(\mathfrak{h})$. When $q \rightarrow 1$ we get $\mathfrak{U}_{1, \varphi}^{M}(\mathfrak{h})=\left.\mathfrak{H}_{\varphi}^{M}\right|_{q=1}=\left.\left(\mathcal{F}_{\varphi}^{M}[G]\left[\psi_{-\rho}^{-1}\right]\right)\right|_{q=1}=\mathcal{F}_{1, \varphi}^{M}[G]\left[\left.\psi_{-\rho}^{-1}\right|_{q=1}\right] ;$ but $\xi_{M}^{\varphi}\left(\psi_{-\rho}^{-1}\right)=L_{\rho}^{\varphi}=\prod_{i=1}^{n} M_{i}^{\varphi}$ (cf. Theorem 5.14), hence $\left.\xi_{M}^{\varphi}\left(\psi_{-\rho}^{-1}\right)\right|_{q=1}=$ $\left.\prod_{i=1}^{n} M_{i}^{\varphi}\right|_{q=1}=1$ because $M_{i}^{\varphi}=1+\left(q_{i}-1\right) \cdot\left(\begin{array}{c}M_{i}^{\varphi} ; 0 \\ 1\end{array}\right) \equiv 1 \quad \bmod (q-1)$. Therefore $\mathfrak{U}_{1, \varphi}^{M}(\mathfrak{h}) \cong \mathcal{F}_{1, \varphi}^{M}[G]\left[\left.\psi_{-\rho}^{-1}\right|_{q=1}\right]=\mathcal{F}_{1, \varphi}^{M}[G]$, whence the thesis. 
Remark. Thus $F_{q, \varphi}^{M}[G]$ too yields an infinitesimal quantization of $H^{\tau}$; compared with $U_{q, \varphi}^{M}(\mathfrak{h})$ the advantage is that $F_{q, \varphi}^{M}[G]$ is a usual Hopf algebra, whereas $U_{q, \varphi}^{M}(\mathfrak{h})\left(\right.$ or $\left.\mathfrak{U}_{\varphi}^{M}(\mathfrak{h})\right)$ is a topological Hopf algebra. Furthermore, for the classical groups there exists a presentation of $F_{q, 0}^{P}[G]$ by generators and relations, hence - at least in principle - one can study $\mathcal{F}_{\varphi, 0}^{P}[G]$ exploiting such a presentation. For $G=S L(n+1)$ this is done in $[\mathbf{G a}]$.

Theorem 7.3 gives $\mathcal{F}_{\varphi}^{M}[G] \stackrel{q \rightarrow 1}{\longrightarrow} U\left(\mathfrak{h}^{\tau}\right)$, which is the dual result (in the sense of Poisson duality) of $\mathcal{U}_{\varphi}^{M^{\prime}}(\mathfrak{g}) \stackrel{q \rightarrow 1}{\longrightarrow} F\left[H_{M}^{\tau}\right]$. The original proof of the latter result in $[\mathbf{D K P}]$ (see also $[\mathbf{D P}]$ ) is lenghty involved and complicated, requiring very hard computations; on the contrary, we can deduce it as an easy consequence of Theorem 7.2:

Theorem 7.4. The Hopf algebra $\mathcal{U}_{\varphi}^{M}(\mathfrak{g})$ specializes to the Poisson Hopf algebra $F\left[H_{M}^{\tau}\right]$ for $q \rightarrow 1$; in other words, there exists an isomorphism of Poisson Hopf algebras

$$
\mathcal{U}_{1, \varphi}^{P}(\mathfrak{g}):=\mathcal{U}_{\varphi}^{M}(\mathfrak{g}) /(q-1) \mathcal{U}_{\varphi}^{M}(\mathfrak{g}) \cong F\left[H_{M}^{\tau}\right] .
$$

Proof. Since $\mathcal{U}_{\varphi}^{M}(\mathfrak{g})$ is perfectly paired with $\mathfrak{U}_{\varphi}^{M^{\prime}}(\mathfrak{h})$, we have that $\mathcal{U}_{1, \varphi}^{M}(\mathfrak{g})$ is perfectly paired with $\mathfrak{U}_{1, \varphi}^{M^{\prime}}(\mathfrak{h}) \cong U\left(\mathfrak{h}^{\tau}\right)$ : The latter is cocommutative, hence the former is commutative. Then $\mathcal{U}_{1, \varphi}^{M}(\mathfrak{g})$ is a finitely generated commutative Hopf algebra over $k$, hence it is the algebra of (regular) functions of an affine algebraic group, say $H^{\prime}$; moreover $\mathcal{U}_{1, \varphi}^{M}(\mathfrak{g})=F\left[H^{\prime}\right]$ inherits from $\mathcal{U}_{\varphi}^{M}(\mathfrak{g})$ a Poisson structure, so $H^{\prime}$ is a Poisson group. Like in $[\mathbf{D P}]$ it is clear from the presentation of $\mathcal{U}_{\varphi}^{M}(\mathfrak{g})$ that $F\left[H^{\prime}\right]\left(=\mathcal{U}_{1, \varphi}^{M}(\mathfrak{g})\right) \cong F\left[H_{M}^{\tau}\right]$ as Hopf algebras, hence $H^{\prime}=H_{M}^{\tau}$ as algebraic groups (the non-trivial part in $[\mathbf{D P}]$ is that dealing with Poisson structures). Now the Hopf pairing among $\mathfrak{U}_{1, \varphi}^{M^{\prime}}(\mathfrak{h}) \cong U\left(\mathfrak{h}^{\tau}\right)$ and $\mathcal{U}_{1, \varphi}^{M}(\mathfrak{g})=F\left[H^{\prime}\right]=F\left[H_{M}^{\tau}\right]$ is compatible with Poisson and co-Poisson structures, that is $\langle h,\{f, g\}\rangle=\langle\delta(h), f \otimes g\rangle$, where $\delta$ is the Poisson cobracket of $\mathfrak{U}_{1, \varphi}^{M^{\prime}}(\mathfrak{h})=U\left(\mathfrak{h}^{\tau}\right)$ and $\{$,$\} is either the Poisson$ bracket $\{,\}_{\star}$ of $H_{M}^{\tau}$ or the Poisson bracket $\{,\}_{\circ}$ of $H^{\prime}$ : since the pairing is perfect, we must have $\{,\}_{\star}=\{,\}_{0}$, whence the thesis.

7.5. The case $q \rightarrow 1$ : Specialization of $\mathcal{U}_{\varphi}^{M}(\mathfrak{h})$ to $F^{\infty}\left[G_{M}^{\tau}\right]$. We are going to show that $\mathcal{U}_{\varphi}^{M}(\mathfrak{h})$ is a quantization of $F^{\infty}\left[G_{M}^{\tau}\right]\left(=F^{\infty}\left[G^{\tau}\right]\right)$; such a result can be seen as (Poisson) dual counterpart of $\mathcal{U}_{\varphi}^{M}(\mathfrak{g}) \stackrel{q \rightarrow 1}{\longrightarrow} F\left[H_{M}^{\tau}\right]$ (cf. Theorem 7.4). As usual, we set

$$
\mathcal{U}_{1, \varphi}^{M}(\mathfrak{h}):=\mathcal{U}_{\varphi}^{M}(\mathfrak{h}) /(q-1) \mathcal{U}_{\varphi}^{M}(\mathfrak{h}) \cong \mathcal{U}_{\varphi}^{M}(\mathfrak{h}) \otimes_{k\left[q, q^{-1}\right]} k .
$$


Theorem 7.6. The formal Hopf algebra $\mathcal{U}_{\varphi}^{M}(\mathfrak{h})$ specializes to the formal Poisson Hopf algebra $F^{\infty}\left[G_{M}^{\tau}\right]\left(=F^{\infty}\left[G^{\tau}\right]\right)$ for $q \rightarrow 1$; in other words, there exists an isomorphism of formal Poisson Hopf algebras

$$
\mathcal{U}_{1, \varphi}^{M}(\mathfrak{h}) \cong F^{\infty}\left[G_{M}^{\tau}\right]
$$

Proof. Recall that $F^{\infty}\left[G_{M}^{\tau}\right]=F^{\infty}\left[G^{\tau}\right]$ is isomorphic to the linear dual of $U\left(\mathfrak{g}^{\tau}\right)$, that is $F^{\infty}\left[G_{M}^{\tau}\right] \cong U\left(\mathfrak{g}^{\tau}\right)^{*}$. On the other hand, we have a formal Hopf algebra isomorphism $j_{M}{ }^{-1} \circ \nu_{M}^{\varphi}: U_{q, \varphi}^{M}(\mathfrak{h}) \stackrel{\cong}{\longrightarrow} U_{q, \varphi}^{M^{\prime}}(\mathfrak{g})^{*}$, and Theorem 6.6 ensures that this restricts to

$$
j_{M}{ }^{-1} \circ \nu_{M}^{\varphi}: \mathcal{U}_{\varphi}^{M}(\mathfrak{h}) \stackrel{\cong}{\longrightarrow} \mathfrak{U}_{\varphi}^{M^{\prime}}(\mathfrak{g})^{*} .
$$

When $q \rightarrow 1$, we have that $\mathfrak{U}_{\varphi}^{M^{\prime}}(\mathfrak{g})$ specializes to $U\left(\mathfrak{g}^{\tau}\right)$, therefore (7.1) implies $\mathcal{U}_{1, \varphi}^{M}(\mathfrak{h}) \cong \mathfrak{U}_{\varphi}^{M^{\prime}}(\mathfrak{g})^{*} \otimes_{k\left[q, q^{-1}\right]} k=\mathfrak{U}_{1, \varphi}^{M^{\prime}}(\mathfrak{g})^{*} \cong U\left(\mathfrak{g}^{\tau}\right)^{*}=F^{\infty}\left[G^{\tau}\right]=$ $F^{\infty}\left[G_{M}^{\tau}\right]$, q.e.d.

7.7. The case $q \rightarrow \varepsilon$ : Quantum Frobenius morphisms. Let $\varepsilon$ be a primitive $\ell$-th root of 1 in $k$, for $\ell$ odd $, \ell>d:=\max _{i}\left\{d_{i}\right\}$, and set

$$
\mathfrak{U}_{\varepsilon, \varphi}^{M}(\mathfrak{h}):=\mathfrak{U}_{\varphi}^{M}(\mathfrak{h}) /(q-\varepsilon) \mathfrak{U}_{\varphi}^{M}(\mathfrak{h}) \cong \mathfrak{U}_{\varphi}^{M}(\mathfrak{h}) \otimes_{k\left[q, q^{-1}\right]} k .
$$

First of all we remark that

$$
\mathfrak{U}_{\varepsilon, \varphi}^{M}(\mathfrak{h}) \text { is a usual Hopf algebra over } k \text {, isomorphic to }\left.\mathfrak{H}_{\varphi}^{M}\right|_{q=\varepsilon}
$$

for every element of $\mathfrak{U}_{\varepsilon, \varphi}^{M}(\mathfrak{h})$ is a formal series of terms in $\mathfrak{H}_{\varphi}^{M}$ which is a finite sum modulo $(q-\varepsilon)$, and $\S 5.16$ and Theorem 6.2 tell us that $\Delta\left(\left.\mathfrak{H}_{\varphi}^{M}\right|_{q=\varepsilon}\right) \subseteq$ $\left.\left.\mathfrak{H}_{\varphi}^{M}\right|_{q=\varepsilon} \otimes \mathfrak{H}_{\varphi}^{M}\right|_{q=\varepsilon}$, and $S\left(\left.\mathfrak{H}_{\varphi}^{M}\right|_{q=\varepsilon}\right)=\left.\mathfrak{H}_{\varphi}^{M}\right|_{q=\varepsilon}$. Now we are ready for next result, the analogue for $U_{q, \varphi}^{M}(\mathfrak{h})$ of (3.6).

Theorem 7.8. There exists a Hopf algebras epimorphism

$$
\mathfrak{F}_{\mathfrak{h}} \mathfrak{h}^{\tau}: \mathfrak{U}_{\varepsilon, \varphi}^{M}(\mathfrak{h}) \longrightarrow \mathfrak{U}_{1, \varphi}^{M}(\mathfrak{h}) \cong U\left(\mathfrak{h}^{\tau}\right)
$$

defined (for all $i=1, \ldots, n)$ by

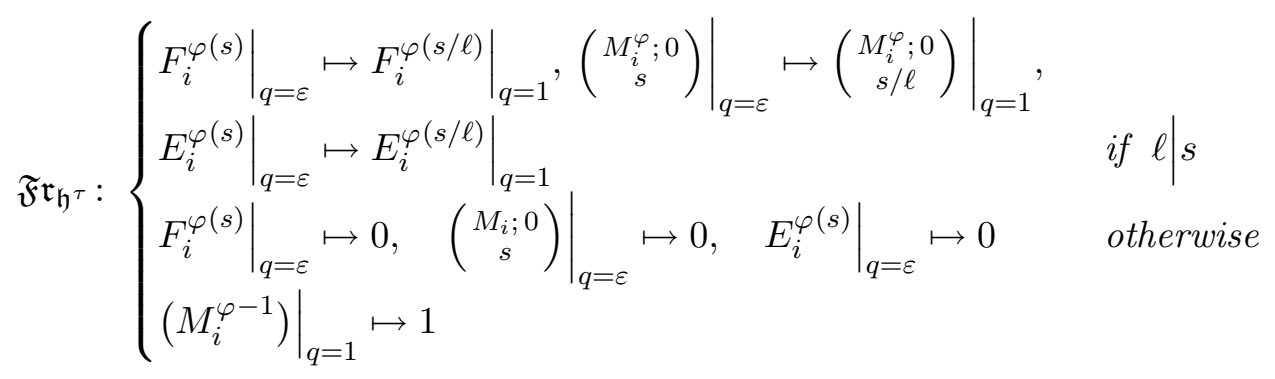


which is adjoint of $\mathcal{F r}_{\mathfrak{g}^{\tau}}$ (cf. (3.9)) with respect to the quantum Poisson pairings, that is

$$
\pi_{1, H_{M^{\prime}}^{\tau}}^{\varphi}\left(\mathfrak{F r}_{\mathfrak{h}^{\tau}}(h), g\right)=\pi_{\varepsilon, H_{M^{\prime}}^{\tau}}^{\varphi}\left(h, \mathcal{F} r_{\mathfrak{g}^{\tau}}(g)\right) \quad \forall h \in \mathfrak{U}_{\varepsilon, \varphi}^{M}(\mathfrak{h}), g \in \mathcal{U}_{1, \varphi}^{M^{\prime}}(\mathfrak{g}) .
$$

Proof. The formulas above uniquely determine an epimorphism $\mathfrak{F}_{\mathfrak{h}}{ }^{\tau}$ - if any - because $\left.\left(F_{i}^{\varphi}\right)^{(s)}\right|_{q=\varepsilon},\left.\left(\begin{array}{c}M_{i}^{\varphi} ; 0 \\ s\end{array}\right)\right|_{q=\varepsilon},\left.\left(M_{i}^{\varphi}\right)^{-1}\right|_{q=\varepsilon},\left.\left(E_{i}^{\varphi}\right)^{(s)}\right|_{q=\varepsilon}$ are (algebraic) generators of $\left.\mathfrak{H}_{\varphi}^{M}\right|_{q=\varepsilon}=\mathfrak{U}_{\varepsilon, \varphi}^{M}(\mathfrak{h})$ (cf. (7.2)). Consider the embedding $\mathcal{F} r_{\mathfrak{g}^{\tau}}: F\left[H_{M^{\prime}}^{\tau}\right] \cong \mathcal{U}_{1, \varphi}^{M^{\prime}}(\mathfrak{g}) \longleftrightarrow \mathcal{U}_{\varepsilon, \varphi}^{M^{\prime}}(\mathfrak{g})$ of Hopf algebras (cf. (3.9)): its linear dual is an epimorphism of formal Hopf algebras $\mathcal{U}_{\varepsilon, \varphi}^{M^{\prime}}(\mathfrak{g})^{*} \longrightarrow \mathcal{U}_{1, \varphi}^{M^{\prime}}(\mathfrak{g})^{*}$. On the other hand we have an embedding $\mathfrak{U}_{\varepsilon, \varphi}^{M}(\mathfrak{h}) \longleftrightarrow \mathcal{U}_{\varepsilon, \varphi}^{M^{\prime}}(\mathfrak{g})^{*}$ provided by the specialized quantum Poisson pairing $\pi_{\varepsilon, H_{M^{\prime}}^{\tau}}^{\varphi}: \mathfrak{U}_{\varepsilon, \varphi}^{M}(\mathfrak{h}) \otimes \mathcal{U}_{\varepsilon, \varphi}^{M^{\prime}}(\mathfrak{g}) \longrightarrow k$.

Now composition yields a morphism $\mathfrak{F r}_{\mathfrak{h}^{\tau}}: \mathfrak{U}_{\varepsilon, \varphi}^{M}(\mathfrak{h}) \longrightarrow \mathcal{U}_{1, \varphi}^{M^{\prime}}(\mathfrak{g})^{*}$; the very construction then gives $\left\langle\mathfrak{F}_{\mathfrak{h}^{\tau}}(h), g\right\rangle=$ $\pi_{1, H_{M^{\prime}}^{\tau}}^{\varphi}\left(\mathfrak{F r}_{\mathfrak{h}^{\tau}}(h), g\right)=\pi_{\varepsilon, H_{M^{\prime}}^{\tau}}^{\varphi}\left(h, \mathcal{F} r_{\mathfrak{g}^{\tau}}(g)\right)$, hence $\mathfrak{F}_{\mathfrak{h}} \mathfrak{h}^{\tau}$ is adjoint of $\mathcal{F} r_{\mathfrak{g}^{\tau}}(g)$, is described by the previous formulas and has image $\mathfrak{U}_{1, \varphi}^{M}(\mathfrak{h})$, q.e.d.

Similar arguments prove next result, which is the analogue of (3.9); as usual, we set

$$
\mathcal{U}_{\varepsilon, \varphi}^{M}(\mathfrak{h}):=\mathcal{U}_{\varphi}^{M}(\mathfrak{h}) /(q-\varepsilon) \mathcal{U}_{\varphi}^{M}(\mathfrak{h}) \cong \mathcal{U}_{\varphi}^{M}(\mathfrak{h}) \otimes_{k\left[q, q^{-1}\right]} k .
$$

Theorem 7.9. (a) There exists a unique continuous monomorphism of formal Hopf algebras

$$
\mathcal{F} r_{\mathfrak{h}^{\tau}}: F^{\infty}\left[G_{M}^{\tau}\right] \cong \mathcal{U}_{1, \varphi}^{M}(\mathfrak{h}) \longleftarrow \mathcal{U}_{\varepsilon, \varphi}^{M}(\mathfrak{h})
$$

defined (for all $\alpha \in R^{+}, \mu \in M$ ) by

$$
\mathcal{F} r_{\mathfrak{h}^{\tau}}:\left.\left.\bar{F}_{\alpha}^{\varphi}\right|_{q=1} \mapsto\left(\bar{F}_{\alpha}^{\varphi}\right)^{\ell}\right|_{q=\varepsilon},\left.\left.\quad L_{\mu}^{\varphi}\right|_{q=1} \mapsto\left(L_{\mu}^{\varphi}\right)^{\ell}\right|_{q=\varepsilon},\left.\left.\quad \bar{E}_{\alpha}^{\varphi}\right|_{q=1} \mapsto\left(\bar{E}_{\alpha}^{\varphi}\right)^{\ell}\right|_{q=\varepsilon}
$$

which is the continuous extension of $\mathfrak{F r}_{G^{\tau}}$ (cf. (4.5)) and is adjoint of $\mathfrak{F r}_{\mathfrak{g}^{\tau}}$ (cf. (3.6)) with respect to quantum Poisson pairings, that is

$$
\pi_{\varepsilon, G_{M}^{\tau}}^{\varphi}\left(\mathcal{F} r_{\mathfrak{h} \tau}(h), g\right)=\pi_{1, G_{M}^{\tau}}^{\varphi}\left(h, \mathfrak{F r}_{\mathfrak{g}^{\tau}}(g)\right) \quad \forall h \in \mathcal{U}_{1, \varphi}^{M}(\mathfrak{h}), g \in \mathfrak{U}_{\varepsilon, \varphi}^{M^{\prime}}(\mathfrak{g}) .
$$

(b) The image $Z_{0}^{\varphi}\left(\cong_{\mathcal{F} r_{\mathfrak{h}} \tau} \mathcal{U}_{1, \varphi}^{M}(\mathfrak{h})\right)$ of $\mathcal{F} r_{\mathfrak{h}^{\tau}}$ is a formal Hopf subalgebra contained in the centre of $\mathcal{U}_{\varepsilon, \varphi}^{M}(\mathfrak{h})$.

(c) The set $\left\{\mathcal{F}_{\ell \phi} \cdot B_{\ell \phi, \ell \tau, \ell \eta}^{\varphi} \cdot \mathcal{E}_{\ell \eta} \mid \phi \in \mathbb{N}^{N}, \tau \in \mathbb{N}^{n}, \eta \in \mathbb{N}^{N}\right\}$ is a pseudobasis of $Z_{0}^{\varphi}$ over $k$. 
(d) The set $\left\{\mathcal{F}_{\phi} \cdot B_{\phi, \tau, \eta}^{\varphi} \cdot \mathcal{E}_{\eta} \mid \tau \in\{0,1, \ldots, \ell-1\}^{n} ; \phi, \eta \in\{0,1, \ldots, \ell-1\}^{N}\right\}$ is a basis of $\mathcal{U}_{\varepsilon, \varphi}^{M}(\mathfrak{h})$ over $Z_{0}$; therefore also the set of ordered PBW monomials $\left\{\mathcal{F}_{\phi} \cdot M_{\mu}^{\varphi} \cdot \mathcal{E}_{\eta} \mid \mu \in\{0,1, \ldots, \ell-1\}^{n} ; \phi, \eta \in\{0,1, \ldots, \ell-1\}^{N}\right\}$ is a basis of $\mathcal{U}_{\varepsilon, \varphi}^{M}(\mathfrak{h})$ over $Z_{0}^{\varphi}$. Thus $\mathcal{U}_{\varepsilon, \varphi}^{M}(\mathfrak{h})$ is a free module of rank $\ell^{\operatorname{dim}\left(H^{\tau}\right)}$ over $Z_{0}^{\varphi}$.

Proof. (a) Since $\left.\bar{F}_{\alpha}^{\varphi}\right|_{q=1},\left.L_{\mu}^{\varphi}\right|_{q=1},\left.\bar{E}_{\alpha}^{\varphi}\right|_{q=1}\left(\alpha \in R^{+}, \mu \in M\right)$ are topological generators of $\mathcal{U}_{1, \varphi}^{M}(\mathfrak{h})$, the formulas above uniquely determine a continuous monomorphism $\mathcal{F} r_{\mathfrak{h}^{\tau}}$, if any. Now consider $\mathfrak{F r}_{\mathfrak{g}^{\tau}}: \mathfrak{U}_{\varepsilon, \varphi}^{M^{\prime}}(\mathfrak{g}) \longrightarrow \mathfrak{U}_{1, \varphi}^{M^{\prime}}(\mathfrak{g}) \cong$ $U\left(\mathfrak{g}^{\tau}\right)$ (cf. (3.6)), a Hopf epimorphism, and its dual, a formal Hopf monomorphism $\mathfrak{U}_{1, \varphi}^{M^{\prime}}(\mathfrak{g})^{*} \longleftrightarrow \mathfrak{U}_{\varepsilon, \varphi}^{M^{\prime}}(\mathfrak{g})^{*}$; composing the latter with the isomorphisms $\mathcal{U}_{1, \varphi}^{M}(\mathfrak{h}) \stackrel{\cong}{\longrightarrow} \mathfrak{U}_{1, \varphi}^{M^{\prime}}(\mathfrak{g})^{*}, \mathfrak{U}_{\varepsilon, \varphi}^{M^{\prime}}(\mathfrak{g})^{*} \stackrel{\cong}{\longrightarrow} \mathcal{U}_{\varepsilon, \varphi}^{M}(\mathfrak{h})$ (given by specialized quantum Poisson pairings) provides a monomorphism $\mathcal{F} r_{\mathfrak{h}^{\tau}}: \mathcal{U}_{1, \varphi}^{M}(\mathfrak{h}) \longleftrightarrow \mathcal{U}_{\varepsilon, \varphi}^{M}(\mathfrak{h})$; then

$$
\begin{aligned}
&\left\langle\mathcal{F} r_{\mathfrak{h}^{\tau}}(h), g\right\rangle=\pi_{\varepsilon, G_{M}^{\tau}}^{\varphi}\left(\mathcal{F} r_{\mathfrak{h}^{\tau}}(h), g\right)=\pi_{1, G_{M}^{\tau}}^{\varphi}\left(h, \mathfrak{F r}_{\mathfrak{g}^{\tau}}(g)\right) \\
& \forall h \in \mathcal{U}_{1, \varphi}^{M}(\mathfrak{h}), x \in \mathfrak{U}_{\varepsilon}^{M^{\prime}}(\mathfrak{g})
\end{aligned}
$$

hence $\mathcal{F} r_{\mathfrak{h}}$ is described by the formulas above. Moreover, with notation of $\S 6.1$ and $\S 6.3$,

$$
\begin{aligned}
\left\langle\mathcal{F} r_{\mathfrak{h}^{\tau}}\left(Y_{\phi, \zeta, \eta}^{\varphi}\right), X_{\epsilon, \theta, \psi}\right\rangle & =\pi_{\varepsilon, G_{M}^{\tau}}^{\varphi}\left(\mathcal{F} r_{\mathfrak{h}^{\tau}}\left(Y_{\phi, \zeta, \eta}^{\varphi}\right), X_{\epsilon, \theta, \psi}\right) \\
& =\pi_{1, G_{M}^{\tau}}^{\varphi}\left(Y_{\phi, \zeta, \eta}^{\varphi}, \mathfrak{F r}_{\mathfrak{g}^{\tau}}\left(X_{\epsilon, \theta, \psi}\right)\right) \\
& =\chi_{\ell \mathbb{N}^{N}}(\epsilon) \cdot \chi_{\ell \mathbb{N}^{n}}(\theta) \cdot \chi_{\ell \mathbb{N}^{N}}(\psi) \cdot\left\langle Y_{\phi, \zeta, \eta}^{\varphi}, X_{\frac{1}{\ell} \cdot \epsilon, \frac{1}{\ell} \cdot \theta, \frac{1}{\ell} \cdot \psi}\right\rangle \\
& =\chi_{\ell \cdot\left(\mathbb{N}^{N} \times \mathbb{N}^{n} \times \mathbb{N}^{N}\right)}(\epsilon, \theta, \psi) \cdot \delta_{\ell(\phi, \zeta, \eta),(\epsilon, \theta, \psi)}
\end{aligned}
$$

(where $\chi_{\ell S}$ is the characteristic function of the sublattice $\ell S \subseteq S$, for any abelian semigroup $S$ ), hence $\mathcal{F} r_{\mathfrak{h}^{\tau}}\left(Y_{\phi, \zeta, \eta}^{\varphi}\right)=Y_{\ell \phi, \ell \zeta, \ell \eta}^{\varphi}$ for all $\phi, \zeta, \eta$, thus $\mathcal{F} r_{\mathfrak{h}^{\tau}}$ maps elements of the pseudobasis $(6.2)$ of $\mathcal{U}_{1, \varphi}^{M}(\mathfrak{h})$ onto elements of the analogous pseudobasis of $\mathcal{U}_{\varepsilon, \varphi}^{M}(\mathfrak{h})$ : Therefore $\mathcal{F} r_{\mathfrak{h}^{\tau}}$ is continuous.

Finally, since $\mathfrak{F r}_{G^{\tau}}: F\left[G_{M}^{\tau}\right] \cong \mathfrak{F}_{1, \varphi}^{M}[G] \hookrightarrow \mathfrak{F}_{\varepsilon, \varphi}^{M}[G]$ too is defined as (Hopf) dual of $\mathfrak{F r}_{\mathfrak{g}^{\tau}}: \mathfrak{U}_{\varepsilon, \varphi}^{M^{\prime}}(\mathfrak{g}) \rightarrow \mathfrak{U}_{1, \varphi}^{M^{\prime}}(\mathfrak{g}) \cong U\left(\mathfrak{g}^{\tau}\right)$ (cf. [DL], Proposition 6.4), then $\mathcal{F} r_{\mathfrak{h}^{\tau}}: F^{\infty}\left[G_{M}^{\tau}\right] \cong \mathcal{U}_{1, \varphi}^{M}(\mathfrak{h}) \hookrightarrow \mathcal{U}_{\varepsilon, \varphi}^{M}(\mathfrak{h})$ is extension of $\mathfrak{F r}_{G^{\tau}}: F\left[G_{M}^{\tau}\right] \cong$ $\mathfrak{F}_{1, \varphi}^{M}[G] \hookrightarrow \mathfrak{F}_{\varepsilon, \varphi}^{M}[G]$; since $\mathfrak{F}_{\varphi}^{M}[G]$ is dense in $\mathfrak{U}_{\varphi}^{M^{\prime}}(\mathfrak{g})^{*} \cong \mathcal{U}_{\varphi}^{M}(\mathfrak{h})$ it is clear that this extension is by continuity.

(b) This easily follows from the analogous result for $\mathcal{U}_{\varepsilon, \varphi}^{M}(\mathfrak{g})$ (cf. [DP], Theorem 19.1) and comparison among $\mathcal{U}_{\varepsilon, \varphi}^{M}(\mathfrak{g})$ and $\mathcal{U}_{\varepsilon, \varphi}^{M}(\mathfrak{h})$.

(c) This follows from the previous analysis, namely from $\mathcal{F} r_{\mathfrak{h}^{\tau}}\left(Y_{\phi, \zeta, \eta}^{\varphi}\right)=$ $Y_{\ell \phi, \ell \zeta, \ell \eta}^{\varphi}$. 
(d) The span of $\left\{B_{\phi, \zeta, \eta}^{\varphi} \mid(\phi, \zeta, \eta) \in \ell\left(\mathbb{N}^{N} \times \mathbb{N}^{n} \times \mathbb{N}^{N}\right)\right\}$ (inside $\left.\mathcal{U}_{\varepsilon, \varphi}^{M}(\mathfrak{h})\right)$ coincides with the span of $\left\{L_{\mu}^{\varphi} \mid \lambda \in \ell \mathbb{N}^{n}=\ell M_{+}\right\}$; from this and from the explicit form of the pseudobasis of $\mathcal{U}_{\varphi}^{M}(\mathfrak{h})$ we get the claim.

At last we prove the dual counterpart of (4.5), regarding

$$
\mathcal{F}_{\varepsilon, \varphi}^{M}[G]:=\mathcal{F}_{\varphi}^{M}[G] /(q-\varepsilon) \mathcal{F}_{\varphi}^{M}[G] \cong \mathcal{F}_{\varphi}^{M}[G] \otimes_{k\left[q, q^{-1}\right]} k
$$

note that we obtain a quantum Frobenius morphism which is surjective instead of injective.

Theorem 7.10. There exists a Hopf algebra epimorphism

$$
\mathcal{F} r_{H^{\tau}}: \mathcal{F}_{\varepsilon, \varphi}^{M}[G] \longrightarrow \mathcal{F}_{1, \varphi}^{M}[G] \cong U\left(\mathfrak{h}^{\tau}\right)
$$

dual of $\mathcal{F} r_{\mathfrak{g}^{\tau}}: F\left[H_{M^{\prime}}^{\tau}\right] \cong \mathcal{U}_{1, \varphi}^{M^{\prime}}(\mathfrak{g}) \longleftrightarrow \mathcal{U}_{\varepsilon, \varphi}^{M^{\prime}}(\mathfrak{g})$ and adjoint of it with respect to the quantum Poisson pairings.

Proof. Since $\mathcal{F}_{\varepsilon, \varphi}^{M}[G] \longleftrightarrow \mathfrak{U}_{\varepsilon, \varphi}^{M}(\mathfrak{h})$, we can restrict $\mathfrak{F}_{\mathfrak{h}} \tau$ to $\mathcal{F}_{\varepsilon, \varphi}^{M}[G]$, thus obtaining a Hopf algebra morphism $\mathcal{F} r_{H^{\tau}}: \mathcal{F}_{\varepsilon, \varphi}^{M}[G] \longrightarrow \mathfrak{U}_{1, \varphi}^{M}(\mathfrak{h}) \cong U\left(\mathfrak{h}^{\tau}\right)$. But Theorem 9.3 gives $\mathcal{F}_{1, \varphi}^{M}[G]=\mathfrak{U}_{1, \varphi}^{M}(\mathfrak{h}) \cong U\left(\mathfrak{h}^{\tau}\right)$, whence the thesis.

We call also $\mathfrak{F}_{\mathfrak{h}^{\tau}}, \mathcal{F} r_{\mathfrak{h}^{\tau}}$, and $\mathcal{F} r_{H^{\tau}}$ quantum Frobenius morphisms, because they can be thought of as liftings of classical Frobenius morphisms to characteristic zero.

7.11. Specializations of quantum Poisson pairings. From $\S \S 7.2-6$ we get that the Hopf pairings $\pi_{q, H_{M}^{\tau}}^{\varphi}: \mathfrak{U}_{\varphi}^{M^{\prime}}(\mathfrak{h}) \otimes \mathcal{U}_{\varphi}^{M}(\mathfrak{g}) \longrightarrow k\left[q, q^{-1}\right], \pi_{q, G_{M}^{\tau}}^{\varphi}:$ $\mathcal{U}_{\varphi}^{M}(\mathfrak{h}) \otimes \mathfrak{U}_{\varphi}^{M^{\prime}}(\mathfrak{g}) \longrightarrow k\left[q, q^{-1}\right]$ (cf. 6.12) respectively specialize to the natural Hopf pairings $\pi_{H_{M}^{\tau}}: U\left(\mathfrak{h}^{\tau}\right) \otimes F\left[H_{M}^{\tau}\right] \longrightarrow k, \pi_{G_{M}^{\tau}}: F^{\infty}\left[G_{M}^{\tau}\right] \otimes U\left(\mathfrak{g}^{\tau}\right) \longrightarrow k$; in other words, $\left.\pi_{q, H_{M}^{\tau}}^{\varphi}(\hat{h}, \tilde{g})\right|_{q=1}=\pi_{H_{M}^{\tau}}\left(\left.\hat{h}\right|_{q=1},\left.\tilde{g}\right|_{q=1}\right),\left.\pi_{q, G_{M}^{\tau}}^{\varphi}(\tilde{h}, \hat{g})\right|_{q=1}=$ $\pi_{G_{M}^{\tau}}\left(\left.\tilde{h}\right|_{q=1},\left.\hat{g}\right|_{q=1}\right)$. Thus the quantum Poisson pairing is a quantization of the classical Hopf pairing on both our Poisson groups dual of each other. In addition we show that it can also be thought of as a quantization of the classical Poisson pairing $\pi_{\mathcal{P}}^{\tau}: \mathfrak{h}^{\tau} \otimes \mathfrak{g}^{\tau} \rightarrow k$, and of new pairings between function algebras. We use notations $[]:,=m-m^{o p}, \nabla:=\Delta-\Delta^{o p}$ (superscript "op" denoting opposite operation). by

First of all, we define a suitable grading on $\mathfrak{U}_{\varphi}^{Q}(\mathfrak{g})\left(\right.$ as a $k\left[q, q^{-1}\right]$-module)

$$
\begin{aligned}
& \operatorname{deg}\left(\prod_{r=N}^{1}\left(E_{\alpha^{r}}^{\varphi}\right)^{\left(m_{r}\right)} \cdot \prod_{i=1}^{n}\left(\begin{array}{c}
K_{i}^{\varphi} ; 0 \\
t_{i}
\end{array}\right)\left(K_{i}^{\varphi}\right)^{-E n t\left(t_{i} / 2\right)} \cdot \prod_{r=1}^{N}\left(F_{\alpha^{r}}^{\varphi}\right)^{\left(n_{r}\right)}\right):= \\
& :=\sum_{r=N}^{1} m_{r}+\sum_{i=1}^{n} t_{i}+\sum_{r=1}^{N} n_{r}
\end{aligned}
$$


and linear extension. Then let $k\left[q, q^{-1}\right]=: \mathfrak{U}_{0}^{\varphi} \subset \mathfrak{U}_{1}^{\varphi} \subset \cdots \subset \mathfrak{U}_{h}^{\varphi} \subset \cdots(\subset$ $\left.\mathfrak{U}_{\varphi}^{Q}(\mathfrak{g})\right)$ be the associated filtration, and set $\partial(x):=h$ for all $x \in \mathfrak{U}_{h}^{\varphi} \backslash \mathfrak{U}_{h-1}^{\varphi}$. Notice that a similar notion of degree exists for $U\left(\mathfrak{g}^{\tau}\right)$, defined by means of the filtration $U_{0} \subset U_{1} \subset \cdots \subset U_{N} \subset \cdots \subset U\left(\mathfrak{g}^{\tau}\right)$ induced by the canonical filtration of $T\left(\mathfrak{g}^{\tau}\right)$ (the tensor algebra on $\mathfrak{g}^{\tau}$ ), and similarly for $U\left(\mathfrak{g}^{\tau}\right) \otimes U\left(\mathfrak{g}^{\tau}\right)$. Finally define

$$
\pi_{q, \mathcal{P}}^{\varphi}(h, g):=(q-1)^{\partial(g)} \cdot \pi_{q}^{\varphi}(h, g) \quad \forall h \in \mathfrak{U}_{\varphi}^{Q}(\mathfrak{h}), g \in \mathfrak{U}_{\varphi}^{Q}(\mathfrak{g}) ;
$$

this yields a perfect pairing $\pi_{q, \mathcal{P}}: \mathfrak{U}_{\varphi}^{Q}(\mathfrak{h}) \times \mathfrak{U}_{\varphi}^{Q}(\mathfrak{g}) \longrightarrow k\left[q, q^{-1}\right]_{(q-1)}$ (the latter being the localized ring). In particular $\pi_{q, \mathcal{P}}^{\varphi}$ can be specialized at $q=1$.

Theorem 7.12. $\pi_{q, \mathcal{P}}^{\varphi}: \mathfrak{U}_{\varphi}^{Q}(\mathfrak{h}) \times \mathfrak{U}_{\varphi}^{Q}(\mathfrak{g}) \rightarrow k\left[q, q^{-1}\right]_{(q-1)}$ specializes to a pairing

$$
\pi_{\mathcal{P}}^{\tau}: U\left(\mathfrak{h}^{\tau}\right) \times U\left(\mathfrak{g}^{\tau}\right) \longrightarrow k
$$

which extends the Lie bialgebra pairing $\pi_{\mathcal{P}}^{\tau}: \mathfrak{h}^{\tau} \otimes \mathfrak{g}^{\tau} \longrightarrow k($ cf. 11.2$)$ and is such that

$$
\begin{gathered}
\pi_{\mathcal{P}}(\alpha \cdot x+\beta \cdot y, z)=\alpha \cdot \pi_{\mathcal{P}}(x, z)+\beta \cdot \pi_{\mathcal{P}}(y, z) \\
\pi_{\mathcal{P}}(x, \alpha \cdot u+\beta \cdot v)=\alpha \cdot \pi_{\mathcal{P}}(x, u)+\beta \cdot \pi_{\mathcal{P}}(x, v) \\
\pi_{\mathcal{P}}(x \cdot y, z)=\pi_{\mathcal{P}}(x \otimes y, \Delta(z)), \quad \pi_{\mathcal{P}}(x, z \cdot w)=\pi_{\mathcal{P}}(\Delta(x), z \otimes w) \\
\pi_{\mathcal{P}}([x, y], z)=\pi_{\mathcal{P}}(x \otimes y, \delta(z)), \quad \pi_{\mathcal{P}}(x,[z, w])=\pi_{\mathcal{P}}(\delta(x), z \otimes w)
\end{gathered}
$$

for all $\alpha, \beta \in k, x, y \in U(\mathfrak{h}), z, w, u, v \in U(\mathfrak{g})$ such that $\partial(\alpha \cdot u+\beta \cdot v)=$ $\partial(u)=\partial(v)$.

Proof. Let $x \in U\left(\mathfrak{h}^{\tau}\right), z \in U\left(\mathfrak{g}^{\tau}\right)$, and pick $x^{\prime} \in \mathfrak{U}_{\varphi}^{Q}(\mathfrak{h}), z^{\prime} \in \mathfrak{U}_{\varphi}^{Q}(\mathfrak{g})$, such that $x=\left.x^{\prime}\right|_{q=1}, z=\left.z^{\prime}\right|_{q=1}$. By definition, $\pi_{\mathcal{P}}(x, z)$ is given by

$$
\pi_{\mathcal{P}}(x, z):=\left.\pi_{q, \mathcal{P}}\left(x^{\prime}, z^{\prime}\right)\right|_{q=1}=\left.\left((q-1)^{\partial\left(z^{\prime}\right)} \cdot \pi_{q}\left(x^{\prime}, z^{\prime}\right)\right)\right|_{q=1}
$$

in particular, we can select $x^{\prime}$ and $z^{\prime}$ such that $\partial\left(x^{\prime}\right)=\partial(x), \partial\left(z^{\prime}\right)=\partial(z)$. Now, the first two identities follows directly from similar properties for $\pi_{q, \mathcal{P}}$, which are directly implied by definitions. As for the other identities, using Leibnitz' and co-Leibnitz' rules and identities $\partial(x \cdot y)=\partial(x)+\partial(y)=$ $\partial(x \otimes y)$ we are easily reduced to prove that they hold for $x, y \in \mathfrak{h}$ and $z, w \in \mathfrak{g}$, which again follows from definition. Finally to prove that $\pi_{\mathcal{P}}$ is an extension of the classical Poisson pairing a straightforward check works. 
7.13. The pairings $F\left[G^{\tau}\right] \times F\left[H^{\tau}\right] \longrightarrow k, F^{\infty}\left[G^{\tau}\right] \times F\left[H^{\tau}\right] \longrightarrow k$. The construction in $\S 7.11$ can be reversed as follows. Define a grading on $\mathcal{U}_{\varphi}^{P}(\mathfrak{g})$ (as a $k\left[q, q^{-1}\right]$-module) by

$$
\begin{aligned}
& \operatorname{deg}\left(\prod_{r=N}^{1}\left(\bar{E}_{\alpha^{r}}^{\varphi}\right)^{m_{r}} \cdot \prod_{i=1}^{n}\left(\left(L_{i}^{\varphi}\right)^{ \pm 1}-1\right)^{l_{i}} \cdot \prod_{r=1}^{N}\left(\bar{F}_{\alpha^{r}}^{\varphi}\right)^{n_{r}}\right):= \\
& :=\sum_{r=N}^{1} m_{r}+\sum_{i=1}^{n} l_{i}+\sum_{r=1}^{N} n_{r}
\end{aligned}
$$

and linear extension; then let $k\left[q, q^{-1}\right]=: \mathcal{U}_{0}^{\varphi} \subset \mathcal{U}_{1}^{\varphi} \subset \cdots \subset \mathcal{U}_{h}^{\varphi} \subset \cdots(\subset$ $\left.\mathcal{U}_{\varphi}^{P}(\mathfrak{g})\right)$ be the associated filtration, and set $\partial(x):=h$ for all $x \in \mathcal{U}_{h}^{\varphi} \backslash \mathcal{U}_{h-1}^{\varphi}$ $(h \in \mathbb{N})$. Then extend $\pi_{q}^{\varphi}: U_{q, \varphi}^{P}(\mathfrak{h}) \otimes U_{q, \varphi}^{Q}(\mathfrak{g}) \longrightarrow k(q)$ to a perfect pairing of formal Hopf algebras $\pi_{q}^{\varphi}: U_{q, \varphi}^{P}(\mathfrak{h}) \otimes U_{q, \varphi}^{P}(\mathfrak{g}) \longrightarrow k\left(q^{1 / D}, q^{-1 / D}\right)$ (where $D$ is the determinant of the Cartan matrix) by the rule $\pi_{q}^{\varphi}\left(L_{\lambda}, L_{\mu}\right):=q^{(\lambda \mid \mu)}$ (where $(\lambda \mid \mu)$ is defined in $\S 1.1$ ). Finally define

$$
\pi_{q, \varphi}^{\mathcal{P}}(h, g):=(q-1)^{-\partial(g)} \cdot \pi_{q}^{\varphi}(h, g) \quad \forall h \in \mathcal{U}_{\varphi}^{P}(\mathfrak{h}), g \in \mathcal{U}_{\varphi}^{P}(\mathfrak{g}) ;
$$

this yields a perfect pairing $\pi_{q, \varphi}^{\mathcal{P}}: \mathcal{U}_{\varphi}^{P}(\mathfrak{h}) \times \mathcal{U}_{\varphi}^{P}(\mathfrak{g}) \longrightarrow k\left[q^{1 / d}, q^{-1 / d}\right]$, whose set of values is an ideal coprime with the principal ideal $\left(q^{1 / D}-1\right)$; furthermore, restriction gives also a similar pairing $\pi_{q, \varphi}^{\mathcal{P}}: \mathfrak{F}_{\varphi}^{P}[G] \times \mathcal{U}_{\varphi}^{P}(\mathfrak{g}) \longrightarrow$ $k\left[q^{1 / d}, q^{-1 / d}\right]$.

Now we can specialize these pairings at $q=q^{1 / d}=1$, which gives the following:

Teorema 7.14. The pairing $\pi_{q, \varphi}^{\mathcal{P}}: \mathcal{U}_{\varphi}^{P}(\mathfrak{h}) \times \mathcal{U}_{\varphi}^{P}(\mathfrak{g}) \longrightarrow k\left[q^{1 / d}, q^{-1 / d}\right]$ and the pairing $\pi_{q, \varphi}^{\mathcal{P}}: \mathfrak{F}_{\varphi}^{P}[G] \times \mathcal{U}^{P}(\mathfrak{g}) \longrightarrow k\left[q^{1 / d}, q^{-1 / d}\right]$ specialize to pairings

$$
\pi_{\tau}^{\mathcal{P}}: F^{\infty}\left[G^{\tau}\right] \otimes F\left[H^{\tau}\right] \longrightarrow k, \quad \pi_{\tau}^{\mathcal{P}}: F\left[G^{\tau}\right] \otimes F\left[H^{\tau}\right] \longrightarrow k
$$

such that

$$
\begin{gathered}
\pi_{\tau}^{\mathcal{P}}(\alpha \cdot x+\beta \cdot y, z)=\alpha \cdot \pi_{\tau}^{\mathcal{P}}(x, z)+\beta \cdot \pi_{\tau}^{\mathcal{P}}(y, z) \\
\pi_{\tau}^{\mathcal{P}}(x, \alpha \cdot u+\beta \cdot v)=\alpha \cdot \pi_{\tau}^{\mathcal{P}}(x, u)+\beta \cdot \pi_{\tau}^{\mathcal{P}}(x, v) \\
\pi_{\tau}^{\mathcal{P}}(x \cdot y, z)=\pi_{\tau}^{\mathcal{P}}(x \otimes y, \Delta(z)), \quad \pi_{\tau}^{\mathcal{P}}(x, z \cdot w)=\pi_{\tau}^{\mathcal{P}}(\Delta(x), z \otimes w) \\
\pi_{\tau}^{\mathcal{P}}(\{x, y\}, z)=\pi_{\tau}^{\mathcal{P}}(x \otimes y, \nabla(z)), \quad \pi_{\tau}^{\mathcal{P}}(x,\{z, w\})=\pi_{\tau}^{\mathcal{P}}(\nabla(x), z \otimes w)
\end{gathered}
$$

for all $\alpha, \beta \in k, x, y \in F\left[G^{\tau}\right]$ or $x, y \in F^{\infty}\left[G^{\tau}\right], z, u, v \in F\left[H^{\tau}\right]$ such that $\partial(\alpha \cdot u+\beta \cdot v)=\partial(u)=\partial(v)\left(\right.$ with $\partial(x):=\partial\left(x^{\prime}\right)$ for any $x^{\prime} \in \mathcal{U}_{\varphi}^{P}(\mathfrak{g})$ such that $\left.\left.x^{\prime}\right|_{q=1}=x\right)$.

Proof. Just mimick the proof of Theorem 7.12 above. 


\section{Formal quantum groups.}

8.1. Formal quantum groups versus quantum formal groups. The heading of this subsection is not a play on words: in fact we wish to discuss the possibility to develop two different notions which are to be quantum analog of the notion of formal group; the different position of the word quantum in the previous expressions just refer to two different ways to conceive the notion of formal group, which give rise to two different "quantizations".

In $\S 7.1$ we start from the fact that a formal group is given by a commutative formal Hopf algebra, which can be realized as $U(\mathfrak{g})^{*}$ — the dual of $U(\mathfrak{g})$ - thus we defined the quantum formal groups as spectra of formal Hopf algebras, and we looked at $U_{q, \varphi}^{M}(\mathfrak{g})^{*}$.

An alternative method stems from the fact that the topological Hopf algebra of a formal group may be obtained as a suitable completion of a usual Hopf algebra. Namely, let $F^{\infty}[G]$ be the formal Hopf algebra of a given formal group; let $G$ be an algebraic group with associated formal group equal to the given one; let $\mathfrak{m}_{e}$ be the maximal ideal of $F[G]$ associated to the identity $e \in G$; then $F^{\infty}[G]$ is the $\mathfrak{m}_{e}$-adic completion of $F[G]$. Moreover we remark that $\mathfrak{m}_{e}=\mathfrak{e}:=\operatorname{Ker}(\epsilon)$, where $\epsilon$ is the counit of $F[G]$.

The previous remarks motivate the following way of "quantizing" $F^{\infty}[G]$ : first, constructing a Hopf algebra $F_{q}[G]$ which quantizes $F[G]$; second, constructing the $\mathfrak{E}$-adic completion of $F_{q}[G]$, with $\mathfrak{E}:=\operatorname{Ker}\left(\epsilon: F_{q}[G] \rightarrow k(q)\right)$. We shall call an object obtained in this way formal quantum group. When considering formal Poisson groups we require also that such a quantization is one of the Poisson structure.

We have all the ingredients to perform this construction. The first steps are trivial.

Definition 8.2. Let $M$ be a lattice as in $\S 2.2$, and let $F_{q, \varphi}^{M}[G], \mathfrak{F}_{\varphi}^{M}[G]$, and $\mathcal{F}_{\varphi}^{M}[G]$ be the quantum function algebras defined in $\S 4$.

Let $\mathbf{E}_{\varphi}:=\operatorname{Ker}\left(\epsilon: F_{q, \varphi}^{M}[G] \longrightarrow k(q)\right), \mathfrak{E}_{\varphi}:=\operatorname{Ker}\left(\epsilon: \mathfrak{F}_{\varphi}^{M}[G]\right.$ $\left.k\left[q, q^{-1}\right]\right)$, and $\mathcal{E}_{\varphi}:=\operatorname{Ker}\left(\epsilon: \mathcal{F}_{\varphi}^{M}[G] \longrightarrow k\left[q, q^{-1}\right]\right)$. Then we define

$$
\begin{aligned}
F_{q, \varphi}^{M, \infty}[G]:=\mathbf{E}_{\varphi} \text {-adic completion of } F_{q, \varphi}^{M}[G] \\
\mathfrak{F}_{\varphi}^{M, \infty}[G]:=\mathfrak{E}_{\varphi} \text {-adic completion of } \mathfrak{F}_{\varphi}^{M}[G] \\
\mathcal{F}_{\varphi}^{M, \infty}[G]:=(q-1) \cdot \mathcal{E}_{\varphi} \text {-adic completion of } \mathcal{F}_{\varphi}^{M}[G]
\end{aligned}
$$

Lemma 8.3. Let $H$ be a Hopf algebra over a ring $R$, let $\mathbb{E}$ be the kernel of the counit of $H$, and let $u \in R$ be a non-invertible element of $R$.

(a) Let $\widehat{H}$ be the $\mathbb{E}$-adic completion of $H$. There exists a unique structure of topological Hopf algebra over $R$ on $\widehat{H}$ which extends by continuity that of $H$. 
(b) Let $\widehat{H}_{u}$ be the $u \cdot \mathbb{E}$-adic completion of $H$. There exists a unique structure of topological Hopf algebra over $R$ on $\widehat{H}_{u}$ which extends by continuity that of $H$.

Proposition 8.4. $\quad \mathfrak{F}_{\varphi}^{M, \infty}[G]$ and $\mathcal{F}_{\varphi}^{M, \infty}[G]$ are $k\left[q, q^{-1}\right]$-integer forms of $F_{q, \varphi}^{M, \infty}[G]$ as topological Hopf algebras.

Definition 8.5. Let $\epsilon^{\prime}: \mathbf{H}_{\varphi}^{M} \longrightarrow k(q)$ be the $k(q)$-algebra morphism defined by $\epsilon^{\prime}\left(F_{i}^{\varphi}\right):=0, \epsilon^{\prime}\left(L_{\mu}^{\varphi}\right):=1, \epsilon^{\prime}\left(E_{i}^{\varphi}\right):=0,(\forall i=1, \ldots, n, \mu \in M)$ and set $\mathbb{E}_{\varphi}^{\prime}:=\operatorname{Ker}\left(\epsilon^{\prime}\right), \widetilde{\mathbb{E}}_{\varphi}^{\prime}:=\mathbb{E}_{\varphi}^{\prime} \cap \mathcal{H}_{\varphi}^{M}, \widehat{\mathbb{E}}_{\varphi}^{\prime}:=\mathbb{E}_{\varphi}^{\prime} \cap \mathfrak{H}_{\varphi}^{M}$. We call $U_{q, \varphi}^{M, \infty}(\mathfrak{h})$ the $\mathbb{E}_{\varphi}^{\prime}$-adic completion of $\mathbf{H}_{\varphi}^{M}, \mathcal{U}_{\varphi}^{M, \infty}(\mathfrak{h})$ the $\widetilde{\mathbb{E}}_{\varphi}^{\prime}$-adic completion of $\mathcal{H}_{\varphi}^{M}$, and $\mathfrak{U}_{\varphi}^{M, \infty}(\mathfrak{h})$ the $(q-1) \cdot \widehat{\mathbb{E}}_{\varphi}^{\prime}$-adic completion of $\mathfrak{H}_{\varphi}^{M}$, with its natural structure of topological $k\left[q, q^{-1}\right]$-algebra.

Proposition 8.6. There exists a unique isomorphism of topological $k(q)$ algebras $^{5} \xi_{M}^{\varphi, \infty}: F_{q, \varphi}^{M, \infty}[G] \cong U_{q, \varphi}^{M, \infty}(\mathfrak{h})$ which extends $\xi_{M}^{\varphi}: F_{q, \varphi}^{M}[G] \longleftrightarrow$ $\mathbf{H}_{\varphi}^{M}$ and $\xi_{M}^{\varphi}: F_{q, \varphi}^{M}[G]\left[\psi_{-\rho}^{-1}\right] \stackrel{\cong}{\longrightarrow} \mathbf{H}_{\varphi}^{M}$. It restricts to $\mathfrak{F}_{\varphi}^{M, \infty}[G] \stackrel{\cong}{\longrightarrow} \mathcal{U}_{\varphi}^{M, \infty}(\mathfrak{h})$ (which extends $\xi_{M}^{\varphi}: \mathfrak{F}_{\varphi}^{M}[G] \longleftrightarrow \mathcal{A}_{\varphi}^{M}$ and $\xi_{M}^{\varphi}: \mathfrak{F}_{\varphi}^{M}[G]\left[\psi_{-\rho}^{-1}\right] \stackrel{\cong}{\longrightarrow} \mathcal{A}_{\varphi}^{M}$ ) and to $\mathcal{F}_{\varphi}^{M, \infty}[G] \stackrel{\cong}{\longrightarrow} \mathfrak{U}_{\varphi}^{M, \infty}(\mathfrak{h})$ (which extends $\xi_{M}^{\varphi}: \mathcal{F}_{\varphi}^{M}[G] \longleftrightarrow \mathfrak{A}_{\varphi}^{M}$ and $\xi_{M}^{\varphi}$ : $\left.\mathcal{F}_{\varphi}^{M}[G]\left[\psi_{-\rho}^{-1}\right] \stackrel{\cong}{\cong} \mathfrak{A}_{\varphi}^{M}\right)$. Then by push-out the right-hand side algebras get structures of topogical Hopf algebras, so that $\xi_{M}^{\varphi, \infty}$ is always an isomorphism of topological Hopf algebras.

Proof. From definitions, Theorem 6.11, and formulas for $\epsilon: U_{q, \varphi}^{M}(\mathfrak{h}) \rightarrow k(q)$ in $\S 6.9$ it follows that $\xi_{M}^{\varphi}\left(\mathbf{E}_{\varphi}\right) \subseteq \mathbb{E}_{\varphi}^{\prime}$, hence there exists a unique continuous extension of $\xi_{M}^{\varphi}, \xi_{M}^{\varphi, \infty}: F_{q, \varphi}^{M, \infty}[G] \longleftrightarrow U_{q, \varphi}^{M, \infty}(\mathfrak{h})$, which is a monomorphism of topological $k(q)$-algebras. On the other hand $\xi_{M}^{\varphi}: F_{q, \varphi}^{M}[G]\left[\psi_{-\rho}^{-1}\right] \stackrel{\cong}{\longrightarrow} \mathbf{H}_{\varphi}^{M}$, with $\xi_{M}^{\varphi}\left(\psi_{-\rho}\right)=L_{-\rho}^{\varphi}$ (cf. the proof of Theorem 5.14); then $\epsilon\left(1-\psi_{-\rho}\right)=$ $\epsilon\left(\xi_{M}^{\varphi}\left(1-\psi_{-\rho}\right)\right)=\epsilon\left(1-L_{-\rho}^{\varphi}\right)=0$, hence $\left(1-\psi_{-\rho}\right) \in \operatorname{Ker}(\epsilon)=: \mathbf{E}_{\varphi}$; but then $\psi_{-\rho}^{-1}=\sum_{n=0}^{+\infty}\left(1-\psi_{-\rho}\right)^{n} \in F_{q, \varphi}^{M, \infty}[G]$, whence $F_{q, \varphi}^{M}[G]\left[\psi_{-\rho}^{-1}\right]$ canonically embeds into $F_{q, \varphi}^{M, \infty}[G]$, thus $\xi_{M}^{\varphi}\left(F_{q, \varphi}^{M, \infty}[G]\right) \supseteq \mathbf{H}_{\varphi}^{M}$ and then by continuity $\xi_{M}^{\varphi}\left(F_{q, \varphi}^{M, \infty}[G]\right)=U_{q, \varphi}^{M, \infty}(\mathfrak{h})$.

To prove the other parts of the claim, we can proceed as above; we only have to note, in addition, that $L_{-\rho}^{\varphi}=\prod_{i=1}^{n} L_{-\mu_{i}}^{\varphi}=\prod_{i=1}^{n}\left(M_{i}^{\varphi}\right)^{-1}$, hence $L_{\rho}^{\varphi}=\prod_{i=1}^{n} M_{i}^{\varphi}$, and $M_{i}^{\varphi}=\sum_{n=0}^{+\infty}\left(1-\left(M_{i}^{\varphi}\right)^{-1}\right)^{n}=\sum_{n=0}^{+\infty}\left(-d_{i}\right)_{q}^{n}$. $(q-1)^{n} \cdot\left(\begin{array}{c}\left(M_{i}^{\varphi}\right)^{-1} ; 0 \\ 1\end{array}\right)^{n}$ with $\left(\begin{array}{c}\left(M_{i}^{\varphi}\right)^{-1} ; 0 \\ 1\end{array}\right) \in \widehat{\mathbb{E}}^{\prime} ;$ but $\left(M_{i}^{\varphi}\right)^{-1}=\xi_{M}^{\varphi}\left(\psi_{-\mu_{i}}\right)$, so

${ }^{5}$ Of course by morphism of topological algebras we mean a morphism of algebras which is continuous. 
$\left(\begin{array}{c}\left(M_{i}^{\varphi}\right)^{-1} ; 0 \\ 1\end{array}\right)=\xi_{M}^{\varphi}\left(\left(\begin{array}{c}\psi_{-\mu_{i}} ; 0 \\ 1\end{array}\right)\right)$, with $\left(\begin{array}{c}\psi_{-\mu_{i}} ; 0 \\ 1\end{array}\right) \in \mathcal{E} ;$ then $\psi_{-\rho}^{-1}=\prod_{i=1}^{n} \psi_{-\mu_{i}}^{-1}=$ $\prod_{i=1}^{n} \sum_{n=0}^{+\infty}\left(-d_{i}\right)_{q}^{n} \cdot(q-1)^{n} \cdot\left(\begin{array}{c}\psi_{-\mu_{i}} ; 0 \\ 1\end{array}\right)^{n} \in \mathcal{F}_{\varphi}^{M, \infty}[G]$ and we conclude like for (a).

Theorem 8.7. The topological Hopf algebra $\mathfrak{F}_{\varphi}^{M, \infty}[G]$ specializes to $F^{\infty}\left[G_{M}^{\tau}\right]=F^{\infty}\left[G^{\tau}\right]$ as topological Poisson Hopf algebra for $q \rightarrow 1$, that is

$$
\begin{aligned}
\mathfrak{F}_{1, \varphi}^{M, \infty}[G] & :=\mathfrak{F}_{\varphi}^{M, \infty}[G] /(q-1) \mathfrak{F}_{\varphi}^{M, \infty}[G] \cong F^{\infty}\left[G_{M}^{\tau}\right] \\
& \cong \mathcal{U}_{\varphi}^{M, \infty}(\mathfrak{h}) /(q-1) \mathcal{U}_{\varphi}^{M, \infty}(\mathfrak{h})=: \mathfrak{U}_{1, \varphi}^{M, \infty}(\mathfrak{h}) .
\end{aligned}
$$

Proof. Recall that $F^{\infty}\left[G_{M}^{\tau}\right]$ is the $\mathfrak{e}$-adic completion of $F\left[G_{M}^{\tau}\right]$. But $\mathfrak{F}_{\varphi}^{M, \infty}[G]$ is by definition the $\mathfrak{E}_{\varphi}$-adic completion of $\mathfrak{F}_{\varphi}^{M}[G]$; since $\mathfrak{F}_{\varphi}^{M}[G] \stackrel{q \rightarrow 1}{\longrightarrow} F\left[G_{M}^{\tau}\right]$ as Poisson Hopf algebra (cf. (4.6)), $\mathfrak{F}_{\varphi}^{M, \infty}[G]$ does specialize - for $q \rightarrow 1$ — to the $\mathfrak{E}_{1}^{\tau}$-adic completion of $F\left[G_{M}^{\tau}\right]$, with $\mathfrak{E}_{1}^{\tau}:=\left.\mathfrak{E}_{\varphi}\right|_{q=1} ;$ but $\mathfrak{E}_{1}^{\tau}=\mathfrak{e}$, whence the thesis.

Remark 8.8. So far we found two topological Hopf algebras, that is $\mathfrak{F}_{\varphi}^{M, \infty}[G]$ $=\mathcal{U}_{\varphi}^{M, \infty}(\mathfrak{h})$ and $\mathcal{U}_{\varphi}^{M}(\mathfrak{h})=\mathfrak{U}_{\varphi}^{M^{\prime}}(\mathfrak{g})^{*}$, which both contain $\mathfrak{F}_{\varphi}^{M}[G]$ and for $q \rightarrow 1$ do specialize to the same object, namely $\left.\mathcal{U}_{\varphi}^{M, \infty}(\mathfrak{h})\right|_{q=1}=\left.\mathfrak{F}_{\varphi}^{M, \infty}[G]\right|_{q=1} \cong$ $\left.\mathfrak{U}_{\varphi}^{M^{\prime}}(\mathfrak{g})^{*}\right|_{q=1}=\left.\mathcal{U}_{\varphi}^{M}(\mathfrak{h})\right|_{q=1}$.

Now, next theorem shows that this is "singular fact", i. e. for "general $q$ " we have

$$
\mathcal{U}_{\varphi}^{M, \infty}(\mathfrak{h})=\mathfrak{F}_{\varphi}^{M, \infty}[G] ¥ \mathfrak{U}_{\varphi}^{M^{\prime}}(\mathfrak{g})^{*}=\mathcal{U}_{\varphi}^{M}(\mathfrak{h}) .
$$

Theorem 8.9. There does not exist any isomorphism of topological Hopf $k(q)$-algebras among $U_{q, \varphi}^{M, \infty}(\mathfrak{h})=F_{q, \varphi}^{M, \infty}[G]$ and $U_{q, \varphi}^{M^{\prime}}(\mathfrak{g})^{*}=U_{q, \varphi}^{M}(\mathfrak{h})$ whose restriction to $F_{q, \varphi}^{M}[G]$ is the identity. Hence similar statements hold for the integer forms too.

Proof. The second part of the claim follows from the first because of Proposition 8.4. Let now $\Theta: U_{q, \varphi}^{M, \infty}(\mathfrak{h})=F_{q, \varphi}^{M, \infty}[G] \stackrel{\cong}{\longrightarrow} U_{q, \varphi}^{M^{\prime}}(\mathfrak{g})^{*}=U_{q, \varphi}^{M}(\mathfrak{h})$ be an isomorphism of the above type; then $\Theta\left(L_{-\mu}^{\varphi}\right)=L_{-\mu}^{\varphi}$ for all $\mu \in M_{+}$. Let $\left\{a_{n}\right\}_{n \in \mathbb{N}} \subseteq k(q)$ be any sequence in $k(q)$; since $\left(L_{-\mu_{i}}^{\varphi}-1\right)=\left(\left(M_{i}^{\varphi}\right)^{-1}-\right.$ 1) $\in \mathbb{E}_{\varphi}^{\prime}$, we have $\sum_{n=0}^{+\infty} a_{n}\left(\left(M_{i}^{\varphi}\right)^{-1}-1\right)^{n} \in F_{q, \varphi}^{M, \infty}[G]$; therefore continuity implies $\Theta\left(\sum_{n=0}^{+\infty} a_{n}\left(\left(M_{i}^{\varphi}\right)^{-1}-1\right)^{n}\right)=\sum_{n=0}^{+\infty} \Theta\left(a_{n}\left(\left(M_{i}^{\varphi}\right)^{-1}-1\right)^{n}\right)=$ 
$\sum_{n=0}^{+\infty} a_{n}\left(\left(M_{i}^{\varphi}\right)^{-1}-1\right)^{n}$; the last should belong to $U_{q, \varphi}^{M^{\prime}}(\mathfrak{g})^{*}$, i.e., it should be a linear functional on $U_{q, \varphi}^{M^{\prime}}(\mathfrak{g})$ : but on $\Lambda_{i}^{-1}:=L_{-\nu_{i}}$ its value would be

$$
\begin{aligned}
\left\langle\sum_{n=0}^{+\infty} a_{n}\left(\left(M_{i}^{\varphi}\right)^{-1}-1\right)^{n}, \Lambda_{i}^{-1}\right\rangle_{\bar{\pi}} & =\sum_{n=0}^{+\infty} a_{n}\left\langle\left(\left(M_{i}^{\varphi}\right)^{-1}-1\right)^{n}, \Lambda_{i}^{-1}\right\rangle_{\bar{\pi}} \\
& =\sum_{n=0}^{+\infty} a_{n}\left(q_{i}-1\right)^{n}
\end{aligned}
$$

and for general $\left\{a_{n}\right\}_{n \in \mathbb{N}}$ the right-hand side is not an element of $k(q)$, contradiction.

Thus $\mathcal{U}_{\varphi}^{M, \infty}(\mathfrak{h})=\mathfrak{F}_{\varphi}^{M, \infty}[G]$ is a quantization of $F^{\infty}\left[G^{\tau}\right]$ different from $\mathcal{U}_{\varphi}^{M}(\mathfrak{h})=\mathfrak{U}_{\varphi}^{M^{\prime}}(\mathfrak{g})^{*} ;$ so also $\mathfrak{U}_{\varphi}^{M, \infty}(\mathfrak{h})=\mathcal{F}_{\varphi}^{M, \infty}[G]$ is another quantization of $U\left(\mathfrak{h}^{\tau}\right)$, different from $\mathfrak{U}_{\varphi}^{M}(\mathfrak{h})$ :

Theorem 8.10. For $q \rightarrow 1$ the topological Hopf algebra $\mathfrak{U}_{\varphi}^{M, \infty}(\mathfrak{h})=\mathcal{F}_{\varphi}^{M, \infty}[G]$ does specialize to $U\left(\mathfrak{h}^{\tau}\right)$ as a Poisson Hopf coalgebra, that is

$$
\begin{aligned}
\mathcal{F}_{1, \varphi}^{M, \infty}[G] & :=\mathcal{F}_{\varphi}^{M, \infty}[G] /(q-1) \mathcal{F}^{M, \infty}[G] \cong U\left(\mathfrak{h}^{\tau}\right) \\
& \cong \mathfrak{U}_{\varphi}^{M, \infty}(\mathfrak{h}) /(q-1) \mathfrak{U}_{\varphi}^{M, \infty}(\mathfrak{h})=: \mathfrak{U}_{1, \varphi}^{M, \infty}(\mathfrak{h})
\end{aligned}
$$

Proof. From definitions follows $\mathcal{F}_{1, \varphi}^{M, \infty}[G]=\mathcal{F}_{1, \varphi}^{M}[G]$ as Poisson Hopf coalgebras; but for Theorem 7.3 is $\mathcal{F}_{1, \varphi}^{M}[G] \cong U\left(\mathfrak{h}^{\tau}\right)$ (as Poisson Hopf coalgebras), whence the claim.

We finish with a quantum Frobenius morphism. Let $\varepsilon$ and $\ell$ be as in $\S 4.3$, and set

$\mathfrak{F}_{\varepsilon, \varphi}^{M, \infty}[G]:=\mathfrak{F}_{\varphi}^{M, \infty}[G] /(q-\varepsilon) \mathfrak{F}_{\varphi}^{M, \infty}[G] \cong \mathcal{U}_{\varphi}^{M, \infty}(\mathfrak{h}) /(q-\varepsilon) \mathcal{U}_{\varphi}^{M, \infty}(\mathfrak{h})=: \mathcal{U}_{\varepsilon, \varphi}^{M, \infty}(\mathfrak{h})$

Theorem 8.11. There exists a unique monomorphism of topological Hopf algebras

$$
\mathfrak{F r}_{G^{\tau}}^{\infty}: F^{\infty}\left[G_{M}^{\tau}\right] \cong \mathcal{U}_{1, \varphi}^{M, \infty}(\mathfrak{h})=\mathfrak{F}_{1, \varphi}^{M, \infty}[G] \longrightarrow \mathfrak{F}_{\varepsilon, \varphi}^{M, \infty}[G]=\mathcal{U}_{\varepsilon, \varphi}^{M, \infty}(\mathfrak{h})
$$

which extends $\mathfrak{F r}_{G^{\tau}}: F\left[G_{M}^{\tau}\right] \cong \mathfrak{F}_{1, \varphi}^{M}[G] \longleftrightarrow \mathfrak{F}_{\varepsilon, \varphi}^{M}[G]$ (cf. (4.7)): it is defined by

$\mathcal{F} r_{\mathfrak{g}^{\tau}}^{\infty}:\left.\left.\quad \bar{F}_{\alpha}^{\varphi}\right|_{q=1} \mapsto\left(\bar{F}_{\alpha}^{\varphi}\right)^{\ell}\right|_{q=\varepsilon},\left.\left.\quad L_{\mu}^{\varphi}\right|_{q=1} \mapsto\left(L_{\mu}^{\varphi}\right)^{\ell}\right|_{q=\varepsilon},\left.\left.\quad \bar{E}_{\alpha}^{\varphi}\right|_{q=1} \mapsto\left(\bar{E}_{\alpha}^{\varphi}\right)^{\ell}\right|_{q=\varepsilon} ;$ its image $F_{0}^{\varphi, \infty}$ is the topological Hopf subalgebra of $\mathcal{U}_{\varepsilon, \varphi}^{M, \infty}(\mathfrak{h})$ topologically generated by $\left\{\left(\bar{F}_{\alpha}^{\varphi}\right)^{\ell},\left(L_{\mu}^{\varphi}\right)^{\ell},\left(\bar{E}_{\alpha}^{\varphi}\right)^{\ell} \mid \alpha \in R^{+}, \mu \in M\right\}$, and it is contained in the centre of $\mathfrak{F}_{\varepsilon, \varphi}^{M, \infty}[G]$. 
Proof. Since $\mathfrak{F r}_{G^{\tau}}: F\left[G_{M}^{\tau}\right] \cong \mathfrak{F}_{1, \varphi}^{M}[G] \longleftrightarrow \mathfrak{F}_{\varepsilon, \varphi}^{M}[G]$ is a Hopf algebra monomorphism we have $\mathfrak{F r}_{G^{\tau}}\left(\left.\mathfrak{E}_{\varphi}\right|_{q=1}\right)=\left.\mathfrak{E}_{\varphi}\right|_{q=\varepsilon}$; but then $\mathfrak{F r}_{G^{\tau}}$ extends uniquely by continuity to a topological Hopf algebra monomorphism $\mathfrak{F}_{1, \varphi}^{M, \infty}[G] \longleftrightarrow$ $\mathfrak{F}_{\varepsilon, \varphi}^{M, \infty}[G]$ that we call $\mathfrak{F r}_{G^{\tau}}^{\infty}$. Now both $\mathfrak{F r}_{G^{\tau}}^{\infty}$ and $\mathcal{F} r_{\mathfrak{h}^{\tau}}$ are continuous extensions of $\mathfrak{F r}_{G^{\tau}}$, hence they coincide on $\mathfrak{F}_{1, \varphi}^{M}[G]$; in particular $\mathcal{F} r_{\mathfrak{h}^{\tau}}\left(\psi_{-\rho}\right)=$ $\mathfrak{F r}_{G^{\tau}}\left(\psi_{-\rho}\right)$, so that $\mathcal{F} r_{\mathfrak{h}^{\tau}}\left(\psi_{-\rho}^{-1}\right)=\mathfrak{F r}_{G^{\tau}}\left(\psi_{-\rho}^{-1}\right)$; therefore $\mathfrak{F r}_{G^{\tau}}^{\infty}$ and $\mathcal{F} \boldsymbol{r}_{\mathfrak{h}^{\tau}}$ coincide on $\mathfrak{F}_{1, \varphi}^{M}[G]\left[\psi_{-\rho}^{-1}\right]=\mathcal{H}_{\varphi}^{M}$, thus from Theorem 7.9 we get the formulas above for $\mathfrak{F r}_{G^{\tau}}^{\infty}$ : These uniquely determine it because the elements $\left.\bar{F}_{\alpha}^{\varphi}\right|_{q=1},\left.L_{\mu}^{\varphi}\right|_{q=1},\left.\bar{E}_{\alpha}^{\varphi}\right|_{q=1}$ are topological generators of $\mathcal{U}_{1, \varphi}^{M, \infty}(\mathfrak{h})=\mathfrak{F}_{1, \varphi}^{M, \infty}[G]$. Then the description of $\mathfrak{F r}_{G^{\tau}}^{\infty}\left(\mathfrak{F}_{1, \varphi}^{M, \infty}[G]\right)=F_{0}^{\varphi, \infty}$ is obvious, while the fact that $F_{0}^{\varphi, \infty}$ is contained in the centre of $\mathfrak{F}_{\varepsilon, \varphi}^{M, \infty}[G]=\mathcal{U}_{\varepsilon, \varphi}^{M, \infty}(\mathfrak{h})$ easily follows either from Theorem 7.9(b) or from [CV-2], $§ 3.3$.

\section{Appendix: The case $G=S L(2, k)$.}

For $G=S L(2, k)$ the algebra $U_{q}^{P}(\mathfrak{h})$, resp. $U_{q}^{Q}(\mathfrak{h})$, is generated by $F, L^{ \pm 1}$, resp. $K^{ \pm 1}=L^{ \pm 2}, E$. The formal Hopf algebra structure is given by

$$
\begin{gathered}
\epsilon(F)=0, \quad \epsilon\left(L^{ \pm 1}\right)=1, \quad \epsilon\left(K^{ \pm 1}\right)=1, \quad \epsilon(E)=0 \\
\Delta(F)=F \otimes 1+\sum_{n=0}^{\infty} q^{-n}\left(q-q^{-1}\right)^{2 n} \cdot K E^{n} \otimes F^{n+1} \\
\Delta(L)=\sum_{n=0}^{\infty}\left(q-q^{-1}\right)^{2 n} \cdot L E^{n} \otimes F^{n} L \\
\Delta\left(L^{-1}\right)=L^{-1} \otimes L^{-1}-\left(q-q^{-1}\right)^{2} \cdot L^{-1} E \otimes F L^{-1} \\
\Delta(K)=\sum_{n=0}^{\infty}\left(q-q^{-1}\right)^{2 n} \cdot K E^{n} \otimes F^{n} K \\
\Delta\left(K^{-1}\right)=K^{-1} \otimes K^{-1}-\left(q-q^{-1}\right)^{2} \cdot[2]_{q} \cdot K^{-1} E \otimes F K^{-1} \\
+\left(q-q^{-1}\right)^{4} \cdot K^{-1} E^{2} \otimes F^{2} K^{-1} \\
\Delta(E)=1 \otimes E+\sum_{n=0}^{\infty} q^{+n}\left(q-q^{-1}\right)^{2 n} \cdot E^{n+1} \otimes F^{n} K \\
S(F)=-q^{-2} \cdot \sum_{n=0}^{\infty}\left(q-q^{-1}\right)^{2 n} \cdot F^{n+1} K^{-(n+1)} E^{n} \\
S(L)=\sum_{n=0}^{\infty}\left(q-q^{-1}\right)^{2 n} \cdot F^{n} K^{-(n+1)} E^{n} \\
S\left(L^{-1}\right)=L-\left(q-q^{-1}\right)^{2} F L^{-1} E
\end{gathered}
$$




$$
\begin{gathered}
S(K)=\sum_{n=0}^{\infty}\left(q-q^{-1}\right)^{2 n} \cdot F^{n} K^{-(n+1)} E^{n} \\
S\left(K^{-1}\right)=K-[2]_{q} \cdot\left(q-q^{-1}\right)^{2} \cdot F E+\left(q-q^{-1}\right)^{4} \cdot F^{2} K^{-1} E^{2} \\
S(E)=-q^{+2} \cdot \sum_{n=0}^{\infty}\left(q-q^{-1}\right)^{2 n} \cdot F^{n} K^{-(n+1)} E^{n+1} .
\end{gathered}
$$

In particular from this one can prove directly all the specialization results of $\S 7$.

The quantum function algebra $F_{q}^{P}[G]=F_{q}^{P}[S L(2, k)]$ is known (cf. $[\mathbf{A P W}],[\mathbf{S V}])$ to be generated by elements $a, b, c, d$ with relations

$$
\begin{aligned}
& a b=q b a, \quad c d=q d c, \quad a c=q c a, \quad b d=q d b \\
& b c=c b, \quad a d-d a=\left(q-q^{-1}\right) b c, \quad a d-q b c=1
\end{aligned}
$$

with Hopf algebra structure defined by formulas

$$
\begin{gathered}
\Delta(a)=a \otimes a+b \otimes c, \quad \Delta(b)=a \otimes b+b \otimes d \\
\Delta(c)=c \otimes a+d \otimes c, \quad \Delta(d)=c \otimes b+d \otimes d \\
S(a)=d, \quad S(b)=-q b, \quad S(c)=-q^{-1} c, \quad S(d)=a \\
\epsilon(a)=1, \quad \epsilon(b)=0, \quad \epsilon(c)=0, \quad \epsilon(d)=1
\end{gathered}
$$

moreover $\mathfrak{F}^{P}[G]$ is nothing but the $k\left[q, q^{-1}\right]$-subalgebra of $F_{q}^{P}[G]$ generated by $a, b, c, d$.

The embedding $\xi_{P}: F_{q}^{P}[G] \longleftrightarrow U_{q}^{P}(\mathfrak{h})$ is described by formulas

$$
\begin{aligned}
\xi_{P}: \quad a \mapsto L-\left(q-q^{-1}\right)^{2} F L^{-1} E, \quad b & \mapsto-\left(q-q^{-1}\right) F L^{-1}, \\
& c \mapsto\left(q-q^{-1}\right) L^{-1} E, \quad d \mapsto L^{-1} ;
\end{aligned}
$$

then one can check directly that this is a morphism of formal Hopf algebras.

\section{References}

[APW] H.H. Andersen, P. Polo and Wen Kexin, Representations of quantum algebras, Invent. Math., 104 (1991), 1-59.

[CV-1] M. Costantini and M. Varagnolo, Quantum double and multiparameter quantum group, Comm. in Alg., 22 (1994), 6305-6321.

[CV-2] _ Multiparameter quantum function algebra at roots of 1, Math. Ann., 306 (1996), 759-780.

[DD] I. Damiani and C. De Concini, Quantum groups and Poisson groups, in W. Baldoni, M. Picardello (eds.), 'Representations of Lie groups and quantum groups', Longman Scientific and Technical, Harlow, 1994. 
[DKP] C. De Concini, V.G. Kac and C. Procesi, Quantum coadjoint action, Jour. Am. Math. Soc., 5 (1992), 151-189.

[DL] C. De Concini and V. Lyubashenko, Quantum function algebra at roots of 1 , Adv. Math., 108 (1994), 205-262.

[DP] C. De Concini and C. Procesi, Quantum groups, in L. Boutet de Monvel, C. De Concini, C. Procesi, P. Schapira, M. Vergne (eds.), 'D-modules, Representation Theory, and Quantum Groups', Lectures Notes in Mathematics, 1565, SpringerVerlag, Berlin-Heidelberg-New York, 1993.

[Di] J. Dieudonné, Introduction to the theory of formal groups, Pure and Applied Mathematics, 20, 1973.

[Dr] V.G. Drinfel'd, Quantum groups, Proc. ICM Berkeley, 1 (1986), 789-820.

[EK-1] P. Etingof and D. Kazhdan, Quantization of Lie bialgebras, I, Selecta Mathematica, New Series, 2 (1996), 1-41.

[EK-2] _ Quantization of Poisson algebraic groups and Poisson homogeneous spaces, Preprint q-alg/9510020, (1995).

[Ga] F. Gavarini, Quantum function agebras as quantum enveloping algebras, Comm. Algebra, 26 (1998), 1795-1818.

[LS] S.Z. Levendorskii and Ya.S. Soibelman, Algebras of functions on compact quantum groups, Schubert cells and quantum tori, Comm. Math. Phys., 139 (1991), 141-170.

[Lu] G. Lusztig, Quantum groups at roots of 1, Geom. Dedicata, 35 (1990), 89-113.

[Pa] P. Papi, A characterization of a good ordering in a root system, Proc. Am. Math. Soc., 120(3) (1994), 661-665.

[Re] N. Reshetikin, Multiparameter quantum groups and twisted quasitriangular Hopf algebras, Lett. Math. Phys., 20 (1990), 331-335.

[So] Ya.S. Soibelman, The algebra of functions on a compact quantum group and its representations, Leningrad Math. J., 2 (1991), 161-178.

[SV] Ya.S. Soibelman and L.L. Vaksman, Algebra of functions on the quantum group $S U(2)$, Functional Anal. Appl., 22 (1988), 170-181.

[Sw] M.E. Sweedler, Hopf algebras, Mathematics Lecture Note Series, W.A. Benjamin, Inc., New York, 1969.

Received February 10, 1997 and revised April 1, 1997.

Universitá degli Studi di Roma "Tor Vergata"

Dipartimento di MATEMATiCA

Via della Ricerca Scientifica

I-00133 Roma

ITALY

E-mail address: gavarini@mat.uniroma2.it 